\title{
A Tractable Class of Binary VCSPs via M-Convex Intersection
}

\author{
HIROSHI HIRAI and YUNI IWAMASA, University of Tokyo, Japan \\ KAZUO MUROTA, Tokyo Metropolitan University, Japan \\ STANISLAV ŽIVNÝ, University of Oxford, United Kingdom
}

\begin{abstract}
A binary VCSP is a general framework for the minimization problem of a function represented as the sum of unary and binary cost functions. An important line of VCSP research is to investigate what functions can be solved in polynomial time. Cooper-Živný classified the tractability of binary VCSP instances according to the concept of "triangle," and showed that the only interesting tractable case is the one induced by the joint winner property (JWP). Recently, Iwamasa-Murota-Živný made a link between VCSP and discrete convex analysis, showing that a function satisfying the JWP can be transformed into a function represented as the sum of two quadratic M-convex functions, which can be minimized in polynomial time via an M-convex intersection algorithm if the value oracle of each $\mathrm{M}$-convex function is given.

In this paper, we give an algorithmic answer to a natural question: What binary finite-valued CSP instances can be represented as the sum of two quadratic M-convex functions and can be solved in polynomial time via an M-convex intersection algorithm? We solve this problem by devising a polynomial-time algorithm for obtaining a concrete form of the representation in the representable case. Our result presents a larger tractable class of binary finite-valued CSPs, which properly contains the JWP class.
\end{abstract}

\section{CCS Concepts: • Theory of computation $\rightarrow$ Design and analysis of algorithms;}

Additional Key Words and Phrases: Valued constraint satisfaction problems, discrete convex analysis, Mconvexity

\section{ACM Reference Format:}

Hiroshi Hirai, Yuni Iwamasa, Kazuo Murota, and Stanislav Živný. 2018. A Tractable Class of Binary VCSPs via MConvex Intersection. ACM Trans. Algor. V, N, Article A (January 2018), 40 pages. https://doi.org/0000001.0000001

\section{INTRODUCTION}

The valued constraint satisfaction problem (VCSP) provides a general framework for discrete optimization (see [39] for details). Informally, the VCSP framework deals with the minimization problem of a function represented as the sum of "small" arity functions, which are called cost functions. It is known that various kinds of combinatorial optimization problems can be formulated in the VCSP framework. In general, the VCSP is NP-hard. An important line of research is to investigate what restrictions on classes of VCSP instances ensure polynomial time solvability. Two main types of VCSPs with restrictions are structure-based VCSPs and language-based VCSPs (see e.g., [24]).

A preliminary version of this paper [17] has appeared in the proceedings of the 35th International Symposium on Theoretical Aspects of Computer Science (STACS 2018).

Authors' addresses: Hiroshi Hirai; Yuni Iwamasa, University of Tokyo, Department of Mathematical Informatics, Graduate School of Information Science and Technology, Tokyo, 113-8656, Japan; Kazuo Murota, Tokyo Metropolitan University, Department of Business Administration, Tokyo, 192-0397, Japan; Stanislav Živný, University of Oxford, Department of Computer Science, Oxford, OX1 3QD, United Kingdom.

Permission to make digital or hard copies of all or part of this work for personal or classroom use is granted without fee provided that copies are not made or distributed for profit or commercial advantage and that copies bear this notice and the full citation on the first page. Copyrights for components of this work owned by others than the author(s) must be honored Abstracting with credit is permitted. To copy otherwise, or republish, to post on servers or to redistribute to lists, requires prior specific permission and/or a fee. Request permissions from permissions@acm.org.

(C) 2018 Copyright held by the owner/author(s). Publication rights licensed to the Association for Computing Machinery. 1549-6325/2018/January-ARTA \$15.00

https://doi.org/0000001.0000001 
Structure-based VCSPs deal with restrictions on graph structures representing the appearance of variables in a given instance. For example, it is known (e.g., [1]) that if the graph (named the Gaifman graph) corresponding to a VCSP instance has a bounded treewidth then the instance can be solved in polynomial time. Language-based VCSPs deal with restrictions on cost functions that appear in a VCSP instance. Kolmogorov-Thapper-Živný [22] gave a precise characterization of tractable valued constraint languages via the basic LP relaxation. Kolmogorov-Krokhin-Rolínek [21] gave a dichotomy for all language-based VCSPs (see also $[3,38]$ for a dichotomy for all language-based CSPs).

Hybrid VCSPs, which deal with a combination of structure-based and language-based restrictions, have emerged recently [7]. Among many kinds of hybrid restrictions, a binary VCSP, VCSP with only unary and binary cost functions, is a representative hybrid restriction that includes numerous fundamental optimization problems. Cooper-Živný [5] showed that if a given binary VCSP instance satisfies the joint winner property ( $(F W P)$, then it can be minimized in polynomial time. The same authors classified in [6] the tractability of binary VCSP instances according to the concept of "triangle," and showed that the only interesting tractable case is the one induced by the JWP (see also [7]). Furthermore, they introduced cross-free convexity as a generalization of JWP, and devised a polynomial-time minimization algorithm for cross-free convex instances $F$ when a "cross-free representation" of $F$ is given; see related works below for details.

In this paper, we introduce a novel tractability principle going beyond triangle and cross-free representation for binary finite-valued CSPs, denoted from now on as binary VCSPs. A binary VCSP is formulated as follows, where $D_{1}, D_{2}, \ldots, D_{r}(r \geq 2)$ are finite sets.

Given: Unary cost functions $F_{p}: D_{p} \rightarrow \mathrm{R}$ for $p \in\{1,2, \ldots, r\}$ and binary cost functions $F_{p q}: D_{p} \times D_{q} \rightarrow \mathrm{R}$ for $1 \leq p<q \leq r$.

Problem: Find a minimizer of $F: D_{1} \times D_{2} \times \cdots \times D_{r} \rightarrow \mathbf{R}$ defined by

$$
F\left(X_{1}, X_{2}, \ldots, X_{r}\right):=\sum_{1 \leq p \leq r} F_{p}\left(X_{p}\right)+\sum_{1 \leq p<q \leq r} F_{p q}\left(X_{p}, X_{q}\right) .
$$

Our tractability principle is built on discrete convex analysis $(D C A)[28,30]$, which is a theory of convex functions on discrete structures. In DCA, L-convexity and M-convexity play primary roles; the former is a generalization of submodularity, and the latter is a generalization of matroids. A variety of polynomially solvable problems in discrete optimization can be understood within the framework of L-convexity/M-convexity (see e.g., [30-32]). Recently, it has also turned out that discrete convexity is deeply linked to tractable classes of VCSPs. L-convexity is closely related to the tractability of language-based VCSPs. Various kinds of submodularity induce tractable classes of language-based VCSP instances [22], and a larger class of such submodularity can be understood as L-convexity on certain graph structures [14]; see also [15]. On the other hand, Iwamasa-MurotaŽivný [20] have pointed out that M-convexity plays a role in hybrid VCSPs. They revealed the reason for the tractability of a VCSP instance satisfying the JWP from a viewpoint of M-convexity. We here continue this line of research, and explore further applications of M-convexity in hybrid VCSPs.

A function $f:\{0,1\}^{n} \rightarrow \mathbf{R} \cup\{+\infty\}$ is called $M$-convex $[25,30]$ if it satisfies the following generalization of the matroid exchange axiom: for $x=\left(x_{1}, x_{2}, \ldots, x_{n}\right)$ and $y=\left(y_{1}, y_{2}, \ldots, y_{n}\right)$ with $f(x)<+\infty$ and $f(y)<+\infty$, and $i \in\{1,2, \ldots, n\}$ with $x_{i}>y_{i}$, there exists $j \in\{1,2, \ldots, n\}$ with $y_{j}>x_{j}$ such that

$$
f(x)+f(y) \geq f\left(x-\chi_{i}+\chi_{j}\right)+f\left(y+\chi_{i}-\chi_{j}\right),
$$

where $\chi_{i}$ is the $i$ th unit vector. Although $\mathrm{M}$-convex functions are defined on $\mathbf{Z}^{n}$ in general, we only need functions on $\{0,1\}^{n}$ here. M-convex functions on $\{0,1\}^{n}$ are equivalent to the negative of 
valuated matroids introduced by Dress-Wenzel $[10,11]$. An M-convex function can be minimized in a greedy fashion similarly to the greedy algorithm for matroids. Furthermore, a function $f$ : $\{0,1\}^{n} \rightarrow \mathbf{R} \cup\{+\infty\}$ that is representable as the sum of two M-convex functions is called $M_{2}$-convex. In particular, $f$ is called quadratically representable $M_{2}$-convex $\left(Q R-M_{2}\right.$-convex) if $f$ is representable as the sum of two quadratic M-convex functions. As a generalization of matroid intersection, the problem of minimizing an $\mathrm{M}_{2}$-convex function, called the $M$-convex intersection problem, can also be solved in polynomial time if the value oracle of each constituent M-convex function is given [26, 27]; see also [29, Section 5.2]. Our proposed tractable class of VCSPs is based on this result.

Let us return to binary VCSPs. The starting observation for relating VCSP to DCA is that the objective function $F$ on $D_{1} \times D_{2} \times \cdots \times D_{r}$ can be regarded as a function $f$ on $\{0,1\}^{n}$ with $n:=\sum_{1 \leq p \leq r}\left|D_{p}\right|$ by the following correspondence between the domains:

$$
D_{p}:=\left\{1,2, \ldots, n_{p}\right\} \ni i \longleftrightarrow(\underbrace{0, \ldots, 0, \stackrel{i}{1}, 0, \ldots, 0}_{n_{p}})
$$

With this correspondence, the minimization of $F$ can be transformed to that of $f$. A binary VCSP instance $F$ is said to be quadratic $M_{2}$-representable if the function $f$ obtained from $F$ via the correspondence (1.2) is $\mathrm{QR}-\mathrm{M}_{2}$-convex.

It is shown in [20] that a binary VCSP instance satisfying the JWP can be transformed to a quadratic $\mathrm{M}_{2}$-representable instance, $\mathbb{1}$ and two $\mathrm{M}$-convex summands can be obtained in polynomial time. Here the following natural question arises: What binary VCSP instances are quadratic $M_{2}-$ representable? In this paper, we give an algorithmic answer to this question by considering the following problem:

\section{Testing Quadratic $\mathrm{M}_{2}$-Representability}

Given: A binary VCSP instance $F$.

Problem: Determine whether $F$ is quadratic $\mathrm{M}_{2}$-representable or not. If $F$ is quadratic $\mathrm{M}_{2}$ representable, obtain a decomposition $f=f_{1}+f_{2}$ of the function $f$ into two quadratic M-convex functions $f_{1}$ and $f_{2}$, where $f$ is the function transformed from $F$ via (1.2).

Our main result is the following:

Theorem 1.1. Testing Quadratic $M_{2}$-Representability can be solved in $O\left(n^{4}\right)$ time.

An $\mathrm{M}_{2}$-convex function $f$ can be minimized in polynomial time if such a decomposition can be obtained in polynomial time. Thus we obtain the following corollary of Theorem 1.1.

COROLlARY 1.2. A quadratic $M_{2}$-representable binary VCSP instance can be minimized in polynomial time.

Overview. We outline our approach to Testing QuAdRATIC $M_{2}$-Representability via taking a small concrete example of a quadratic $\mathrm{M}_{2}$-representable binary VCSP instance. Suppose that $D_{1}=D_{2}=D_{3}=D_{4}=\{0,1\}$. Unary cost functions $F_{1}, F_{2}, F_{3}, F_{4}$ and binary cost functions $F_{p q}$

$\mathbb{I}$ In [20], a binary VCSP instance satisfying the JWP was transformed into the sum of two quadratic $\mathrm{M}^{\natural}$-convex functions. It can be easily seen that this function can also be transformed into the sum of two quadratic M-convex functions. 
$(1 \leq p<q \leq 4)$ are given by

$$
\begin{array}{rlrl}
F_{1}:=\left[\begin{array}{l}
1 \\
0
\end{array}\right], & F_{2}:=\left[\begin{array}{l}
0 \\
1
\end{array}\right], \quad F_{3}:=\left[\begin{array}{l}
1 \\
0
\end{array}\right], \quad F_{4}:=\left[\begin{array}{l}
0 \\
1
\end{array}\right], \\
F_{12}:=\left[\begin{array}{ll}
3 & 0 \\
1 & 4
\end{array}\right], & F_{13}:=\left[\begin{array}{ll}
2 & 0 \\
1 & 3
\end{array}\right], & F_{14}:=\left[\begin{array}{ll}
1 & 0 \\
0 & 1
\end{array}\right], \\
F_{23}:=\left[\begin{array}{ll}
2 & 0 \\
0 & 2
\end{array}\right], & F_{24}:=\left[\begin{array}{ll}
3 & 0 \\
1 & 1
\end{array}\right], & F_{34}:=\left[\begin{array}{ll}
2 & 0 \\
0 & 0
\end{array}\right],
\end{array}
$$

where $F_{p q}$ is regarded as a $2 \times 2$ matrix with the $(i, j)$-component $F_{p q}(i-1, j-1)$ for $1 \leq i, j \leq 2$, and $F_{p}$ is also regarded as a 2-dimensional vector in a similar way. Based on the correspondence (1.2), the function $f$ on $\{0,1\}^{8}$ is constructed as follows (this construction will be introduced formally in Section 2.1):

$$
f(x):=\frac{1}{2} x^{\top}\left[\begin{array}{cc|c|c|c}
0 & \infty & F_{12} & F_{13} & F_{14} \\
\infty & 0 & & \\
\hline F_{12}^{\top} & 0 & \infty & F_{23} & F_{24} \\
\hline F_{13}^{\top} & F_{23}^{\top} & 0 & \infty & F_{34} \\
\hline F_{14}^{\top} & F_{24}^{\top} & F_{34}^{\top} & 0 & \infty \\
\hline
\end{array}\right] x+\left[\begin{array}{c}
F_{1} \\
\hline F_{2} \\
\hline F_{3} \\
\hline F_{4}
\end{array}\right]^{\top} x
$$

for $x \in\{0,1\}^{8}$ with $\sum_{1 \leq i \leq 8} x_{i}=4$ and $f(x):=+\infty$ for other $x$. Recall that $F$ is quadratic $\mathrm{M}_{2}$ representable if and only if $f$ is QR- $\mathrm{M}_{2}$-convex and that $F$ is efficiently minimizable if and only if so is $f$.

Our algorithm constructs the following two M-convex summands $f_{1}$ and $f_{2}$ of $f$ :

$$
f_{1}(x):=\frac{1}{2}\left[\begin{array}{c}
x_{1} \\
x_{3} \\
x_{5} \\
x_{7} \\
x_{4} \\
x_{8} \\
x_{2} \\
x_{6}
\end{array}\right]^{\top}\left[\begin{array}{ll|l|l|lll}
6 & 6 & 4 & 2 & & & \\
6 & 6 & 4 & 2 & & & \\
\hline 4 & 4 & 4 & 2 & & & \\
\hline 2 & 2 & 2 & 2 & & & \\
\hline & & & 2 & 2 \\
& & & & & & \\
& & & & & \\
& & & & & \\
& & & & &
\end{array}\right]\left[\begin{array}{c}
x_{1} \\
x_{3} \\
x_{5} \\
x_{7} \\
x_{4} \\
x_{8} \\
x_{2} \\
x_{6}
\end{array}\right]
$$

and

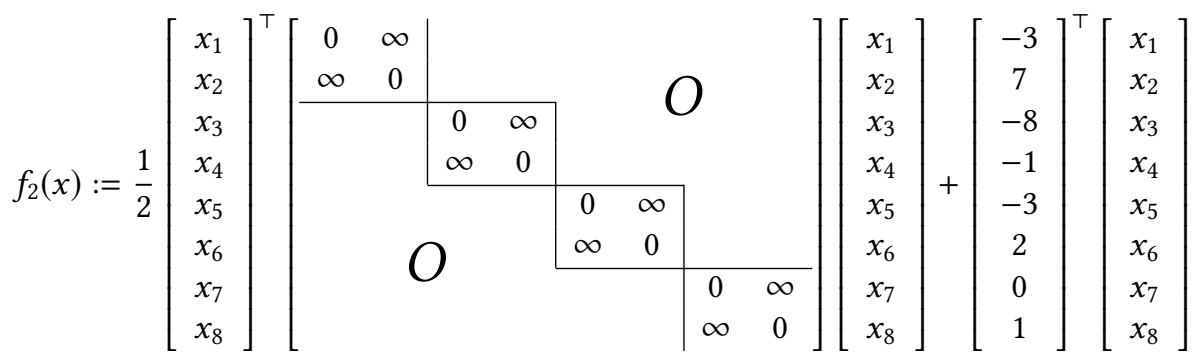

for $x \in\{0,1\}^{8}$ with $\sum_{1 \leq i \leq 8} x_{i}=4$, and $f_{1}(x):=+\infty$ and $f_{2}(x):=+\infty$ for other $x$. The first function $f_{1}$ in (1.5) is a laminar convex function [30, Section 6.3], which is a typical example of M-convex 
functions. Indeed, by using a laminar family $\mathcal{L}=\{\{1,3,5,7\},\{1,3,5\},\{1,3\},\{4,8\}\}, f_{1}$ is written as

$$
f_{1}(x)=\sum_{X \in \mathcal{L}}\left(\sum_{i \in X} x_{i}\right)^{2} .
$$

The second function $f_{2}$ in (1.6) is nothing but a linear function on the base family of the partition matroid with partition $\{\{1,2\},\{3,4\},\{5,6\},\{7,8\}\}$, and hence $f_{2}$ is also M-convex.

We establish a representation theorem (Theorem 2.3), which says that $\mathrm{QR}-\mathrm{M}_{2}$-convex functions arising from binary VCSP instances always admit the above type of the decomposition. For a set $X \subseteq\{1,2, \ldots, n\}$, let $\bar{\ell}_{X}$ be the quadratic function defined on $\{0,1\}^{n}$ by

$$
\bar{\ell}_{X}(x):=\left(\sum_{i \in X} x_{i}\right)^{2} .
$$

The theorem states that a function $f$ arising from a binary VCSP instance is $\mathrm{QR}-\mathrm{M}_{2}$-convex if and only if $f$ is a laminar convex function restricted to the base family of the partition matroid with partition $\mathcal{A}$ of $\{1,2, \ldots, n\}$, i.e.,

$$
f=\sum_{X \in \mathcal{L}} c_{X} \bar{\ell}_{X}+h+\delta_{\mathcal{A}}
$$

where $\mathcal{L}$ is a laminar family, $c_{X}$ is a positive weight on $X \in \mathcal{L}, h$ is a linear function, and $\delta_{\mathcal{A}}$ is the $\{0,+\infty\}$-function taking 0 on the bases and $+\infty$ on the non-bases.

The main difficulty in solving Testing QUADRATIC $M_{2}$-REPRESENTABILITy is that a representation of quadratic functions on the base family of the partition matroid is not unique. Indeed, we see that the coefficients in (1.4) do not equal the sum of coefficients in (1.5) and (1.6). In particular, $\bar{\ell}_{X}$ satisfies the following relations:

$$
\begin{aligned}
& \bar{\ell}_{X}+\delta_{\mathcal{A}}=\bar{\ell}_{X \cup A_{p}}+h+\delta_{\mathcal{A}} \quad \text { if } A_{p} \in \mathcal{A} \text { and } A_{p} \cap X=\emptyset, \\
& \bar{\ell}_{X}+\delta_{\mathcal{A}}=\bar{\ell}_{\{1,2, \ldots, n\} \backslash X}+h^{\prime}+\delta_{\mathcal{A}},
\end{aligned}
$$

where $h$ and $h^{\prime}$ are linear functions. This means that $f$ can be QR-M ${ }_{2}$-convex even if $f$ is written as

$$
f=\sum_{X \in \mathcal{F}} c_{X} \bar{\ell}_{X}+h+\delta_{\mathcal{A}}
$$

for a non-laminar family $\mathcal{F}$. Based on this consideration, we divide Testing QUAdRAtic $\mathrm{M}_{2}$ REPRESENTABILITy into two subproblems named DeComposition and LAMINARIZATION.

Decomposition is the problem of obtaining a representation (1.11) of a given QR-M $\mathrm{M}_{2}$-convex function $f$ for some family $\mathcal{F}$ not necessarily laminar but laminarizable by repeating the following transformations corresponding to (1.9) and (1.10):

$$
\begin{aligned}
& X \mapsto X \cup A_{p} \text { or } X \backslash A_{p} \\
& X \mapsto\{1,2, \ldots, n\} \backslash X
\end{aligned}
$$

for $X \in \mathcal{F}$, where $A_{p} \cap X=\emptyset$ or $A_{p} \subseteq X$. We present a polynomial-time algorithm for DECOMPOSITION in Section 3. LAMINARIZATION is the problem of constructing a laminar family $\mathcal{L}$ from the family $\mathcal{F}$ obtained in Decomposition by repeating the transformations (1.12) and (1.13). LAMINARIZATION can be seen as a purely combinatorial problem for a set system. We present a polynomial-time algorithm for LAMINARIZATION in Section 4.

If we apply our Decomposition algorithm to $f$ in (1.4), we obtain a representation (1.11) for a nonlaminar family $\mathcal{F}:=\{\{1,3,5,7\},\{1,3,5\},\{2,4\},\{3,7\}\}$, where $\mathcal{A}:=\{\{1,2\},\{3,4\},\{5,6\},\{7,8\}\}$. 

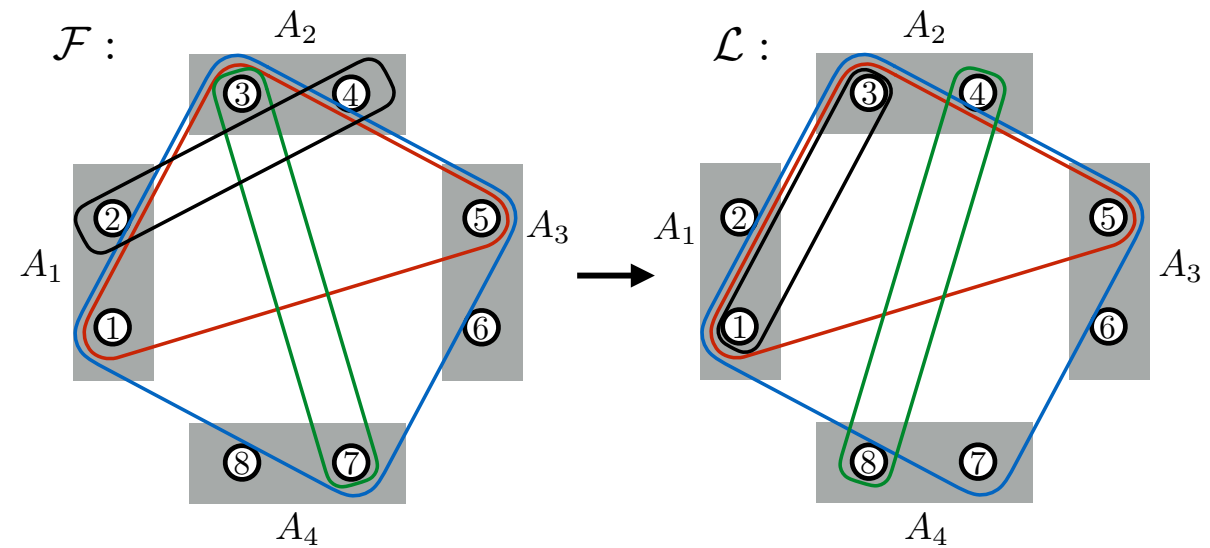

Fig. 1. The left figure illustrates the input $\mathcal{F}$ of LAMINARIzATION and the right figure illustrates an output laminar family $\mathcal{L}$, where black nodes indicate elements of $\{1,2, \ldots, 8\}$, gray rectangles indicate members in $\mathcal{A}$, and solid curves indicate four sets in $\mathcal{F}$ and $\mathcal{L}$, respectively.

Then, by solving LaminaRization for $\mathcal{F}$, we obtain a laminar family $\mathcal{L}:=\{\{1,3,5,7\},\{1,3,5\},\{1,3\},\{4,8\}\}$. Indeed, we can transform $\{2,4\}$ to $\{1,3\}$ by repeating transformations (1.12) and (1.13) since $\{1,3\}=(\{1,2, \ldots, 8\} \backslash\{2,4\}) \backslash\{5,6,7,8\}$. See Figure 1 . Thus we can verify the $\mathrm{QR}-\mathrm{M}_{2}$-convexity of $f$ by constructing two M-convex summands of $f$.

Application to quadratic pseudo-Boolean function minimization. Pseudo-Boolean function minimization is a fundamental and well-studied problem in theoretical computer science (see e.g., $[2,8]$ ). Our result provides a new tractable class of quadratic pseudo-Boolean functions minimization. Consider a pseudo-Boolean function $F:\{0,1\}^{n} \rightarrow \mathrm{R}$ represented as

$$
F\left(x_{1}, x_{2}, \ldots, x_{n}\right)=\sum_{1 \leq i<j \leq n} a_{i j} x_{i} x_{j}+\sum_{1 \leq i \leq n} a_{i} x_{i} .
$$

Then $F$ is lifted to $f:\{0,1\}^{2 n} \rightarrow \mathbf{R} \cup\{+\infty\}$ defined by: For $x \in\{0,1\}^{2 n}$ with $\sum_{1 \leq i \leq 2 n} x_{i}=n$,

$$
f\left(x_{1}, \ldots, x_{n}, x_{n+1}, \ldots, x_{2 n}\right):=\sum_{1 \leq i<j \leq n} a_{i j} x_{i} x_{j}+\sum_{1 \leq i \leq n} \infty \cdot x_{i} x_{n+i}+\sum_{1 \leq i \leq n} a_{i} x_{i},
$$

and for other $x, f(x):=+\infty$. Then $F\left(x_{1}, \ldots, x_{n}\right)=f\left(x_{1}, \ldots, x_{n}, 1-x_{1}, \ldots, 1-x_{n}\right)$ for any $x \in\{0,1\}^{n}$. Hence minimizing $F$ is equivalent to minimizing $f$.

We can regard $f$ as a function arising from the binary VCSP instance $F$ with the partition $\mathcal{A}:=\left\{A_{1}, A_{2}, \ldots, A_{n}\right\}$ of $\{1,2, \ldots, 2 n\}$ given by $A_{i}=\{i, n+i\}$ for $i=1,2, \ldots, n$. Therefore, if $f$ is QR-M $\mathrm{M}_{2}$-convex, then we can obtain two M-convex functions $f_{1}$ and $f_{2}$ satisfying $f=f_{1}+f_{2}$ by our proposed algorithm, and we can minimize $f$ (and hence $F$ ) in polynomial time.

As far as we are aware, all tractable classes of (exactly minimizable) pseudo-Boolean functions are related to (i) bounded treewidth, (ii) submodularity, or (iii) a switching reduction (which flips the values of a subset of the variables) to (ii) $[2,8]$. To the best of our knowledge, our new tractable class is incomparable with the existing one $[2,8]$. We are not aware of any nontrivial known tractable class contained in ours.

\section{Related works.}

- Cooper-Živný [5] introduced the joint winner property (JWP) for binary VCSP instances as a sufficient condition for tractability. A binary VCSP instance $F$ of the form (1.1) is said to 
satisfy the $\mathcal{F W P}$ if

$$
F_{i j}(a, b) \geq \min \left\{F_{i k}(a, c), F_{j k}(b, c)\right\}
$$

for all distinct $i, j, k \in[r]$ and all $a \in D_{i}, b \in D_{j}, c \in D_{k}$. It is shown in [5] that if $F$ satisfies the JWP, then $F$ can be transformed, in polynomial time, into a function $F^{\prime}$ satisfying the JWP, $\operatorname{argmin} F^{\prime} \subseteq \operatorname{argmin} F$, and the additional special property named the $Z$-freeness, and that $Z$-free instances can be minimized in polynomial time. Thus, if $F$ satisfies the JWP, then $F$ can be minimized in polynomial time. Furthermore, Iwamasa-Murota-Živný [20] revealed that $\mathrm{Z}$-free instances are quadratic $\mathrm{M}_{2}$-representable.

The tractability based on quadratic $\mathrm{M}_{2}$-representability depends solely on the function values, and is independent of how the function $F$ is given. Indeed, a quadratic $\mathrm{M}_{2}$-representable instance $F$ can be characterized by the existence of a Z-free instance $F^{\prime}$ that satisfies $F^{\prime}(X)=$ $F(X)$ for all $X$. This stands in sharp contrast with the tractability based on the JWP, which depends heavily on the representation of $F$. For example, let $F(X)=\sum F_{p}\left(X_{p}\right)+\sum F_{p q}\left(X_{p}, X_{q}\right)$ be a binary VCSP instance satisfying the JWP. By choosing a pair of distinct $p, q \in\{1,2, \ldots, r\}$, $d \in D_{p}$, and $\alpha \in \mathbf{R}$ arbitrarily, replace $F_{p}(d)$ and $F_{p q}\left(d, X_{q}\right)$ by $F_{p}(d)+\alpha$ and $F_{p q}\left(d, X_{q}\right)-\alpha$, respectively. Then $F$ does not change but violates the JWP in general. For example, although the binary VCSP instance $F$ in (1.3) does not satisfy the JWP by $F_{12}(1,1)=4, F_{13}(1,0)=1$, and $F_{23}(1,0)=0, F$ is quadratic $\mathrm{M}_{2}$-representable. Thus our result can explore such hidden $\mathrm{M}_{2}$-convexity.

- Cooper-Živný [6] introduced a generalization of JWP, named cross-free convexity, for not necessarily binary VCSP instances. A VCSP instance $F: D_{1} \times D_{2} \times \cdots \times D_{r} \rightarrow \mathbf{R} \cup\{+\infty\}$ is said to be cross-free convex if the function $f:\{0,1\}^{n} \rightarrow \mathbf{R} \cup\{+\infty\}$ obtained from $F$ via correspondence (1.2) can be represented as

$$
f(x)=\sum_{X \in \mathcal{F}} g_{X}\left(\sum_{i \in X} x_{i}\right),
$$

where $\mathcal{F} \subseteq 2^{[n]}$ is cross-free and, for each $X \in \mathcal{F}, g_{X}$ is a univariate function on $Z$ satisfying $g_{X}(m-1)+g_{X}(m+1) \geq 2 g_{X}(m)$ for all $m \in \mathbf{Z}$. Here the equality (1.14) is required for every $x \in\{0,1\}^{n}$ that corresponds to some $X \in D_{1} \times D_{2} \times \cdots \times D_{r}$ via (1.2) and $f(x)=+\infty$ for other $x$. A pair $X, Y \subseteq[n]$ is said to be crossing if $X \cap Y,[n] \backslash(X \cup Y), X \backslash Y$, and $Y \backslash X$ are all nonempty, and a family $\mathcal{F} \subseteq 2^{[n]}$ is said to be cross-free if there is no crossing pair in $\mathcal{F}$.

Cross-free convexity is a special class of $\mathrm{M}_{2}$-representability, where a VCSP instance $F$ is $M_{2}$-representable if the function $f$ obtained from $F$ via correspondence (1.2) is $\mathrm{M}_{2}$-convex. Indeed, it follows from a similar argument to Lemma 2.1 below that $f$ in (1.14) is $\mathrm{M}_{2}$-convex. Hence, a cross-free convex instance $F$ is $\mathrm{M}_{2}$-representable.

Our result provides, for binary finite-valued CSPs, a polynomial-time minimization algorithm for special cross-free convex instances (quadratic $\mathrm{M}_{2}$-representable instances) even when the expression (1.14) is not given.

- Our representation theorem (Theorem 2.3) is inspired by the polyhedral split decomposition due to Hirai [13]. This general decomposition principle decomposes, by means of polyhedral geometry, a function on a finite set $\mathcal{D}$ of points of $\mathbf{R}^{n}$ into a sum of simpler functions, called split functions, and a residue term. This aspect can be explained for our function $f$ in (1.4) roughly as follows. The expression $\sum_{X \in \mathcal{L}} c_{X} \bar{\ell}_{X}+f_{2}$ of $f$ can be viewed as the polyhedral split decomposition of $f$, where $\mathcal{D}$ is equal to the effective domain of $f, c_{X} \bar{\ell}_{X}$ on $\mathcal{D}$ is a sum of split functions and a linear function (cf. (2.2)) for each $X \in \mathcal{L}$, and $f_{2}$ defined by (1.6) is a residue term. 
- Interestingly, LAMINARIZATION appears in a completely different problem in computational biology. A phylogenetic tree is a graphical representation of an evolutionary history in a set of taxa in which the leaves correspond to taxa and the non-leaves correspond to speciations. One of the important problems in phylogenetic analysis is to assemble a global phylogenetic tree from smaller pieces of phylogenetic trees, particularly, quartet trees. QUARTET COMPATIBILITY is to decide whether there is a phylogenetic tree inducing a given collection of quartet trees, and to construct such a phylogenetic tree if it exists. It is known [36] that QUARTET Compatibility is NP-hard.

As a subsequent work to the present paper, Hirai-Iwamasa [16] have introduced two novel classes of quartet systems, named complete multipartitite quartet systems and full multipartite quartet systems, and showed that QUARTET COMPATIBILITy for these quartet systems can be solved in polynomial time. In their algorithms, the algorithm proposed in this paper for LAMINARIZATION is utilized for polynomial-time solvability.

Notation. Let $\mathbf{Z}, \mathbf{R}, \mathbf{R}_{+}$, and $\mathbf{R}_{++}$denote the sets of integers, reals, nonnegative reals, and positive reals, respectively. In this paper, functions can take the infinite value $+\infty$, where $a<+\infty, a+\infty=$ $+\infty$ for $a \in \mathbf{R}$, and $0 \cdot(+\infty)=0$. Let $\overline{\mathbf{R}}:=\mathbf{R} \cup\{+\infty\}$. For a function $f:\{0,1\}^{n} \rightarrow \mathbf{R} \cup\{+\infty\}$, the effective domain is denoted as $\operatorname{dom} f:=\left\{x \in\{0,1\}^{n} \mid f(x)<+\infty\right\}$. For a positive integer $k$, we define $[k]:=\{1,2, \ldots, k\}$. We often abbreviate a set $\left\{i_{1}, i_{2}, \ldots, i_{k}\right\}$ as $i_{1} i_{2} \cdots i_{k}$.

\section{REPRESENTATION OF QR-M $\mathbf{M}_{2}$-CONVEX FUNCTIONS}

For a partition $\mathcal{A}:=\left\{A_{1}, A_{2}, \ldots, A_{r}\right\}$ of $[n]$, let $\delta_{\mathcal{A}}:\{0,1\}^{n} \rightarrow \overline{\mathbf{R}}$ be the indicator function of the base family of a partition matroid with partition $\mathcal{A}$, that is, $\delta_{\mathcal{A}}(x):=0$ if $\sum_{i \in A_{p}} x_{i}=1$ for each $p \in[r]$ and $\delta_{\mathcal{A}}(x):=+\infty$ otherwise. Let $U_{\mathcal{A}}$ be the set of characteristic vectors of the bases of a partition matroid with partition $\mathcal{A}$, i.e., $U_{\mathcal{A}}:=\left\{x \in\{0,1\}^{n} \mid \sum_{i \in A_{p}} x_{i}=1(p \in[r])\right\}=\operatorname{dom} \delta_{\mathcal{A}}$. Let $U_{n, r}$ be the set of characteristic vectors of the bases of the uniform matroid on $[n]$ of rank $r$, i.e., $U_{n, r}:=\left\{x \in\{0,1\}^{n} \mid \sum_{i \in[n]} x_{i}=r\right\}$. Note that $U_{\mathcal{A}} \subsetneq U_{n, r}$.

\subsection{Representation theorem}

We introduce a class of quadratic functions on $\{0,1\}^{n}$ that has a bijective correspondence to binary VCSP instances. Let $\mathcal{A}:=\left\{A_{1}, A_{2}, \ldots, A_{r}\right\}$ be a partition of $[n]$ with $\left|A_{p}\right| \geq 2$ for $p \in[r]$. We say that $f:\{0,1\}^{n} \rightarrow \overline{\mathbf{R}}$ is a VCSP-quadratic function of type $\mathcal{A}$ if $f$ is represented as

$$
f(x):= \begin{cases}\sum_{1 \leq i<j \leq n} a_{i j} x_{i} x_{j}+\sum_{1 \leq i \leq n} a_{i} x_{i} & \text { if } x \in U_{n, r}, \\ +\infty & \text { otherwise }\end{cases}
$$

for some $a_{i} \in \mathbf{R}$ and $a_{i j} \in \overline{\mathbf{R}}$ such that $a_{i j}=+\infty$ for $i, j \in A_{p}(p \in[r])$ and $a_{i j}<+\infty$ for $i \in A_{p}$ and $j \in A_{q}(p, q \in[r], p \neq q)$. We assume $a_{i j}=a_{j i}$ for distinct $i, j \in[n]$; see (1.4).

Suppose that a binary VCSP instance $F$ of the form (1.1) is given, where we assume $F_{p q}=F_{q p}$ for distinct $p, q \in[r]$. The transformation of $F$ to $f$ based on (1.2) in Section 1 is formalized as follows. Choose a partition $\mathcal{A}:=\left\{A_{1}, A_{2}, \ldots, A_{r}\right\}$ of $[n]$ with $\left|A_{p}\right|=n_{p}\left(=\left|D_{p}\right|\right)$ and identify $A_{p}$ with $D_{p}$. Define

$$
\begin{aligned}
a_{i} & :=F_{p}(i) \quad(i \in[n]), \\
a_{i j} & := \begin{cases}F_{p q}(i, j) & \left(i \in A_{p} \text { and } j \in A_{q} \text { for some distinct } p, q \in[r]\right), \\
+\infty & \left(i, j \in A_{p} \text { for some } p \in[r]\right) .\end{cases}
\end{aligned}
$$

Then the function $f$ in (2.1) is a VCSP-quadratic function of type $\mathcal{A}$. 
We introduce two functions that will serve as the M-convex summands of an $\mathrm{M}_{2}$-convex VCSPquadratic function of type $\mathcal{A}$. A function $h:\{0,1\}^{n} \rightarrow \overline{\mathbf{R}}$ is said to be $\mathcal{A}$-linear if $h$ is a linear function on $U_{\mathcal{A}}$, that is, if $h$ can be represented as $h(x)=\delta_{\mathcal{A}}(x)+\sum_{1 \leq i \leq n} u_{i} x_{i}+\gamma$ for some linear coefficient $\left(u_{i}\right)_{i \in[n]}$ and constant $\gamma \in \mathbf{R}$. We use such $h$ as an M-convex summand. As the other function, for technical reasons, we use the following $\ell_{X}$ instead of $\bar{\ell}_{X}$ in (1.8); the difference of $\bar{\ell}_{X}$ and $2 \ell_{X}$ is linear. For $X \subseteq[n]$, let $\ell_{X}:\{0,1\}^{n} \rightarrow \mathbf{R}$ be defined by

$$
\ell_{X}(x):=\sum_{i, j \in X, i<j} x_{i} x_{j}
$$

The following lemma guarantees the M-convexity of the two functions (like $f_{1}$ in (1.5) and $f_{2}$ in (1.6)) obtained in our algorithm. Here a family $\mathcal{F} \subseteq 2^{[n]}$ is said to be laminar if $X \subseteq Y, X \supseteq Y$, or $X \cap Y=\emptyset$ holds for all $X, Y \in \mathcal{F}$.

LEMма 2.1. (1) An $\mathcal{A}$-linear function is M-convex.

(2) For any laminar family $\mathcal{L}$ and positive weight $c$ on $\mathcal{L}$, the function $\sum_{X \in \mathcal{L}} c(X) \ell_{X}$ on $U_{n, r}$ is M-convex.

Proof. (1). An $\mathcal{A}$-linear function $h$ can be viewed as a linear function on the base family of a partition matroid with partition $\mathcal{A}$. Hence $h$ is M-convex.

(2). We can see that the quadratic coefficient of $\sum_{X \in \mathcal{L}} c(X) \ell_{X}$ satisfies $a_{i j}+a_{k l} \geq \min \left\{a_{i k}+\right.$ $\left.a_{j l}, a_{i l}+a_{j k}\right\}$ for every distinct $i, j, k, l \in[n]$ (see also Lemma 2.5 below). Hence, by [19, Theorem 3.1] (or Lemma 2.4 (I) below), $\sum_{X \in \mathcal{L}} c(X) \ell_{X}$ on $U_{n, r}$ is M-convex.

Lemma 2.1 gives a sufficient condition for the QR-M $\mathrm{M}_{2}$-convexity of a VCSP-quadratic function $f$; if $f$ can be represented as the sum of $\sum_{X \in \mathcal{L}} c(X) \ell_{X}$ on $U_{n, r}$ for some laminar $\mathcal{L}$ and a linear function on $U_{\mathcal{A}}$, then $f$ is QR-M $\mathrm{M}_{2}$-convex. Our representation theorem (Theorem 2.3) says that this is also a necessary condition, that is, a QR-M $\mathrm{M}_{2}$-convex VCSP-quadratic function is always representable as the sum of $\sum_{X \in \mathcal{L}} c(X) \ell_{X}$ on $U_{n, r}$ for some laminar $\mathcal{L}$ and a linear function on $U_{\mathcal{A}}$

A laminar family inducing the given $\mathrm{QR}-\mathrm{M}_{2}$-convex VCSP-quadratic function possesses some kind of uniqueness, which ensures the validity of our proposed algorithm. To describe the uniqueness in Theorem 2.3, we introduce an equivalence relation on functions:

- For two functions $f$ and $f^{\prime}$ on $\{0,1\}^{n}$, we say that $f$ and $f^{\prime}$ are $\mathcal{A}$-linear equivalent (or $f \simeq f^{\prime}$ ) if the difference between $f$ and $f^{\prime}$ is a linear function on $U_{\mathcal{A}}$, that is, $f+\delta_{\mathcal{A}}=f^{\prime}+h$ holds for some $\mathcal{A}$-linear function $h$.

The $\mathcal{A}$-linear equivalence on $\ell_{X}$ 's can be regarded as a combinatorial property on sets $X$ by using the following notations.

- We say that a set $X \subseteq[n]$ cuts $A_{p}$ if both $X$ and $[n] \backslash X$ have a nonempty intersection with $A_{p}$, i.e., $\emptyset \neq X \cap A_{p} \neq A_{p}$.

- A set $X \subseteq[n]$ is called an $\mathcal{A}$-cut if $X$ cuts at least two elements in $\mathcal{A}$.

- For $X \subseteq[n]$, the cutting support of $X$, denote by $\langle X\rangle$, is defined as the union of $A_{p}$ 's cut by $X$. That is,

$$
\langle X\rangle:=\bigcup\left\{A_{p} \in \mathcal{A} \mid \emptyset \neq X \cap A_{p} \neq A_{p}\right\} .
$$

Lемма 2.2. (1) For $X \subseteq[n], \ell_{X}+\delta_{\mathcal{A}}$ is not $\mathcal{A}$-linear if and only if $X$ is an $\mathcal{A}$-cut.

(2) For two $\mathcal{A}$-cuts $X$ and $Y$, functions $\ell_{X}$ and $\ell_{Y}$ are $\mathcal{A}$-linear equivalent if and only if

$$
\{\langle X\rangle \cap X,\langle X\rangle \backslash X\}=\{\langle Y\rangle \cap Y,\langle Y\rangle \backslash Y\},
$$

that is, $X$ and $Y$ have the same cutting support and yield the same bipartition on it. 
Proof. As in (1.8) in the introduction, define $\bar{\ell}_{X}:\{0,1\}^{n} \rightarrow \mathbf{R}$ by $\bar{\ell}_{X}(x):=\left(\sum_{i \in X} x_{i}\right)^{2}$. Then it holds $\ell_{X} \simeq \bar{\ell}_{X} / 2$ by $x_{i}^{2}=x_{i}$ for $i \in[n]$. Hence it suffices to show the statements for $\bar{\ell}_{X}$. As mentioned in (1.9) and (1.10), it holds (i) $\bar{\ell}_{X} \simeq \bar{\ell}_{X \cup A_{p}}$ if $X \cap A_{p} \neq \emptyset$, and (ii) $\bar{\ell}_{X} \simeq \bar{\ell}_{[n] \backslash X}$. The former follows from $\bar{\ell}_{X \cup A_{p}}(x)=\left(\sum_{i \in X} x_{i}+\sum_{i \in A_{p}} x_{i}\right)^{2} \simeq\left(\sum_{i \in X} x_{i}+1\right)^{2} \simeq \bar{\ell}_{X}(x)$, and the latter follows from $\bar{\ell}_{[n] \backslash X}(x) \simeq\left(r-\sum_{i \in X} x_{i}\right)^{2} \simeq \bar{\ell}_{X}(x)$.

(Only-if part of (1)). Suppose that $X$ is not an $\mathcal{A}$-cut. Then $\langle X\rangle \subseteq A_{p}$ holds for some $A_{p} \in \mathcal{A}$. By (i), we may assume $X \subseteq A_{p}$. Then it holds $\bar{\ell}_{X}(x)=\left(\sum_{i \in X} x_{i}\right)^{2}=\sum_{i \in X} x_{i}$ for all $x \in U_{\mathcal{A}}$, implying that $\bar{\ell}_{X}$ is $\mathcal{A}$-linear.

(If part of (2)). Suppose that (2.4) holds. Then we can construct $Y$ from $X$ by repeating the transformation $X \mapsto[n] \backslash X, X \cup A_{p}$, or $X \backslash A_{p}$ for $A_{p}$ with $\langle X\rangle \cap A_{p}=\emptyset$. Hence $\bar{\ell}_{X} \simeq \bar{\ell}_{Y}$ by (i) and (ii) above.

(If part of (1)). To detect the non-linearity, we consider the following four points $x^{s u}, x^{s v}, x^{t u}, x^{t v} \in$ $U_{\mathcal{A}}$ for distinct $s, t \in A_{p}$ and $u, v \in A_{q}$ with distinct $p, q \in[r]$ :

- $x_{i}^{i j}=x_{j}^{i j}=1$ for $i=s, t$ and $j=u, v$, and

- $x_{i}^{s u}=x_{i}^{s v}=x_{i}^{t u}=x_{i}^{t v}$ for $i \in[n] \backslash\left(A_{p} \cup A_{q}\right)$.

Since $x^{s u}+x^{t v}=x^{s v}+x^{t u}$, the inequality $\bar{\ell}_{X}\left(x^{s u}\right)+\bar{\ell}_{X}\left(x^{t v}\right) \neq \bar{\ell}_{X}\left(x^{s v}\right)+\bar{\ell}_{X}\left(x^{t u}\right)$ implies that $\bar{\ell}_{X}$ is not linear on the four points. Let $\kappa_{X}:=\left(\bar{\ell}_{X}\left(x^{s u}\right)+\bar{\ell}_{X}\left(x^{t v}\right)\right)-\left(\bar{\ell}_{X}\left(x^{s v}\right)+\bar{\ell}_{X}\left(x^{t u}\right)\right)$. By $\bar{\ell}_{X}\left(x^{i j}\right)=(|X \cap\{i\}|+|X \cap\{j\}|+k)^{2}$ with a constant $k$, we have

$$
\kappa_{X}= \begin{cases}2 & \text { if } X \cap\{s, t, u, v\}=\{s, u\} \text { or }\{t, v\}, \\ -2 & \text { if } X \cap\{s, t, u, v\}=\{s, v\} \text { or }\{t, u\}, \\ 0 & \text { otherwise. }\end{cases}
$$

If $X$ is an $\mathcal{A}$-cut, we can choose distinct $s, t \in A_{p}, u, v \in A_{q}$ for distinct $A_{p}, A_{q} \subseteq\langle X\rangle$ such that $|X \cap\{s, t\}|=|X \cap\{u, v\}|=1$, and it holds $\kappa_{X} \neq 0$.

(Only-if part of (2)). This can be shown in a similar way as the proof of the if part of (1). Suppose that (2.4) does not hold. Then we can choose distinct $s, t \in A_{p}, u, v \in A_{q}$ with $p \neq q$ such that $\kappa_{X} \neq \kappa_{Y}$, which implies that $\bar{\ell}_{X}$ and $\bar{\ell}_{Y}$ are not $A$-linear equivalent. Indeed, by $\{\langle X\rangle \cap X,\langle X\rangle \backslash X\} \neq$ $\{\langle Y\rangle \cap Y,\langle Y\rangle \backslash Y\}$, there are $A_{p}$ and $A_{q}$ cut by (say) $X$ such that $\left\{\left(A_{p} \cup A_{q}\right) \cap X,\left(A_{p} \cup A_{q}\right) \backslash X\right\} \neq$ $\left\{\left(A_{p} \cup A_{q}\right) \cap Y,\left(A_{p} \cup A_{q}\right) \backslash Y\right\}$. Hence we can choose points $s \in A_{p} \cap X, t \in A_{p} \backslash X, u \in A_{q} \cap X$, and $v \in A_{q} \backslash X$ such that at least one of $s \notin A_{p} \cap Y, t \notin A_{p} \backslash Y, u \notin A_{q} \cap Y$, and $v \notin A_{q} \backslash Y$ holds Then (2.5) shows $\kappa_{X} \neq \kappa_{Y}$.

According to Lemma 2.2, we introduce the equivalence relations on sets, families, and positive weights on families, and also introduce the concept of laminarizability as follows.

- For two $\mathcal{A}$-cuts $X$ and $Y$, we say that $X$ and $Y$ are $\mathcal{A}$-equivalent (or $X \sim Y$ ) if $X$ and $Y$ satisfy (2.4). That is, $X \sim Y$ if and only if $\ell_{X} \simeq \ell_{Y}$.

- The $\mathcal{A}$-equivalence relation is naturally extended to $\mathcal{A}$-cut families $\mathcal{F}, \mathcal{G}$ by: $\mathcal{F}$ and $\mathcal{G}$ are $\mathcal{A}$-equivalent (or $\mathcal{F} \sim \mathcal{G}$ ) if the set of the equivalence classes of all $\mathcal{A}$-cuts in $\mathcal{F}$ coincides with that of $G$.

- We define the $\mathcal{A}$-equivalence relation $\sim$ between a positive weight $c$ on $\mathcal{F}$ and a positive weight $d$ on $\mathcal{G}$ by: $c \sim d$ if $\mathcal{F} \sim \mathcal{G}$ and $\sum_{Y \in \mathcal{F}: Y \sim X} c(Y)=\sum_{Y \in \mathcal{G}: Y \sim X} d(Y)$ for all $X \subseteq[n]$. It is clear, by the definition of $\sim$, that if $\mathcal{F} \sim \mathcal{G}$ and $c \sim d$, then $\sum_{X \in \mathcal{F}} c(X) \ell_{X} \simeq \sum_{X \in \mathcal{G}} d(X) \ell_{X}$.

- An $\mathcal{A}$-cut family $\mathcal{F}$ is said to be laminarizable if there is a laminar family $\mathcal{L}$ with $\mathcal{F} \sim \mathcal{L}$.

The formal description of our representation theorem is the following. 
THEOREM 2.3. Let $f$ be a VCSP-quadratic function of type $\mathcal{A}=\left\{A_{1}, A_{2}, \ldots, A_{r}\right\}$. Then $f$ is $Q R-M_{2}$-convex if and only if there exist a laminarizable $\mathcal{A}$-cut family $\mathcal{F}$ and a positive weight $c$ on $\mathcal{F}$ such that

$$
f \simeq \sum_{X \in \mathcal{F}} c(X) \ell_{X}
$$

In addition, $\mathcal{F}$ and $c$ are uniquely determined up to $\sim$.

The proof of Theorem 2.3 is given in Sections 2.3 and 2.4.

\subsection{Two subproblems: DECOMPOSITION and LAMINARIZATION}

By Theorem 2.3, Testing Quadratic $\mathrm{M}_{2}$-Representability can be divided into the following two problems: (i) if $f$ is $Q R-M_{2}$-convex, then output a laminarizable $\mathcal{A}$-cut family $\mathcal{F}$ and a positive weight $c$ on $\mathcal{F}$ satisfying the equation (2.6), and (ii) if the output $\mathcal{F}$ of (i) is laminarizable, then find a laminar family $\mathcal{L}$ with $\mathcal{L} \sim \mathcal{F}$. (i) and (ii) can be formulated as Decomposition and LAMINARIZATION, respectively. An $\mathcal{A}$-cut family $\mathcal{F}$ is said to be non-redundant if no distinct $X, Y$ with $X \sim Y$ are contained in $\mathcal{F}$.

Decomposition

Given: A VCSP-quadratic function $f$ of type $\mathcal{A}$.

Problem: Either detect the non-QR- $\mathrm{M}_{2}$-convexity of $f$, or obtain some non-redundant $\mathcal{A}$-cut family $\mathcal{F}$ and positive weight $c$ on $\mathcal{F}$ satisfying

$$
f \simeq \sum_{X \in \mathcal{F}} c(X) \ell_{X}
$$

In addition, in case where $f$ is $\mathrm{QR}-\mathrm{M}_{2}$-convex, $\mathcal{F}$ is required to be laminarizable.

We emphasize that Decomposition may possibly output the decomposition (2.7) even when the input $f$ is not $\mathrm{QR}-\mathrm{M}_{2}$-convex. However, if Decomposition detects the non- $\mathrm{QR}-\mathrm{M}_{2}$-convexity then we can conclude that the input $f$ is not $\mathrm{QR}-\mathrm{M}_{2}$-convex.

\section{LAMINARIZATION}

Given: A non-redundant $\mathcal{A}$-cut family $\mathcal{F}$.

Problem: Determine whether $\mathcal{F}$ is laminarizable or not. If it is laminarizable, obtain a nonredundant laminar $\mathcal{A}$-cut family $\mathcal{L}$ with $\mathcal{F} \sim \mathcal{L}$.

With these procedures, Testing Quadratic $\mathrm{M}_{2}$-Representability is solved as follows.

- Suppose that $f$ is $\mathrm{QR}-\mathrm{M}_{2}$-convex. First, by solving Decomposition, we obtain a non-redundant laminarizable $\mathcal{A}$-cut family $\mathcal{F}$ and a positive weight $c$ on $\mathcal{F}$ satisfying (2.7) (and hence (2.6)). Then, by solving LAMINARIZATION with $\mathcal{F}$ as its input, we obtain a non-redundant laminar $\mathcal{A}$ cut family $\mathcal{L}$ with $\mathcal{L} \sim \mathcal{F}$. Thus we can obtain two M-convex summands $f_{1}:=\sum_{X \in \mathcal{L}} c^{*}(X) \ell_{X}$ on $U_{n, r}$ and $f_{2}:=f-\sum_{X \in \mathcal{L}} c^{*}(X) \ell_{X}$, where $c^{*} \sim c$. Such $c^{*}$ can easily be constructed as $c^{*}(X):=c(Y)$ for $X \in \mathcal{L}$ and $Y \in \mathcal{F}$ with $X \sim Y$.

- Suppose that $f$ is not $\mathrm{QR}-\mathrm{M}_{2}$-convex. By solving Decomposition, we can detect the non-QR$\mathrm{M}_{2}$-convexity of $f$ or we obtain some $\mathcal{A}$-cut family $\mathcal{F}$, positive weight $c$ on $\mathcal{F}$, and $\mathcal{A}$-linear function $h$ that demonstrates (2.7). In the former case, we are done. In the latter case, by solving LAMINARIZATION with $\mathcal{F}$ as its input, we can detect the non-laminarizability of $\mathcal{F}$, which denies the QR-M $\mathrm{M}_{2}$-convexity of $f$.

We devise an $O\left(r n^{2}\right)$-time algorithm for Decomposition in Section 3 and an $O\left(n^{4}\right)$-time algorithm for LAMINARIZATION in Section 4. Thus we obtain Theorem 1.1.

By Lemma 2.2 (2), LAMINARIZATION can be regarded as the problem of transforming a given family $\mathcal{F}$ to a laminar family by repeating the following operation: replace $X \in \mathcal{F}$ with $[n] \backslash X$, 
$X \cup A_{p}$, or $X \backslash A_{p}$ with some $A_{p}$ satisfying $\langle X\rangle \cap A_{p}=\emptyset$. Figure 1 illustrates an example of the input (left) and an output (right) of LAMINARIZATION.

\subsection{Proof of Theorem 2.3: Characterization}

In this subsection, we prove the if-and-only-if part of Theorem 2.3, i.e., a VCSP-quadratic function $f$ of type $\mathcal{A}$ is $Q R-M_{2}$-convex if and only if (2.6) holds for some laminarizable $\mathcal{A}$-cut family $\mathcal{F}$ and positive weight $c$ on $\mathcal{F}$.

We first review fundamental facts about a general quadratic (not necessarily VCSP-quadratic) function $g:\{0,1\}^{n} \rightarrow \overline{\mathbf{R}}$ represented as

$$
g\left(x_{1}, x_{2}, \ldots, x_{n}\right)= \begin{cases}\sum_{i, j \in[n], i<j} a_{i j} x_{i} x_{j}+\sum_{i \in[n]} a_{i} x_{i} & \text { if } x \in U_{n, r}, \\ +\infty & \text { otherwise }\end{cases}
$$

where $r \in \mathrm{Z}$ with $r \geq 2, a_{i} \in \mathbf{R}$, and $a_{i j}=a_{j i} \in \overline{\mathbf{R}}$. We assume the following regularity condition (R) for $g$.

(R): For all $i \in[n]$, there is $x=\left(x_{1}, x_{2}, \ldots, x_{n}\right)$ such that $g(x)<+\infty$ and $x_{i}=1$.

Denote the indicator function of dom $g$ by $\delta_{g}$, which is defined as $\delta_{g}(x):=0$ for $x \in \operatorname{dom} g$ and $\delta_{g}(x):=+\infty$ for $x \notin \operatorname{dom} g$.

Let $G_{g}^{\infty}$ be the graph on node set $[n]$ such that edge $\{i, j\}(i \neq j)$ exists if and only if $a_{i j}=+\infty$. Define $m\left(G_{g}^{\infty}\right)$ as the number of connected components of $G_{g}^{\infty}$. A connected component with at least one edge is said to be non-isolated. The number of non-isolated connected components of $G_{g}^{\infty}$ will be denoted by $m^{*}=m^{*}\left(G_{g}^{\infty}\right)$. Let $B_{1}, B_{2}, \ldots, B_{m^{*}}$ be the node sets of the non-isolated connected components of $G_{g}^{\infty}$.

Then the M-convexity of $g$ is characterized by the following lemma, which is a refinement of the results of [18] and [33].

LEMMA 2.4 ([19, TheOREM 3.1]). A function $g$ of the form (2.8) satisfying condition (R) is Mconvex if and only if each connected component of $G_{g}^{\infty}$ is a complete graph and one of the following conditions (I), (II), and (III) holds:

(I): $m\left(G_{g}^{\infty}\right) \geq r+2$ and

$$
a_{i j}+a_{k l} \geq \min \left\{a_{i k}+a_{j l}, a_{i l}+a_{j k}\right\}
$$

holds for every distinct $i, j, k, l \in[n]$.

(II): $m\left(G_{g}^{\infty}\right)=r+1$ and

$$
a_{i j}+a_{k l}=a_{i l}+a_{j k}
$$

holds for every $p \in\left[m^{*}\right]$, distinct $i, k \in B_{p}$, and distinct $j, l \in[n] \backslash B_{p}$.

(III): $m\left(G_{g}^{\infty}\right)=r$ and

$$
a_{i j}+a_{k l}=a_{i l}+a_{j k}
$$

holds for every distinct $p, q \in\left[m^{*}\right]$, distinct $i, k \in B_{p}$, and distinct $j, l \in B_{q}$.

Moreover, (II) or (III) holds if and only if $g$ is represented as $g(x)=\delta_{g}(x)+\sum_{i \in[n]} u_{i} x_{i}+\gamma$ for some $u \in \mathbf{R}^{n}$ and $\gamma \in \mathbf{R}$.

We say that $\left(a_{i j}\right)_{i, j \in[n]}$ satisfies the anti-tree metric property if (2.9) holds, and that $\left(a_{i j}\right)_{i, j \in[n]}$ satisfies the anti-ultrametric property if

$$
a_{i j} \geq \min \left\{a_{i k}, a_{j k}\right\}
$$


holds for all distinct $i, j, k \in[n]$. It is known [9] that the anti-ultrametric property is stronger than the anti-tree metric property (2.9). The anti-ultrametric property is related with a laminar family as in Lemma 2.5 below. A subpartition of $[n]$ is a family of disjoint subsets of $[n]$. For a subpartition $\mathcal{B}$, a family $\mathcal{L}$ is said to be $\mathcal{B}$-laminar if $\mathcal{L}$ is laminar and $B \subsetneq X$ or $B \cap X=\emptyset$ holds for each $B \in \mathcal{B}$ and $X \in \mathcal{L}$, that is, if $\mathcal{L}$ does not intersect with $\mathcal{B}, \mathcal{L} \cup \mathcal{B}$ is laminar, and each $B \in \mathcal{B}$ is minimal in $\mathcal{L} \cup \mathcal{B}$

LемMA 2.5 ([20, LемMA 8]). Let $g$ be a quadratic function with a coefficient $\left(a_{i j}\right)_{i, j \in[n]}$, and $\mathcal{B}$ be the family of the node sets of the non-isolated connected components of $G_{g}^{\infty}$. Then $\left(a_{i j}\right)_{i, j \in[n]}$ satisfies the anti-ultrametric property if and only if $a_{i j}$ can be represented as

$$
a_{i j}= \begin{cases}+\infty & \text { if } i, j \in B \text { for some } B \in \mathcal{B}, \\ \sum\{c(L) \mid L \in \mathcal{L} ; i, j \in L\}+\alpha^{*} & \text { otherwise }\end{cases}
$$

for some $\mathcal{B}$-laminar family $\mathcal{L} \subseteq 2^{[n]} \backslash[n]$ and some positive weight c on $\mathcal{L}$, where $\alpha^{*}:=\min _{i, j \in[n]} a_{i j}$.

Lemma 2.5 follows from Lemma 8 of [20] by relating $\mathcal{B}$ to the set of complete graphs for $\alpha=+\infty$ and relating $\mathcal{L}$ to the union of the set of complete graphs for $\alpha<+\infty$, where $\alpha$ is a parameter appearing in Lemma 8 of [20].

The following is a variation of a well-known technique (the Farris transform) in phylogenetics [35] to transform a tree metric to an ultrametric, and is implied by the validity of Algorithm I described in Section 4.1 of [19]. In particular, Steps 1 and 2 of Algorithm I correspond to the following.

Lемма 2.6 ([19]). Suppose that $\left(a_{i j}\right)_{i, j \in[n]}$ satisfies the anti-tree metric property. Let $\alpha^{*}:=$ $\min _{i, j \in[n]} a_{i j}$ and $b_{k}:=\min _{j \in[n]} a_{k j}-\alpha^{*}$ for $k \in[n]$. Then $\min _{j \in[n]} a_{i j}=\alpha^{*}$ holds for all $i \in[n]$, and $\left(a_{i j}-b_{i}-b_{j}\right)_{i, j \in[n]}$ satisfies the anti-ultrametric property.

We now ready to show the characterization part of Theorem 2.3. Note that, by the definition of laminarizability, (2.6) holds for some laminarizable family $\mathcal{F}$ if and only if (2.6) holds for some laminar family $\mathcal{L}$.

Proposition 2.7 (The characterization Part of Theorem 2.3). Let $f$ be a VCSPquadratic function of type $\mathcal{A}$. Then $f$ is $Q R-M_{2}$-convex if and only if

$$
f \simeq \sum_{X \in \mathcal{L}} c(X) \ell_{X}
$$

for some laminar $\mathcal{A}$-cut family $\mathcal{L}$ and positive weight c on $\mathcal{L}$.

Proof. For a subpartiton $\mathcal{B}$, define $\delta_{\mathcal{B}}:\{0,1\}^{n} \rightarrow \overline{\mathbf{R}}$ by $\delta_{\mathcal{B}}(x):=0$ if $x \in U_{n, r}$ and $\sum_{i \in B} x_{i} \leq 1$ for each $B \in \mathcal{B}$, and $\delta_{\mathcal{B}}(x):=+\infty$ otherwise. Then, by Lemma $2.4, \delta_{\mathcal{B}}$ is an M-convex function that can be represented as $\delta_{\mathcal{B}}(x)=\sum_{B \in \mathcal{B}} \sum_{i, j \in B, i<j} \infty \cdot x_{i} x_{j}$ on $U_{n, r}$. The set of non-isolated connected components of $G_{\delta_{\mathcal{B}}}^{\infty}$ is equal to $\mathcal{B}$. We say that a function $f$ is of Type I, Type II, or Type III if $m\left(G_{f}^{\infty}\right) \geq r+2, m\left(G_{f}^{\infty}\right)=r+1$, or $m\left(G_{f}^{\infty}\right)=r$ holds, respectively (c.f., Lemma 2.4).

(If part). By the $\mathcal{A}$-linear equivalence, $f$ is represented as $f=\sum_{X \in \mathcal{L}} c(X) \ell_{X}+h$ for some $\mathcal{A}$ linear function $h$. By Lemma 2.1 (1) and (2), the functions $h$ and $\sum_{X \in \mathcal{L}} c(X) \ell_{X}$ on $U_{n, r}$ are M-convex. Hence $f$ is QR-M $\mathrm{M}_{2}$-convex.

(Only-if part). Let $f_{1}, f_{2}:\{0,1\}^{n} \rightarrow \overline{\mathbf{R}}$ be any quadratic M-convex functions with $f=f_{1}+f_{2}$. Since $f$ satisfies condition (R), $f_{1}$ and $f_{2}$ also satisfy condition (R) by $\operatorname{dom} f=\operatorname{dom} f_{1} \cap \operatorname{dom} f_{2}$. Let $\mathcal{B}_{1}$ and $\mathcal{B}_{2}$ be the sets of non-isolated connected components of $G_{f_{1}}^{\infty}$ and $G_{f_{2}}^{\infty}$, respectively. Since $f_{1}$ and $f_{2}$ are M-convex, each member of $\mathcal{B}_{1}$ (resp. $\mathcal{B}_{2}$ ) induces a complete graph in $G_{f_{1}}^{\infty}$ (resp. $G_{f_{2}}^{\infty}$ ) by Lemma 2.4. Hence $\operatorname{dom} f_{1}=\operatorname{dom} \delta_{\mathcal{B}_{1}}$ and $\operatorname{dom} f_{2}=\operatorname{dom} \delta_{\mathcal{B}_{2}}$ hold. Note that $\operatorname{dom} f=\operatorname{dom} \delta_{\mathcal{A}}=$ $\operatorname{dom}\left(\delta_{\mathcal{B}_{1}}+\delta_{\mathcal{B}_{2}}\right)$. 
Here the following claim holds.

CLAIm. There exist quadratic $M$-convex functions $f_{1}$ and $f_{2}$ such that $f=f_{1}+f_{2}, \mathcal{B}_{1} \cap \mathcal{B}_{2}=\emptyset$, and $\mathcal{B}_{1} \cup \mathcal{B}_{2}=\mathcal{A}$.

Proof of Claim. Let $f_{1}, f_{2}:\{0,1\}^{n} \rightarrow \overline{\mathbf{R}}$ be any quadratic M-convex functions with $f=f_{1}+f_{2}$. We first show that if $\mathcal{B}_{1}$ and $\mathcal{B}_{2}$ satisfy (i) for each $B \in \mathcal{B}_{1} \cup \mathcal{B}_{2}$ there is $A \in \mathcal{A}$ such that $B \subseteq A$, and (ii) each $A \in \mathcal{A}$ belongs to $\mathcal{B}_{1} \cup \mathcal{B}_{2}$ (i.e., $\mathcal{A} \subseteq \mathcal{B}_{1} \cup \mathcal{B}_{2}$ ), then Claim holds.

Suppose that (i) and (ii) hold, and that some $B \in \mathcal{B}_{1}$ violates $\mathcal{B}_{1} \cap \mathcal{B}_{2}=\emptyset$ or $\mathcal{B}_{1} \cup \mathcal{B}_{2}=\mathcal{A}$, i.e., $B \in \mathcal{B}_{2}$ or $B \notin \mathcal{A}$. Then we can modify $f_{1}$ so that $f_{1}$ is M-convex with $f=f_{1}+f_{2}$ and $\operatorname{dom} f_{1}$ is changed from dom $\delta_{\mathcal{B}_{1}}$ to $\operatorname{dom} \delta_{\mathcal{B}_{1} \backslash\{B\}}$ as follows.

By (i) and (ii), there is $A \in \mathcal{A} \cap \mathcal{B}_{2}$ such that $B \subseteq A$. If $f_{1}$ is of Type II or III, then $f_{1} \simeq \delta_{\mathcal{B}_{1}}$ by Lemma 2.4. Hence we have

$$
f_{1}+f_{2} \simeq \delta_{\mathcal{B}_{1}}+f_{2}=\delta_{\mathcal{B}_{1} \backslash\{B\}}+f_{2},
$$

where the second equality follows from $\operatorname{dom}\left(\delta_{\mathcal{B}_{1}}+f_{2}\right)=\operatorname{dom}\left(\delta_{\mathcal{B}_{1} \backslash\{B\}}+f_{2}\right)$ by $B \subseteq A$ and $A \in \mathcal{B}_{2}$. Thus we can modify $f_{1}$ so that $f_{1}$ is M-convex with $f=f_{1}+f_{2}$ and $\operatorname{dom} f_{1}=\operatorname{dom} \delta_{\mathcal{B}_{1} \backslash\{B\}}$. If $f_{1}$ is of Type I, then, by Lemma 2.4 (I) and Lemma 2.6, the quadratic coefficient of $f_{1}$ are represented as $\left(a_{i j}^{1}+b_{i}+b_{j}\right)_{i, j \in[n]}$, where $b_{i} \in \mathbf{R}$ and $\left(a_{i j}^{1}\right)$ satisfies the anti-ultrametric property. By modifying $a_{i j}^{1}(=+\infty)$ to $M$ for $i, j \in B_{1}$ with a sufficiently large $M$, we have $\operatorname{dom} f_{1}=\operatorname{dom} \delta_{\mathcal{B}_{1} \backslash\left\{B_{1}\right\}}$ and the value of $f_{1}+f_{2}$ does not change. Furthermore $\left(a_{i j}^{1}\right)$ still satisfies the anti-ultrametric property. Hence $f_{1}$ is M-convex. Thus we can modify $f_{1}$ so that $f_{1}$ is M-convex with $f=f_{1}+f_{2}$ and $\operatorname{dom} f_{1}=\operatorname{dom} \delta_{\mathcal{B}_{1} \backslash\{B\}}$. By repeating the above modification for $f_{1}$ or $f_{2}$, we obtain the $f_{1}$ and $f_{2}$ in Claim.

We finally show that (i) and (ii) hold.

(i). We can easily see that, for every $i, j$ with $a_{i j}<+\infty$ (i.e., $i \in A_{p}$ and $j \in A_{q}$ for some distinct $p, q$ ), there is $x \in \operatorname{dom} f$ such that $x_{i}=x_{j}=1$. Hence, for such $i, j$, there is no $B \in \mathcal{B}_{1} \cup \mathcal{B}_{2}$ satisfying $i, j \in B$. Therefore we obtain (i).

(ii). Let $E_{\mathcal{A}}$ and $E_{\mathcal{B}}$ be the edge set of $G_{\delta_{\mathcal{A}}}^{\infty}$ and of $G_{\mathcal{B}_{1}+\delta_{\mathcal{B}_{2}}}^{\infty}$, respectively. That is, $\{i, j\} \in E_{\mathcal{A}}$ (resp. $\{i, j\} \in E_{\mathcal{B}}$ ) if and only if $i, j \in A$ for some $A \in \mathcal{A}$ (resp. $i, j \in B$ for some $B \in \mathcal{B}_{1} \cup \mathcal{B}_{2}$ ). By (i), we have $E_{\mathcal{A}} \supseteq E_{\mathcal{B}}$. Suppose, to the contrary, that $E_{\mathcal{A}} \supsetneq E_{\mathcal{B}}$. Then there is $\{i, j\}$ such that $i, j \in A_{p}$ for some $p$ and $\{i, j\} \notin E_{\mathcal{B}}$. Let $x \in\{0,1\}^{n}$ be a 0 - 1 vector such that $x_{i}=x_{j}=1, \sum_{i \in[n]} x_{i}=r$, and $\sum_{i \in A_{q}} x_{i} \leq 1$ for each $q$ distinct from $p$. Since $E_{\mathcal{A}} \supseteq E_{\mathcal{B}}$, we have $x \in \operatorname{dom}\left(\delta_{\mathcal{B}_{1}}+\delta_{\mathcal{B}_{2}}\right)$, whereas $x \notin \operatorname{dom} \delta_{\mathcal{A}}$. This contradicts $\operatorname{dom} \delta_{\mathcal{A}}=\operatorname{dom}\left(\delta_{\mathcal{B}_{1}}+\delta_{\mathcal{B}_{2}}\right)$, and hence $E_{\mathcal{A}}=E_{\mathcal{B}}$ holds. Therefore we obtain (ii).

This completes the proof of Claim.

By Claim, we can take quadratic M-convex functions $f_{1}$ and $f_{2}$ satisfying $f=f_{1}+f_{2}$, dom $f_{1}=$ $\operatorname{dom} \delta_{\mathcal{B}_{1}}$, and $\operatorname{dom} f_{2}=\operatorname{dom} \delta_{\mathcal{B}_{2}}$, where $\mathcal{B}_{1} \cap \mathcal{B}_{2}=\emptyset$ and $\mathcal{B}_{1} \cup \mathcal{B}_{2}=\mathcal{A}$. In the following, we show that $f=f_{1}+f_{2}$ satisfies (2.14) with some laminar $\mathcal{A}$-cut family $\mathcal{L}$ and positive weight $c$ on $\mathcal{L}$ for each of the three cases: (i) both $f_{1}$ and $f_{2}$ are of Type II or III, (ii) $f_{1}$ is of Type I and $f_{2}$ is of Type II or III, and (iii) both $f_{1}$ and $f_{2}$ are of Type I.

(i). By Lemma 2.4 (II) or (III), we have $f_{1} \simeq \delta_{\mathcal{B}_{1}} \simeq 0$ and $f_{2} \simeq \delta_{\mathcal{B}_{2}} \simeq 0$. Hence it holds that $f=f_{1}+f_{2} \simeq 0$. Thus we obtain (2.14) with $\mathcal{L}=\emptyset$.

(ii). Suppose that $f_{1}$ is represented as $f_{1}(x)=\sum_{i<j} a_{i j} x_{i} x_{j}$ on $U_{n, r}$. Note that $a_{i j}$ is not necessarily finite. We can assume that $\left(a_{i j}\right)$ satisfies the anti-ultrametric property and $\min _{i, j} a_{i j}=0$. Indeed, by Lemma 2.6, $\left(a_{i j}-b_{i}-b_{j}-\alpha^{*}\right)_{i, j \in[n]}$ satisfies the anti-ultrametric property and $\min _{i, j}\left(a_{i j}-b_{i}-b_{j}-\alpha^{*}\right)=$ 
0 for some $b_{i}(i \in[n])$ and $\alpha^{*} \in \mathrm{R}$. Hence

$$
\begin{aligned}
f_{1}(x) & =\sum_{i<j}\left(a_{i j}-b_{i}-b_{j}-\alpha^{*}\right) x_{i} x_{j}+\sum_{i}(r-1) b_{i} x_{i}+\frac{r(r-1) \alpha^{*}}{2} \\
& \simeq \sum_{i<j}\left(a_{i j}-b_{i}-b_{j}-\alpha^{*}\right) x_{i} x_{j}
\end{aligned}
$$

on $\operatorname{dom} \delta_{\mathcal{A}}$. Thus we can redefine $a_{i j} \leftarrow a_{i j}-b_{i}-b_{j}-\alpha^{*}$ for distinct $i, j \in[n]$ to satisfy the anti-ultrametric property and $\min _{i, j} a_{i j}=0$.

Since $\left(a_{i j}\right)$ satisfies the anti-ultrametric property, by Lemma 2.5 , there are a $\mathcal{B}_{1}$-laminar family $\mathcal{L}_{1}$ and a positive weight $c_{1}$ on $\mathcal{L}_{1}$ representing $\left(a_{i j}\right)$ as (2.13). Hence it holds that

$$
\begin{aligned}
f_{1}(x) & =\sum_{L \in \mathcal{L}_{1}} c_{1}(L) \sum_{i, j \in L, i<j} x_{i} x_{j}+\delta_{\mathcal{B}_{1}}(x) \\
& =\sum_{L \in \mathcal{L}_{1}} c_{1}(L) \ell_{L}(x)+\delta_{\mathcal{B}_{1}}(x) \\
& \simeq \sum_{L \in \mathcal{L}_{1}, L: \mathcal{A} \text {-cut }} c_{1}(L) \ell_{L}(x)+\delta_{\mathcal{B}_{1}}(x),
\end{aligned}
$$

where the equivalence follows from Lemma 2.1 (1). Let $\hat{A}_{1}:=\bigcup_{A \in \mathcal{B}_{1}} A$ be the subset of $[n]$ corresponding to $\mathcal{B}_{1}$. By the $\mathcal{B}_{1}$-laminarity of $\mathcal{L}_{1}$, every $L \in \mathcal{L}_{1}$ satisfies $L \supseteq B$ or $L \cap B=\emptyset$ for each $B \in \mathcal{B}_{1} \subseteq \mathcal{A}$. Hence, by Lemma $2.2(1)$,

$$
\ell_{L} \simeq \ell_{L \backslash \hat{A}_{1}} \quad(L \in \mathcal{L})
$$

By combining (2.15) and (2.16), we obtain

$$
f_{1} \simeq \sum_{L \in \mathcal{L}_{1}^{*}} c_{1}^{*}(L) \ell_{L},
$$

where $\mathcal{L}_{1}^{*}:=\left\{L \backslash \hat{A}_{1} \mid L \in \mathcal{L}_{1}, L: \mathcal{A}\right.$-cut $\}$ and $c_{1}^{*}(L):=\sum\left\{c_{1}\left(L^{*}\right) \mid L^{*} \backslash \hat{A}_{1}=L\right\}$. Note that $\mathcal{L}_{1}^{*}$ is a laminar $\mathcal{A}$-cut family and $c_{1}^{*}$ is an aggregation of $c_{1}$.

On the other hand, by Lemma 2.4 (II) or (III), it holds $f_{2} \simeq 0$. Hence, by (2.17), it holds that

$$
f=f_{1}+f_{2} \simeq \sum_{L \in \mathcal{L}_{1}^{*}} c_{1}^{*}(L) \ell_{L} .
$$

Thus, by the laminarity of $\mathcal{A}$-cut family $\mathcal{L}_{1}^{*}$, we obtain (2.14) with $\mathcal{L}=\mathcal{L}^{*}$ and $c=c_{1}^{*}$.

(iii). By the same argument as in (ii), $f_{1}$ satisfies (2.17) and $f_{2}$ satisfies

$$
f_{2} \simeq \sum_{L \in \mathcal{L}_{2}^{*}} c_{2}^{*}(L) \ell_{L},
$$

where $\hat{A}_{2}:=\bigcup_{A \in \mathcal{B}_{2}} A, \mathcal{L}_{2}^{*}:=\left\{L \backslash \hat{A}_{2} \mid L \in \mathcal{L}_{2}, L: \mathcal{A}\right.$-cut $\}$, and $c_{2}^{*}(L):=\sum\left\{c_{2}\left(L^{*}\right) \mid L^{*} \backslash \hat{A}_{2}=L\right\}$ for a $\mathcal{B}_{2}$-laminar family $\mathcal{L}_{2}$ and a positive weight $c_{2}$ on $\mathcal{L}_{2}$. Note that $\mathcal{L}_{2}^{*}$ is a laminar $\mathcal{A}$-cut family. We have $\hat{A}_{2}=[n] \backslash \hat{A}_{1}$ by $\mathcal{B}_{1} \cap \mathcal{B}_{2}=\emptyset$ and $\mathcal{B}_{1} \cup \mathcal{B}_{2}=\mathcal{A}$.

By adding (2.17) and (2.18), it holds

$$
f_{1}+f_{2} \simeq \sum_{L \in \mathcal{L}_{1}^{*}} w_{1}^{*}(L) \ell_{L}+\sum_{L \in \mathcal{L}_{2}^{*}} w_{2}^{*}(L) \ell_{L} .
$$

Hence we obtain (2.14) with $\mathcal{L}=\mathcal{L}_{1}^{*} \cup \mathcal{L}_{2}^{*}$ and $c=c_{1}+c_{2}$, where $\left(c_{1}+c_{2}\right)(L)=c_{1}(L)$ for $L \in \mathcal{L}_{1}^{*}$ and $\left(c_{1}+c_{2}\right)(L)=c_{2}(L)$ for $L \in \mathcal{L}_{2}^{*}$. Here $\mathcal{L}_{1}^{*} \cup \mathcal{L}_{2}^{*}$ is a laminar $\mathcal{A}$-cut family. Indeed, $\mathcal{L}_{1}^{*}$ and $\mathcal{L}_{2}^{*}$ 
are laminar $\mathcal{A}$-cut families, and $L_{1} \cap L_{2}=\emptyset$ holds for all $L_{1} \in \mathcal{L}_{1}^{*}$ and $L_{2} \in \mathcal{L}_{2}^{*}$ by $L_{1} \subseteq[n] \backslash \hat{A}_{1}$ and $L_{2} \subseteq[n] \backslash \hat{A}_{2}=\hat{A}_{1}$.

This completes the proof of Proposition 2.7.

\subsection{Proof of Theorem 2.3: Uniqueness}

In this subsection, we prove the uniqueness of $\mathcal{F}$ and $c$ up to the $\mathcal{A}$-equivalence in Theorem 2.3. Let $f$ be a VCSP-quadratic function of type $\mathcal{A}$. We denote by $\overline{U_{\mathcal{A}}}$ the convex hull of $U_{\mathcal{A}}$, i.e., $\overline{U_{\mathcal{A}}}=\left\{x \in[0,1]^{n} \mid \sum_{i \in A_{p}} x_{i}=1\right.$ for all $\left.p \in[r]\right\}$. The convex closure $\bar{f}: \overline{U_{\mathcal{A}}} \rightarrow \mathbf{R}$ of $f$ is the maximum convex function satisfying $\bar{f}(x)=f(x)$ for $x \in U_{\mathcal{A}}$, which is given by

$$
\bar{f}(x):=\sup \left\{\sum_{1 \leq i \leq n} u_{i} x_{i}+\gamma \mid u \in \mathbf{R}^{n}, \gamma \in \mathbf{R}, f(y) \geq \sum_{1 \leq i \leq n} u_{i} y_{i}+\gamma \quad\left(y \in U_{\mathcal{A}}\right)\right\} .
$$

We first give another representation of $\ell_{X}$ up to the $\mathcal{A}$-linear equivalence. For an $\mathcal{A}$-cut $X$, define

$$
\begin{aligned}
& \alpha(X):=\text { the number of elements } A_{p} \in \mathcal{A} \text { with } X \supseteq A_{p}, \\
& \beta(X):=\text { the number of elements } A_{p} \in \mathcal{A} \text { with } X \cap A_{p} \neq \emptyset .
\end{aligned}
$$

Note that, for any $x \in U_{\mathcal{A}}$ with $\sum_{i \in X} x_{i}=s$, it holds $\sum_{i \in\langle X\rangle \cap X} x_{i}=s-\alpha(X)$ and $\sum_{i \in\langle X\rangle \backslash X} x_{i}=$ $\beta(X)-s$.

LEMMA 2.8. For an $\mathcal{A}$-cut $X$, it holds

$$
\ell_{X}(x) \simeq \frac{1}{2} \sum_{\alpha(X)<k<\beta(X)}\left|k-\sum_{i \in X} x_{i}\right| .
$$

Proof. For the left-hand side of (2.19), it holds $\ell_{X} \simeq\left(\ell_{\langle X\rangle \cap X}+\ell_{\langle X\rangle \backslash X}\right) / 2$ by Lemma 2.2 (2). For the right-hand side of (2.19), we can see that

$$
\sum_{\alpha(X)<k<\beta(X)}\left|k-\sum_{i \in X} x_{i}\right|=\ell_{\langle X\rangle \cap X}(x)+\ell_{\langle X\rangle \backslash X}(x) \quad\left(x \in U_{\mathcal{A}}\right),
$$

and this implies (2.19). Here (2.20) can be established as follows. For $x \in U_{\mathcal{A}}$ with $\sum_{i \in\langle X\rangle \cap X} x_{i}=s$, we have

$$
\begin{aligned}
\sum_{\alpha(X)<k<\beta(X)}\left|k-\sum_{i \in X} x_{i}\right| & =\sum_{\alpha(X)+1 \leq k \leq s}(s-k)+\sum_{s \leq k \leq \beta(X)-1}(k-s) \\
& =\frac{1}{2}((s-\alpha(X))(s-\alpha(X)-1)+(\beta(X)-s)(\beta(X)-s-1)) .
\end{aligned}
$$

On the other hand, by $\sum_{i \in\langle X\rangle \cap X} x_{i}=s-\alpha(X)$ and $\sum_{i \in\langle X\rangle \backslash X} x_{i}=\beta(X)-s$, we have

$$
\begin{aligned}
\ell_{\langle X\rangle \cap X}(x)+\ell_{\langle X\rangle \backslash X}(x) & =\left(\begin{array}{c}
s-\alpha(X) \\
2
\end{array}\right)+\left(\begin{array}{c}
\beta(X)-s \\
2
\end{array}\right) \\
& =\frac{1}{2}((s-\alpha(X))(s-\alpha(X)-1)+(\beta(X)-s)(\beta(X)-s-1)) .
\end{aligned}
$$

Suppose that $f$ is an $\mathrm{M}_{2}$-convex function. By Proposition 2.7 and Lemma 2.8, $f$ is represented as

$$
f(x)=\sum_{L \in \mathcal{L}} \frac{c(L)}{2} \sum_{\alpha(X)<k<\beta(X)}\left|k-\sum_{i \in X} x_{i}\right|+\sum_{1 \leq i \leq n} u_{i} x_{i}+\gamma \quad\left(x \in U_{\mathcal{A}}\right)
$$


for some laminar $\mathcal{A}$-cut family $\mathcal{L}$, positive weight $c$ on $\mathcal{L}$, linear coefficient $u \in \mathbf{R}^{n}$, and constant $\gamma \in \mathbf{R}$. Then $\bar{f}$ is explicitly written as follows.

LEMMA 2.9.

$$
\bar{f}(x)=\sum_{L \in \mathcal{L}} \frac{c(L)}{2} \sum_{\alpha(X)<k<\beta(X)}\left|k-\sum_{i \in X} x_{i}\right|+\sum_{1 \leq i \leq n} u_{i} x_{i}+\gamma \quad\left(x \in \overline{U_{\mathcal{A}}}\right) .
$$

Proof. We denote by $\hat{f}$ the right-hand side of (2.21). It is clear that $f(x)=\hat{f}(x)$ for $x \in \operatorname{dom} f$. Since $\hat{f}$ is piecewise linear and convex, $\hat{f}(z) \leq \bar{f}(z)$ for $z \in \overline{U_{\mathcal{A}}}$ by the definition of $\bar{f}$. Thus it suffices to show $\hat{f}(z) \geq \bar{f}(z)$ for $z \in \overline{U_{\mathcal{A}}}$.

Take any $z \in \overline{U_{\mathcal{H}}}$. Then $z$ satisfies the following system of inequalities and equations for some integers $k_{L}$ for all $L \in \mathcal{L}$ :

$$
\begin{aligned}
& 0 \leq z_{i} \leq 1 \quad(i \in[n]), \\
& \sum_{i \in A_{p}} z_{i}=1 \quad(p \in[r]), \\
& k_{L}-1 \leq \sum_{i \in L} z_{i} \leq k_{L} \quad(L \in \mathcal{L}) .
\end{aligned}
$$

The coefficient matrix $M$ of the system (2.22)-(2.24) is totally unimodular. Indeed, let $M^{\prime}$ be the $n \times(|\mathcal{L}|+r)$ matrix whose columns are the characteristic vectors of the members of $\mathcal{L}$ and $\left\{A_{1}, A_{2}, \ldots, A_{r}\right\} . M$ is represented as $M=\left(I-I M^{\prime}-M^{\prime}\right)^{\top}$, where $I$ is the $n \times n$ identity matrix Since $\mathcal{L}$ and $\left\{A_{1}, A_{2}, \ldots, A_{r}\right\}$ are laminar, $M^{\prime}$ is totally unimodular [12]; see also [34, Theorem 41.11]. Thus $M$ is also totally unimodular.

Let $P$ be the polyhedron defined by the system (2.22)-(2.24). Then $P$ is an integer polyhedron by the total unimodularity of $M$. Hence all extreme points $y_{i}$ of $P$ belong to $U_{\mathcal{A}}$. By $z \in P$, we have $z=\sum_{i} \lambda_{i} y_{i}$ for some coefficients $\lambda_{i}$ of a convex combination. Therefore $\hat{f}(z)=\sum_{i} \lambda_{i} \hat{f}\left(y_{i}\right)=$ $\sum_{i} \lambda_{i} f\left(y_{i}\right)$ holds, where the first equality follows from the linearity of $\hat{f}$ on $P$. Since $f\left(y_{i}\right)=\bar{f}\left(y_{i}\right)$ and $\bar{f}$ is convex, we obtain $\sum_{i} \lambda_{i} f\left(y_{i}\right)=\sum_{i} \lambda_{i} \bar{f}\left(y_{i}\right) \geq \bar{f}(z)$, and hence $\hat{f}(z) \geq \bar{f}(z)$.

We are ready to show the uniqueness part of Theorem 2.3. Suppose that $f$ is $\mathrm{QR}-\mathrm{M}_{2}$-convex. Recall that, by Proposition 2.7 and Lemma 2.8, $f$ is represented as

$$
f(x)=\sum_{L \in \mathcal{L}} \frac{c(L)}{2} \sum_{\alpha(X)<k<\beta(X)}\left|k-\sum_{i \in X} x_{i}\right|+h
$$

for some laminar $\mathcal{A}$-cut family $\mathcal{L}$, positive weight $c$ on $\mathcal{L}$, and $\mathcal{A}$-linear function $h$. Furthermore we can assume that $\mathcal{L}$ is non-redundant. By Lemma 2.9, the set of nondifferentiable points of $\bar{f}$ (with respect to the set of relative interior points of $\overline{U_{\mathcal{A}}}$ ) is given by

$$
\bigcup_{L \in \mathcal{L}, \alpha(L)<k<\beta(L)}\left\{x \in U_{\mathcal{A}} \mid \sum_{i \in L} x_{i}=k\right\}=: P(\mathcal{L}) .
$$

Suppose, to the contrary, that there is another $\left(\mathcal{L}^{\prime}, c^{\prime}\right)$ with $\mathcal{L} \nsim \mathcal{L}^{\prime}$ or $c \nsim c^{\prime}$ that satisfies the conditions in Theorem 2.3, and assume that $\mathcal{L}^{\prime}$ is non-redundant, i.e., $|\mathcal{L}|=\left|\mathcal{L}^{\prime}\right|$.

If $\mathcal{L} \nsim \mathcal{L}^{\prime}$, then there is $L \in \mathcal{L}$ such that $L \nsim L^{\prime}$ for all $L^{\prime} \in \mathcal{L}^{\prime}$. For a set $X \subseteq[n]$, denote by $1_{X} \in\{0,1\}^{n}$ the characteristic vector of $X$. We can easily see that, for $\mathcal{A}$-cut $X$ with $X \nsim L$, 0-1 vectors $1_{A_{1}}, \ldots, 1_{A_{r}}, 1_{L}, 1_{X}$ are linearly independent. Hence, for $k$ with $\alpha(L)<k<\beta(L)$, the dimension of $\left\{x \in \overline{U_{\mathcal{A}}} \mid \sum_{i \in L} x_{i}=k\right\}$ is larger than that of $\left\{x \in \overline{U_{\mathcal{A}}} \mid \sum_{i \in L} x_{i}=k, \sum_{i \in X} x_{i}=k^{\prime}\right\}$ 
for each $k^{\prime}$ with $\alpha(X)<k^{\prime}<\beta(X)$. This implies $\bigcup_{\alpha(L)<k<\beta(L)}\left\{x \in \overline{U_{\mathcal{A}}} \mid \sum_{i \in L} x_{i}=k\right\} \nsubseteq P\left(\mathcal{L}^{\prime}\right)$, and hence $P(\mathcal{L}) \neq P\left(\mathcal{L}^{\prime}\right)$. Therefore $\bar{f}$ has two different sets of nondifferentiable points $P(\mathcal{L})$ and $P\left(\mathcal{L}^{\prime}\right)$, a contradiction. Hence $\mathcal{L} \sim \mathcal{L}^{\prime}$ holds, and we can assume $\mathcal{L}=\mathcal{L}^{\prime}$. If $c \nsim c^{\prime}$, i.e., $c \neq c^{\prime}$, then there is $L \in \mathcal{L}$ such that $c(L) \neq c^{\prime}(L)$. By assuming $c(L)>c^{\prime}(L)(>0)$, we can easily see that $\bar{f}-c^{\prime}(L) \ell_{L}$ has two different sets $P(\mathcal{L})$ and $P(\mathcal{L} \backslash\{L\})$ of nondifferentiable points, a contradiction. Hence $c(L)=c^{\prime}(L)$ holds for all $L \in \mathcal{L}$.

We have thus proved the uniqueness part of Theorem 2.3.

\section{ALGORITHM FOR DECOMPOSITION}

Let $f$ be a VCSP-quadratic function of type $\mathcal{A}=\left\{A_{1}, A_{2}, \ldots, A_{r}\right\}$. In this section, we devise an $O\left(r n^{2}\right)$-time algorithm for Decomposition, where as before $n=\sum_{1 \leq p \leq r}\left|D_{p}\right|$.

\subsection{Outline}

To describe our algorithm, we need the concept of restriction of a VCSP-quadratic function. Recall that $f$ is represented as (2.1). For $Q \subseteq[r]$, let $\mathcal{A}_{Q}:=\left\{A_{p}\right\}_{p \in Q}$ be the subfamily of $\mathcal{A}$ corresponding to $Q$ and $A_{Q}:=\bigcup_{p \in Q} A_{p}$ be the subset of $[n]$ corresponding to $Q$. The restriction of $f$ to $Q$ is a VCSP-quadratic function $f_{Q}:\{0,1\}^{A_{Q}} \rightarrow \overline{\mathbf{R}}$ of type $\mathcal{A}_{Q}$ defined by

$$
f_{Q}(x):= \begin{cases}\sum_{i, j \in A_{Q}, i<j} a_{i j} x_{i} x_{j}+\sum_{i \in A_{Q}} a_{i} x_{i} & \text { if } \sum_{i \in A_{Q}} x_{i}=|Q|, \\ +\infty & \text { otherwise. }\end{cases}
$$

LEMMA 3.1. If $f$ is $Q R-M_{2}$-convex, so is any of its restriction.

Proof. By Lemma 2.5 and Proposition 2.7, $f$ is representable as $f=f^{\prime}+\delta_{\mathcal{A}}$, where the quadratic coefficient $\left(a_{i j}^{\prime}\right)_{i, j \in[n]}$ of $f^{\prime}$ satisfies the anti-ultrametric property. Then $\left(a_{i j}^{\prime}\right)_{i, j \in A_{Q}}$ also has the antiultrametric property. Therefore (3.1) is naturally viewed as a decomposition of $f_{Q}$ to an M-convex function and an $\mathcal{A}_{Q}$-linear function.

Suppose that $f$ is $\mathrm{QR}-\mathrm{M}_{2}$-convex. Then $f_{Q}$ is also QR- $\mathrm{M}_{2}$-convex by Lemma 3.1. By Theorem 2.3, $f_{Q}$ can be represented as

$$
f_{Q}=\sum_{X \in \mathcal{F}_{Q}} c_{Q}(X) \ell_{X}+h_{Q}
$$

for some laminarizable $\mathcal{A}_{Q}$-cut family $\mathcal{F}_{Q}$, positive weight $c_{Q}$ on $\mathcal{F}_{Q}$, and $\mathcal{A}_{Q}$-linear function $h_{Q}$, where $\ell_{X}$ and $h_{Q}$ are defined on $\{0,1\}^{A_{Q}}$. Furthermore such $\mathcal{F}_{Q}$ and $c_{Q}$ are uniquely determined up to $\sim$.

Our algorithm for Decomposition obtains an appropriate decomposition (3.2) for $Q=\{1,2\},\{1,2,3\}, \ldots,\{1$, in turn as follows.

- In the initial case for $Q=\{1,2\}$, we can obtain the decomposition (3.2) with $\left(\mathcal{F}_{Q}, c_{Q}\right)=$ $\left(\mathcal{L}_{12}, c_{12}\right)$ by executing Algorithm 1 for $f_{12}$ (Section 3.2).

- For each $t \geq 3$, we extend $\left(\mathcal{F}_{[t-1]}, c_{[t-1]}\right)$ to $\left(\mathcal{F}_{[t]}, c_{[t]}\right)$ by Algorithm 2 (Section 3.3), where $\mathcal{F}_{[2]}=\mathcal{L}_{12}$. In order to construct $\left(\mathcal{F}_{[t]}, c_{[t]}\right)$ from $\left(\mathcal{F}_{[t-1]}, c_{[t-1]}\right)$, we use $\left(\mathcal{L}_{i t}, c_{i t}\right)$ for all $i \in[t-1]$, which can be obtained by executing Algorithm 1 for $f_{i t}$.

- We perform the above extension step for $t=3$ to $t=r$. Then we can say that the resulting $\mathcal{A}$-cut family $\mathcal{F}_{[r]}$ is laminarizable, as required. This is described in Algorithm 3 (Section 3.3).

Note that our algorithm may output some decomposition (2.7) even when $f$ is not QR-M $\mathrm{M}_{2}$-convex. In this case, the $\mathcal{A}$-cut family $\mathcal{F}$ output by the algorithm is not laminarizable. 


\subsection{Case of $r=2$}

We consider the Decomposition algorithm for the case of $r=2$, where $\mathcal{A}$ is a bipartition of $[n]$ represented as $\left\{A_{1}, A_{2}\right\}$. Note that $X$ is an $\mathcal{A}$-cut if and only if $X$ satisfies $\emptyset \neq X \cap A_{1} \neq A_{1}$ and $\emptyset \neq X \cap A_{2} \neq A_{2}$, and that two $\mathcal{A}$-cuts $X$ and $Y$ are $\mathcal{A}$-equivalent (i.e., $X \sim Y$ ) if and only if $X=Y$ or $X=[n] \backslash Y$ by Lemma 2.2 (2). Let $f$ be a VCSP-quadratic function of type $\left\{A_{1}, A_{2}\right\}$, and $\left(a_{i j}\right)_{i, j \in[n]}$ be the quadratic coefficient of $f$, where $a_{i j}=a_{j i}$ is always assumed.

Our algorithm makes use of the simple fact that, for any $i^{*} \in[n]$ and $b \in \mathbf{R}$, the modification of the coefficients as $a_{i^{*} j}^{\prime} \leftarrow a_{i^{*} j}-b$ (as well as $a_{j i^{*}}^{\prime} \leftarrow a_{j i^{*}}-b$ ) for all $j \in[n] \backslash\left\{i^{*}\right\}$ does not affect the QR-M $\mathrm{M}_{2}$-convexity of $f$. Indeed, the difference between $\sum_{i<j} a_{i j} x_{i} x_{j}$ and $\sum_{i<j} a_{i j}^{\prime} x_{i} x_{j}$ is an $\mathcal{A}$-linear function since, for $x \in U_{\mathcal{A}}$, it holds

$$
\sum_{i<j} a_{i j} x_{i} x_{j}=\left(\sum_{j: j<i^{*}}\left(a_{j i^{*}}-b\right) x_{j} x_{i^{*}}+\sum_{j: j>i^{*}}\left(a_{i^{*} j}-b\right) x_{i^{*}} x_{j}\right)+\sum_{i, j \in[n] \backslash\left\{i^{*}\right\}, i<j} a_{i j} x_{i} x_{j}+b x_{i^{*}} .
$$

We repeat the above modification of coefficients for $i^{*}=1,2, \ldots, n$ with appropriate choices of $b=b_{1}, b_{2}, \ldots, b_{n} \in \mathbf{R}$. Then we test for the QR-M $\mathrm{M}_{2}$-convexity with reference to the condition (CB) below on a quadratic coefficient $\left(a_{i j}\right)_{i, j \in[n] \text { : }}$

(CB) Let the distinct values of $a_{i j}\left(i \in A_{1}, j \in A_{2}\right)$ be $\alpha_{1}>\alpha_{2}>\cdots>\alpha_{m}=\min _{i<j} a_{i j}$. For all $\alpha \in\left\{\alpha_{1}, \alpha_{2}, \ldots, \alpha_{m-1}\right\}$, every non-isolated connected component of $G_{\alpha}:=\left(V, E_{\alpha}\right)$ is a complete bipartite graph, where $E_{\alpha}:=\left\{\{i, j\} \mid i \in A_{1}, j \in A_{2}, \alpha \leq a_{i j}\right\}$.

The following lemma gives a sufficient condition for the QR-M $\mathrm{M}_{2}$-convexity of $f$ in (2.1).

Lемма 3.2. If $\left(a_{i j}-b_{i}-b_{j}\right)_{i, j \in[n]}$ satisfies $(\mathrm{CB})$ for some $b_{1}, b_{2}, \ldots, b_{n} \in \mathbf{R}$, then $f$ in (2.1) is $Q R-M_{2}$-convex.

Proof. Let $f^{\prime}$ be defined by the quadratic coefficient $\left(a_{i j}-b_{i}-b_{j}\right)$ as in (2.1). Then $f$ is $Q R-\mathrm{M}_{2}-$ convex if and only if $f^{\prime}$ is QR-M $\mathrm{M}_{2}$-convex. For each $s \in[m-1]$, denote by $\mathcal{L}^{s}$ the set of non-isolated connected components $L$ of $G_{\alpha_{s}}$. Their union $\mathcal{L}:=\bigcup_{s=1}^{m-1} \mathcal{L}^{s}$ is a laminar family. For $L \in \mathcal{L}$, denote by $L^{+}$the minimal element in $\mathcal{L} \cup\{[n]\}$ properly containing $L$. We define $\alpha_{L}$ for $L \in \mathcal{L} \cup\{[n]\}$ as follows: $\alpha_{[n]}:=\alpha_{m}$ and $\alpha_{L}:=\alpha_{s}$ if $L \in \mathcal{L}^{s} \backslash \mathcal{L}^{s-1}$ with $s \in[m-1]$, where $\mathcal{L}^{0}:=\emptyset$. Since $\left(a_{i j}-b_{i}-b_{j}\right)$ satisfies $(\mathrm{CB})$, we have

$$
\begin{aligned}
\sum_{i<j}\left(a_{i j}-b_{i}-b_{j}\right) x_{i} x_{j} & =\sum_{L \in \mathcal{L}}\left(\alpha_{L}-\alpha_{L^{+}}\right) \ell_{L}(x)+\alpha_{m} \\
& \simeq \sum_{L \in \mathcal{L}^{*}}\left(\alpha_{L}-\alpha_{L^{+}}\right) \ell_{L}(x),
\end{aligned}
$$

where $\mathcal{L}^{*}$ is the family of $\mathcal{A}$-cuts in $\mathcal{L}$. We have thus obtained a representation of $f^{\prime}$ in the form of (2.6) with a laminar $\mathcal{A}$-cut family $\mathcal{L}^{*}$ and a positive weight $c(L)=\alpha_{L}-\alpha_{L^{+}}$on $\mathcal{L}^{*}$. Then $f^{\prime}$ is $\mathrm{QR}-\mathrm{M}_{2}$-convex by Theorem 2.3, and hence $f$ is $\mathrm{QR}-\mathrm{M}_{2}$-convex.

The Decomposition algorithm for the case of $r=2$ is described as Algorithm 1 below. The validity of this algorithm (Proposition 3.4) implies that the converse of Lemma 3.2 is also true, that is, if $f$ is QR-M $\mathrm{M}_{2}$-convex then $\left(a_{i j}-b_{i}-b_{j}\right)_{i, j \in[n]}$ satisfies (CB) by appropriate $b_{i}$ 's, and that such $b_{i}$ 's can be computed easily.

\section{Algorithm 1 (for Decomposition in the case of $r=2$ ):}

Input: A VCSP-quadratic function $f$ of type $\left\{A_{1}, A_{2}\right\}$.

Step 0: Define $\alpha^{*}:=\min _{i, j \in[n], i<j} a_{i j}$.

Step 1: For $i=1,2, \ldots, n$, do the following: Define $b_{i}:=\min _{j \in[n] \backslash\{i\}} a_{i j}-\alpha^{*}$, and update $a_{i j} \leftarrow a_{i j}-b_{i}$ (as well as $a_{j i} \leftarrow a_{j i}-b_{i}$ ) for $j \in[n] \backslash\{i\}$. Then go to next $i$. 

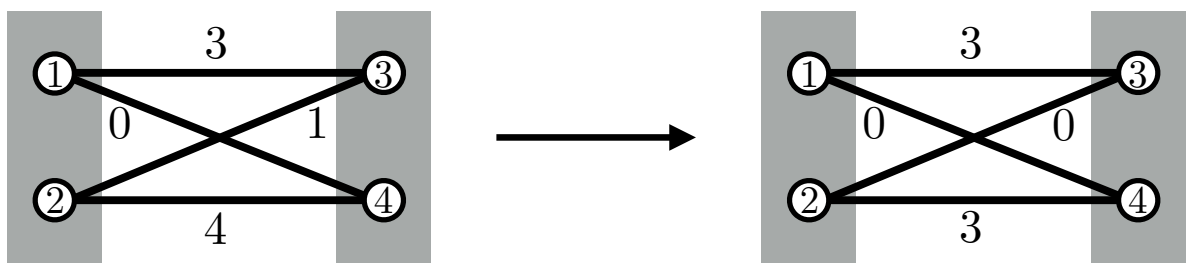

Fig. 2. The left figure illustrates the values of $a_{13}, a_{14}, a_{23}, a_{24}$ before Step 1 , and the right figure illustrates those values after Step 1.

Step 2: Check that $\left(a_{i j}\right)_{i, j \in[n]}$ satisfies (CB) or not. If $\left(a_{i j}\right)_{i, j \in[n]}$ does not satisfy (CB), then output " $f$ is not QR-M $M_{2}$-convex" and stop. If $\left(a_{i j}\right)_{i, j \in[n]}$ satisfies (CB), define $\alpha_{1}>\alpha_{2}>\cdots>\alpha_{m}$ and $G_{\alpha}$ as in the condition (CB).

Step 3: For each $s \in[m-1]$, denote by $\mathcal{L}^{s}$ the set of non-isolated connected components $L$ of $G_{\alpha_{s}}$. Define a laminar family $\mathcal{L}$ by $\mathcal{L}:=\bigcup_{s=1}^{m-1} \mathcal{L}^{s}$. For $L \in \mathcal{L}$, denote by $L^{+}$the minimal element in $\mathcal{L} \cup\{[n]\}$ properly containing $L$. Define $\alpha_{L}$ for $L \in \mathcal{L} \cup\{[n]\}$ by: $\alpha_{[n]}:=\alpha_{m}$ and $\alpha_{L}:=\alpha_{s}$ if $L \in \mathcal{L}^{s} \backslash \mathcal{L}^{s-1}$ with $s \in[m-1]$, where $\mathcal{L}^{0}:=\emptyset$. Define $c: \mathcal{L} \rightarrow \mathbf{R}_{++}$by $c(L):=\alpha_{L}-\alpha_{L^{+}}$.

Step 4: If both $X$ and $[n] \backslash X$ belong to $\mathcal{L}$, then update $c$ by $c(X) \leftarrow c(X)+c([n] \backslash X)$ and remove $[n] \backslash X$ from $\mathcal{L}$. We consider that the new $c$ is a weight on the new $\mathcal{L}$.

Step 5: Output $\mathcal{L}$ and $c$.

Note that, by Step 4, the output $\mathcal{L}$ is non-redundant.

Example 3.3. For the function $f$ in (1.4), we execute Algorithm 1 for the restriction $f_{12}$ to $\{1,2\}$. Recall that $n=4, a_{1}=a_{4}=0, a_{2}=a_{3}=1, a_{13}=3, a_{14}=0, a_{23}=1, a_{24}=4$, and $a_{12}=a_{34}=+\infty$.

In Step 0, we define $\alpha^{*}:=0$. In Step 1, we update $a_{23} \leftarrow 0$ and $a_{24} \leftarrow 3$ (see Figure 2). We can easily see, by Figure 2, $\mathcal{L}=\{13,24\}, \alpha_{13}=\alpha_{24}=3$, and $\alpha_{1234}=0$ in Step 3. In Step 4, we redefine $\mathcal{L}$ by $\mathcal{L}:=\{24\}$ and $c: \mathcal{L} \rightarrow \mathbf{R}_{++}$by $c(24):=6$. Then, in Step 5 , we output $\mathcal{L}$ and $c$. Note that, in Step 4 , we can also redefine $\mathcal{L}$ by $\mathcal{L}:=\{13\}$ and $c: \mathcal{L} \rightarrow \mathbf{R}_{++}$by $c(13):=6$.

Proposition 3.4. Algorithm 1 solves Decomposition in $O\left(n^{2}\right)$ time.

For the proof of the validity of Algorithm 1, we need the following lemma.

Lемма 3.5 ([19, Lемма 4.2]). Suppose that $\left(a_{i j}\right)_{i, j \in[n]}$ satisfies the anti-tree metric property (2.9) and let $\alpha^{*}:=\min _{i, j \in[n], i<j} a_{i j}$. If $\min _{j \in[n]} a_{i j}=\alpha^{*}$ holds for all $i \in[n]$, then $\left(a_{i j}\right)_{i, j \in[n]}$ satisfies the anti-ultrametric property (2.12).

Proof of Proposition 3.4. (Validity). We show that

- if $f$ is not QR-M $\mathrm{M}_{2}$-convex, the algorithm terminates in Step 2, with the message that $f$ is not $\mathrm{QR}-\mathrm{M}_{2}$-convex, and

- if $f$ is QR-M $\mathrm{M}_{2}$-convex, the algorithm terminates in Step 5, with a correct representation of $f$ in the form (2.6).

This means, in particular, that the algorithm for $r=2$ always detects non-QR-M $\mathrm{M}_{2}$-convexity, and never outputs a representation (2.7) with a non-laminarizable family $\mathcal{F}$ if $f$ is not $Q R-\mathrm{M}_{2}$-convex.

Suppose that $f$ is not QR-M $\mathrm{M}_{2}$-convex. By (the contrapositive of) Lemma 3.2, $\left(a_{i j}\right)$ in Step 2 does not satisfy $(\mathrm{CB})$. Accordingly, the algorithm terminates in Step 2, which is legitimate.

Suppose that $f$ is $\mathrm{QR}-\mathrm{M}_{2}$-convex. In this case, $\left(a_{i j}\right)$ in Step 2 satisfies $(\mathrm{CB})$, which is shown in Claim below. Then the algorithm terminates in Step 5 by outputting $(\mathcal{L}, c)$. The laminar family $\mathcal{L}$, 
obtained in Step 3, is an $\mathcal{A}$-cut family. Indeed, by the operation in Step 1,

$$
\min _{j^{\prime} \in A_{2}} a_{i j^{\prime}}=\min _{i^{\prime} \in A_{1}} a_{i^{\prime} j}=\alpha^{*}
$$

holds for any $i \in A_{1}$ and $j \in A_{2}$. This implies that each $L \in \mathcal{L}$ is an $\mathcal{A}$-cut, since otherwise $\min _{j^{\prime} \in[n] \backslash\{i\}} a_{i j^{\prime}}>\alpha^{*}$ holds for some $i \in L$. Therefore, by the proof of Lemma 3.2, the output $(\mathcal{L}, c)$ gives a correct representation of $f$ in the form (2.6).

It remains to prove the following claim.

CLAIm. If $f$ is $Q R-M_{2}$-convex, then $\left(a_{i j}\right)_{i, j \in[n]}$ in Step 2 satisfies (CB).

Proof of Claim. Suppose that $f$ is $Q R-M_{2}$-convex. In the following, we prove that there is a coefficient $\left(\bar{a}_{i j}\right)$ satisfying the anti-ultrametric property such that $a_{i j}=\bar{a}_{i j}$ for every $i \in A_{1}$ and $j \in A_{2}$, where it should be clear that $a_{i j}=+\infty$ if $i, j \in A_{1}$ or $i, j \in A_{2}$. This implies, by Lemma 2.5, that there are a laminar family $\mathcal{L}$ and a positive weight $w$ on $\mathcal{L}$ associated with $\left(\bar{a}_{i j}\right)$ as (2.13). Then $a_{i j}$ can be represented as

$$
a_{i j}= \begin{cases}\{c(L) \mid L \in \mathcal{L} \text { with } i, j \in L\}+\alpha^{*} & \text { if } i \in A_{1} \text { and } j \in A_{2}, \\ +\infty & \text { if } i, j \in A_{1} \text { or } i, j \in A_{2} .\end{cases}
$$

Hence $\left(a_{i j}\right)$ satisfies $(\mathrm{CB})$ and the laminar family obtained in Step 3 coincides with the family of $\mathcal{A}$-cuts in $\mathcal{L}$.

We now start to prove the existence of $\left(\bar{a}_{i j}\right)$. By Lemma 2.6 and Proposition 2.7, we have $f(x) \simeq$ $\sum_{i<j} a_{i j}^{\prime} x_{i} x_{j}$, where $\left(a_{i j}^{\prime}\right)$ is a coefficient satisfying the anti-ultrametric property. This implies that $\sum_{i<j} a_{i j} x_{i} x_{j}-\sum_{i<j} a_{i j}^{\prime} x_{i} x_{j}$ is $\mathcal{A}$-linear. Hence, for some $b_{i}^{\prime}, b_{j}^{\prime} \in \mathrm{R}$ we have $a_{i j}=a_{i j}^{\prime}+b_{i}^{\prime}+b_{j}^{\prime}$ for every $i \in A_{1}, j \in A_{2}$. Let $\bar{a}_{i j}:=a_{i j}^{\prime}+b_{i}^{\prime}+b_{j}^{\prime}$ for distinct $i, j \in[n]$. Then $a_{i j}=\bar{a}_{i j}$ holds for any $i \in A_{1}, j \in A_{2}$, and $\left(\bar{a}_{i j}\right)$ is a coefficient satisfying the anti-tree metric property (2.9).

We can redefine the coefficient $\left(\bar{a}_{i j}\right)$ so as to meet the anti-ultrametric property while maintaining $a_{i j}=\bar{a}_{i j}$ for any $i \in A_{1}, j \in A_{2}$, as follows. Let $\beta:=\alpha^{*}-\min \bar{a}_{i j}$. Note that $\beta \geq 0$ holds by (3.3) and $a_{i j}=\bar{a}_{i j}$ for $i \in A_{1}, j \in A_{2}$. First suppose $\beta=0$. Then $\min \bar{a}_{i j}=\alpha^{*}$ holds. Furthermore, we have $\min _{j} \bar{a}_{i j}=\alpha^{*}$ for every $i \in[n]$. Hence, by Lemma 3.5, $\left(\bar{a}_{i j}\right)$ satisfies the anti-ultrametric property, as required.

Next suppose $\beta>0$. By $\bar{a}_{i j} \geq \alpha^{*}$ for any $i \in A_{1}, j \in A_{2}$, if $\bar{a}_{i^{*} j^{*}}=\alpha^{*}-\beta$, then $i^{*}, j^{*} \in A_{1}$ or $i^{*}, j^{*} \in A_{2}$ holds. Without loss of generality, we assume $i^{*}, j^{*} \in A_{1}$. Since $\left(\bar{a}_{i j}\right)$ satisfies (2.9), it holds that $\bar{a}_{i^{*} j^{*}}+\bar{a}_{k l} \geq 2 \alpha^{*}$ for all distinct $k, l \in A_{2}$. Hence we have $\min _{k, l \in A_{2}} \bar{a}_{k l} \geq \alpha^{*}+\beta$. Let $\bar{b}_{i}:=\beta / 2$ if $i \in A_{1}$ and $\bar{b}_{i}:=-\beta / 2$ if $i \in A_{2}$. We redefine $\bar{a}_{i j}$ as $\bar{a}_{i j} \leftarrow \bar{a}_{i j}+\bar{b}_{i}+\bar{b}_{j}$. Then it is easy to see that $a_{i j}=\bar{a}_{i j}$ holds for any $i \in A_{1}, j \in A_{2}$, and that $\left(\bar{a}_{i j}\right)$ is a coefficient satisfying (2.9). Furthermore $\alpha^{*}-\min \bar{a}_{i j}=0$ holds. Hence, by Lemma 3.5, $\left(\bar{a}_{i j}\right)$ satisfies the anti-ultrametric property, as required.

This completes the proof of Claim.

(Complexity). It is clear that Steps 0 and 1 can be done in $O\left(n^{2}\right)$ time, and that Steps 4 and 5 can be done in $O(|\mathcal{L}|)=O(n)$ time.

We show that Steps 2 and 3 can be done in $O\left(n^{2}\right)$ time, improving the $O\left(n^{3}\right)$ time complexity of a naive implementation. Our approach is based on the idea used in [19, Section 4.2.2] (see also [4, 37]). Suppose that $f$ is QR-M $\mathrm{M}_{2}$-convex, and that we are given some $L \in \mathcal{L}$. We can compute in $O\left(|L|^{2}\right)$ time the (disjoint) set $\mathcal{L}^{\prime}$ of all maximal members in $\mathcal{L}$ properly contained in $L$ as follows. Let $L_{1}:=A_{1} \cap L$ and $L_{2}:=A_{2} \cap L$. Observe that $\alpha_{L}=\min _{j^{\prime} \in L_{2}} a_{i j^{\prime}}=\min _{i^{\prime} \in L_{1}} a_{i^{\prime} j}$ holds for each $i \in L_{1}$ and $j \in L_{2}$. Choose arbitrary $i \in L_{1}$. and compute $\operatorname{argmin}_{j^{\prime} \in L_{2}} a_{i j^{\prime}}$. If $a_{i j^{\prime}}$ is constant on $j^{\prime} \in L_{2}$, then there is no member of $\mathcal{L}^{\prime}$ containing $i$. Otherwise, choose $j$ from $L_{2} \backslash \operatorname{argmin}_{j^{\prime} \in L_{2}} a_{i j^{\prime}}$, and compute $\operatorname{argmin}_{i^{\prime} \in L_{1}} a_{i^{\prime} j}$. Then one can see that the (unique) member $L^{\prime}$ in $\mathcal{L}$ containing $i, j$ is equal to the 
union of $L_{1} \backslash \operatorname{argmin}_{i^{\prime} \in L_{1}} a_{i^{\prime} j}$ and $L_{2} \backslash \operatorname{argmin}_{j^{\prime} \in L_{2}} a_{i j^{\prime}}$. By repeating this procedure, we obtain $\mathcal{L}^{\prime}$ in $O\left(|L|^{2}\right)$ time. Thus, starting from $L=[n]$, we recursively apply this procedure to the $L$ 's so far obtained, and finally get $\mathcal{L}$ (as well as $c: \mathcal{L} \rightarrow \mathbf{R}_{++}$) in $O\left(n^{2}\right)$-time in total. Even when $f$ is not $\mathrm{QR}-\mathrm{M}_{2}$-convex, we can apply this procedure and detect the non- $\mathrm{QR}-\mathrm{M}_{2}$-convexity. Indeed, define $a_{i j}^{\prime}$ as $\alpha_{L}$ for the final $L$ containing $i, j$ in the above procedure. Then $a_{i j}^{\prime}=a_{i j}$ holds for any $i, j$ if and only if $\left(a_{i j}\right)$ satisfies the anti-ultrametric property, i.e., $f$ is $\mathrm{QR}-\mathrm{M}_{2}$-convex.

\subsection{Case of $r \geq 3$}

To obtain the decomposition (3.2) of the restriction $f_{Q}$ for $Q=\{1,2\},\{1,2,3\}, \ldots,\{1,2,3, \ldots, r\}$ in turn, we need to extend $\left(\mathcal{F}_{[t-1]}, c_{[t-1]}\right)$ to $\left(\mathcal{F}_{[t]}, c_{[t]}\right)$ with the use of $\left(\mathcal{L}_{p t}, c_{p t}\right)(p \in[t-1])$ for $t=3, \ldots, r$. Algorithm 2 below corresponds to this extension procedure.

We explain the idea of the extension for $t=r$, i.e., from $\left(\mathcal{F}_{[r-1]}, c_{[r-1]}\right)$ to $\left(\mathcal{F}_{[r]}, c_{[r]}\right)$. Suppose that we are given an $\mathcal{A}_{[r-1]}$-cut family $\mathcal{F}^{\prime}$ and a positive weight $c^{\prime}$ on $\mathcal{F}^{\prime}$ satisfying, for $f^{\prime}:=f_{[r-1]}$,

$$
f^{\prime}=\sum_{X \in \mathcal{F}^{\prime}} c^{\prime}(X) \ell_{X}+h^{\prime}
$$

for some $\mathcal{A}_{[r-1]}$-linear function $h^{\prime}$.

The extension procedure consists of two phases. In the first phase, we construct an $\mathcal{A}$-cut family $\mathcal{F}$ and a positive weight $c$ on $\mathcal{F}$ that represent $f$ as

$$
f=\sum_{X \in \mathcal{F}} c(X) \ell_{X}+h
$$

for some $\mathcal{A}$-linear function $h$. In this representation, however, the family $\mathcal{F}$ is not necessarily laminarizable even when $f$ is $Q R-M_{2}$-convex. In the second phase we modify $(\mathcal{F}, c)$ in (3.5) to another pair $\left(\mathcal{F}^{*}, c^{*}\right)$ such that $\mathcal{F}^{*}$ is laminarizable when $f$ is $\mathrm{QR}-\mathrm{M}_{2}$-convex. The key operation of the second phase is called a "composition" operation.

The first phase is easy and straightforward. Suppose that we have a decomposition (3.4) for $f^{\prime}$ in terms of $\left(\mathcal{F}^{\prime}, c^{\prime}\right)$. For $p=1,2, \ldots, r-1$, we apply Algorithm 1 to $f_{p r}$ to obtain the decomposition (3.2) of $f_{p r}$ in terms of $\left(\mathcal{L}_{p r}, c_{p r}\right)$. If Algorithm 1 should detect the non-QR-M $\mathrm{M}_{2}$-convexity of $f_{p r}$ for some $p \in[r-1]$, then $f$ is not QR-M $\mathrm{M}_{2}$-convex by Lemma 3.1, and therefore, we can give up our construction immediately. Otherwise, we merge $\left(\mathcal{F}^{\prime}, c^{\prime}\right)$ and $\left(\mathcal{L}_{p r}, c_{p r}\right)(p \in[r-1])$ to obtain a representation of $f$. Let $\mathcal{F}:=\mathcal{F}^{\prime} \cup \cup_{p \in[r-1]} \mathcal{L}_{p r}$, which is an $\mathcal{A}$-cut family, and define a positive weight $c$ on $\mathcal{F}$ by $c(X):=c^{\prime}(X)$ for $X \in \mathcal{F}^{\prime}$ and $c(X):=c_{p r}(X)$ for $X \in \mathcal{L}_{p r}$. Then, with the notation $\left.x\right|_{Q}:=\left(x_{i}\right)_{i \in A_{Q}} \in\{0,1\}^{A_{Q}}$ for $x=\left(x_{1}, x_{2}, \ldots, x_{n}\right) \in\{0,1\}^{n}$ and $Q \subseteq[r]$, we have

$$
\begin{aligned}
f(x) & \simeq \begin{cases}\sum_{i, j \in A_{[r-1]}, i<j} a_{i j} x_{i} x_{j}+\sum_{p \in[r-1]} \sum_{i, j \in A_{p r}, i<j} a_{i j} x_{i} x_{j} & \text { if } \sum_{i} x_{i}=r \\
+\infty & \text { otherwise }\end{cases} \\
& \simeq f^{\prime}\left(\left.x\right|_{[r-1]}\right)+\sum_{p \in[r-1]} f_{p r}\left(\left.x\right|_{p r}\right) \\
& \simeq \sum_{X \in \mathcal{F}} c(X) \ell_{X} .
\end{aligned}
$$

Thus the representation (3.5) for $f$ is obtained. Recall that we do not impose laminarizability on $\mathcal{F}$ even when $f$ is $\mathrm{QR}-\mathrm{M}_{2}$-convex. As the above argument shows, no substantial computation is required in the first phase.

The second phase consists of modifying $(\mathcal{F}, c)$ in $(3.5)$ to another pair $\left(\mathcal{F}^{*}, c^{*}\right)$ with the additional property that $\mathcal{F}^{*}$ is laminarizable when $f$ is $\mathrm{QR}-\mathrm{M}_{2}$-convex. For this modification we introduce a 

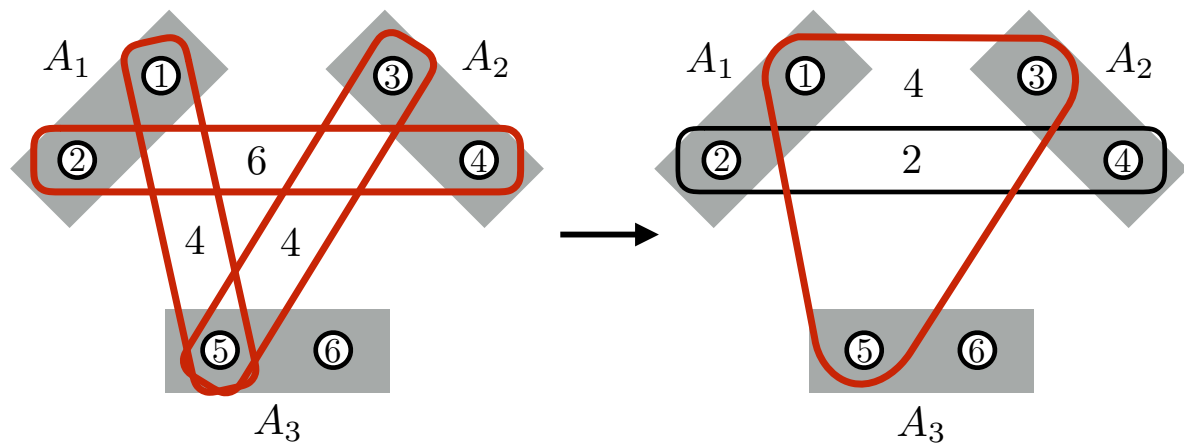

Fig. 3. The left figure illustrates $\left(\mathcal{L}_{12}, c_{12}\right),\left(\mathcal{L}_{13}, c_{13}\right)$, and $\left(\mathcal{L}_{23}, c_{23}\right)$, and the right figure illustrates $\left(\mathcal{F}_{123}, c_{123}\right)$. A pair $(15,35)$ is a composable tuple to 24 since 135 satisfies $135 \sim_{12} 24,135 \sim_{13} 15$, and $135 \sim_{23} 35$
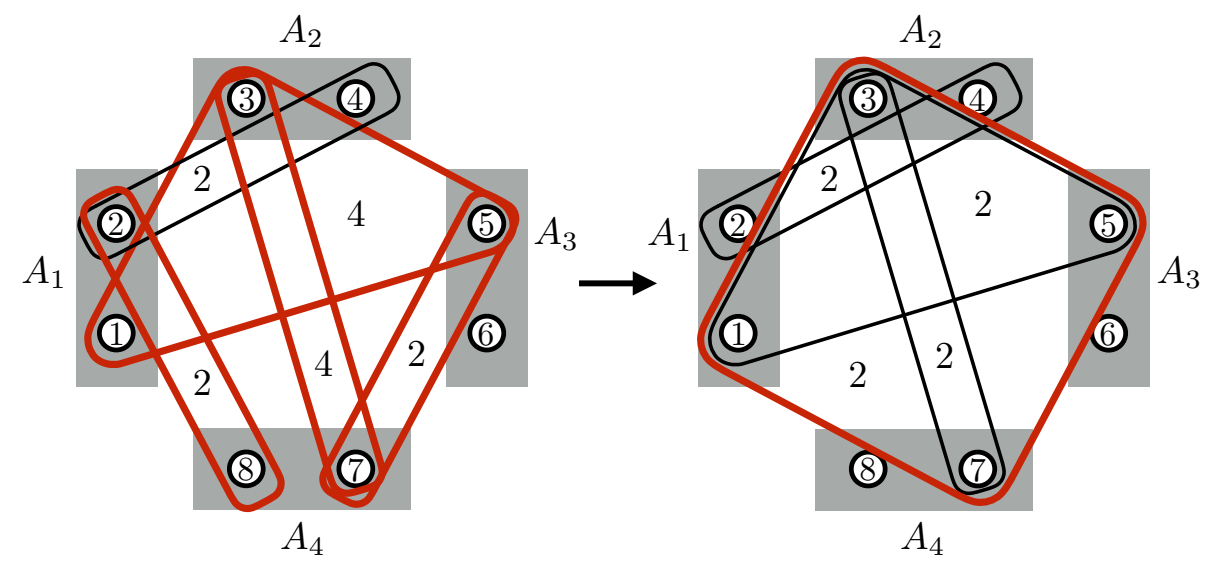

Fig. 4. The left figure illustrates $\left(\mathcal{F}_{123}, c_{123}\right),\left(\mathcal{L}_{14}, c_{14}\right),\left(\mathcal{L}_{24}, c_{24}\right)$, and $\left(\mathcal{L}_{34}, c_{34}\right)$ and the right figure illustrates $(\mathcal{F}, c)$. A triple $(28,37,57)$ a composable tuple to 135 since 1357 satisfies $1357 \sim_{123} 135,1357 \sim_{14} 28,1357 \sim_{24}$ 37 , and $1357 \sim_{34} 57$. Note that the output family $\mathcal{F}$ (described in the right) is the same as the family described in the left in Figure 1.

"composition" operation. Before entering into a formal description, we illustrate this modification for simple examples in Figures 3 and 4 . In Figure 3, the given family $\mathcal{F}=\{24,15,35\}$ at the left is not laminar and the resulting family $\mathcal{F}^{*}=\{135,24\}$ at the right is laminar; the new $\mathcal{A}$ cut $X^{*}=135$ is constructed by our algorithm by combining 24, 15, and 35. In Figure 4, the given family $\mathcal{F}=\{135,24,28,37,57\}$ at the left is not laminarizable and the resulting family $\mathcal{F}^{*}=\{135,24,1357,37\}$ at the right is not laminar but laminarizable; the new $\mathcal{A}$-cut $X^{*}=1357$ is constructed by our algorithm by combining 135, 28, 37, and 57 .

In order to explain the composition operation, we introduce the $\mathcal{A}_{Q}$-equivalence $\sim_{Q}$ by generalizing the characterization of $\sim$ in (2.4). For a nonempty $Q \subseteq[r]$, we define $\sim_{Q}$ for $\mathcal{A}$-cuts $X$ and $Y$ by:

$$
X \sim_{Q} Y \Leftrightarrow\left\{\langle X\rangle_{Q} \cap X,\langle X\rangle_{Q} \backslash X\right\}=\left\{\langle Y\rangle_{Q} \cap Y,\langle Y\rangle_{Q} \backslash Y\right\}
$$

where $\langle X\rangle_{Q}:=\langle X\rangle \cap A_{Q}$ and $\langle Y\rangle_{Q}:=\langle Y\rangle \cap A_{Q}$. See (2.3) for the notation $\langle X\rangle$ of the cutting support of $X$. Note, for $\mathcal{A}_{Q}$-cuts $X$ and $Y, X \sim Y$ if and only if $X \sim_{Q} Y$. 
Let us start the description of the composition operation. Suppose that $X_{0}$ is an $\mathcal{A}_{[r-1]}$-cut and let $\left\{p_{1}, p_{2}, \ldots, p_{k}\right\}$ be the set of indices $p \in[r-1]$ with $\left\langle X_{0}\right\rangle=A_{\left\{p_{1}, p_{2}, \ldots, p_{k}\right\}}$. We say that $\left(X_{1}, X_{2}, \ldots, X_{k}\right)$ is a composable tuple to $X_{0}$ if

- $\left\langle X_{i}\right\rangle$ is an $\mathcal{A}_{p_{i} r}$-cut (i.e., $\left\langle X_{i}\right\rangle=A_{p_{i} r}$ ) for each $i \in[k]$, and

- there is an $\mathcal{A}$-cut $X^{*}$ satisfying $X^{*} \sim_{[r-1]} X_{0}$ and $X^{*} \sim_{p_{i} r} X_{i}$ for $i \in[k]$.

We say that $X^{*}$ in the second condition is a composition of $X_{0}$ and $\left(X_{1}, X_{2}, \ldots, X_{k}\right)$. Note that a composition $X^{*}$ is uniquely determined up to $\sim$. Then it holds

$$
\ell_{X_{0}}+\ell_{X_{1}}+\cdots+\ell_{X_{k}} \simeq \ell_{\left\langle X_{0}\right\rangle \cap X^{*}}+\ell_{\left\langle X_{1}\right\rangle \cap X^{*}}+\cdots+\ell_{\left\langle X_{k}\right\rangle \cap X^{*}} \simeq \ell_{X^{*}},
$$

where the first equivalence follows from Lemma 2.2 (2) and the second follows form the definition of $\ell_{X}$. Let $\lambda$ be a positive value with $\lambda=\min \left\{c\left(X_{0}\right), c\left(X_{1}\right), \ldots, c\left(X_{k}\right)\right\}$. By substituting (3.6) into (3.5), we obtain

$$
f \simeq\left(\lambda \ell_{X^{*}}+\sum_{X \in\left\{X_{0}, \ldots, X_{k}\right\}}(c(X)-\lambda) \ell_{X}+\sum_{X \in \mathcal{F} \backslash\left\{X_{0}, \ldots, X_{k}\right\}} c(X) \ell_{X}\right),
$$

and the above formula provides a new decomposition of $f$. For example, in Figure 4, we combine $X_{0}=135, X_{1}=28, X_{2}=37, X_{3}=57$ into $X^{*}=1357$ with $\lambda=2$.

The formal description of Algorithm 2 is the following. It is noted that, if $\mathcal{F}$ is a non-redundant laminarizable $\mathcal{A}$-cut family, then $|\mathcal{F}|$ is at most $2 n=2\left|A_{[r]}\right|$ (see e.g., [34, Theorem 3.5]).

Algorithm 2 (for extending $f^{\prime}$ to $f$ ):

Input: A VCSP-quadratic function $f$ of type $\mathcal{A}$ and restriction $f^{\prime}:=f_{[r-1]}$ given as (3.4) with $\left(\mathcal{F}^{\prime}, c^{\prime}\right)$, where $\mathcal{F}^{\prime}$ is non-redundant and satisfies $\left|\mathcal{F}^{\prime}\right| \leq 2\left|A_{[r-1]}\right|$.

Output: Either detect the non-QR- $\mathrm{M}_{2}$-convexity of $f$, or obtain an expression of $f$ as

$$
\sum_{X \in \mathcal{F}} c(X) \ell_{X}+\delta_{\mathcal{A}}+h
$$

with a non-redundant $\mathcal{A}$-cut family $\mathcal{F}$ satisfying $|\mathcal{F}| \leq 2 n=2\left|A_{[r]}\right|$ and a positive weight $c$ on $\mathcal{F}$, where $h$ is $\mathcal{A}$-linear.

Step 1: For each $p \in[r-1]$, execute Algorithm 1 for $f_{p r}$. If Algorithm 1 returns " $f_{p r}$ is not QR$\mathrm{M}_{2}$-convex" for some $p \in[r-1]$, then output " $f$ is not QR-M $\mathrm{M}_{2}$-convex" and stop. Otherwise, for all $p \in[r-1]$, obtain $\mathcal{L}_{p r}$ and $c_{p r}$. Let $\mathcal{F}:=\emptyset$.

Step 2: While $\mathcal{F}^{\prime} \neq \emptyset$, do the following: Let $X_{0}$ be an element of $\mathcal{F}^{\prime}$ such that $\left\langle X_{0}\right\rangle$ is maximal. Let $\left\{p_{1}, p_{2}, \ldots, p_{k}\right\}$ be the set of indices $p \in[r-1]$ with $\left\langle X_{0}\right\rangle=A_{\left\{p_{1}, p_{2}, \ldots, p_{k}\right\}}$.

- If there exists a composable tuple $\left(X_{1}, X_{2}, \ldots, X_{k}\right)$ to $X_{0}$ such that $X_{i} \in \mathcal{L}_{p_{i} k}$ for $i=$ $1,2, \ldots k$, then define $\lambda:=\min \left\{c^{\prime}\left(X_{0}\right), c_{p_{1} r}\left(X_{1}\right), \ldots, c_{p_{k} r}\left(X_{k}\right)\right\}$ and update as

$$
\begin{aligned}
\mathcal{F} & \leftarrow \mathcal{F} \cup\left\{X^{*}\right\}, \\
c\left(X^{*}\right) & \leftarrow \lambda, \\
c^{\prime}\left(X_{0}\right) & \leftarrow c^{\prime}\left(X_{0}\right)-\lambda, \\
c_{p_{i} r}\left(X_{i}\right) & \leftarrow c_{p_{i} r}\left(X_{i}\right)-\lambda \quad(i \in[k]),
\end{aligned}
$$

where $X^{*}$ is a composition of $X_{0}$ and $\left(X_{1}, X_{2}, \ldots, X_{k}\right)$. Then remove $X_{0}$ from $\mathcal{F}^{\prime}$ if $c^{\prime}\left(X_{0}\right)=0$, and $X_{i}$ from $\mathcal{L}_{p_{i} r}$ if $c_{p_{i} r}\left(X_{i}\right)=0$.

- Otherwise, update as

$$
\begin{aligned}
\mathcal{F} & \leftarrow \mathcal{F} \cup\left\{X_{0}\right\}, \\
c\left(X_{0}\right) & \leftarrow c^{\prime}\left(X_{0}\right), \\
\mathcal{F}^{\prime} & \leftarrow \mathcal{F}^{\prime} \backslash\left\{X_{0}\right\} .
\end{aligned}
$$


Step 3: Update as

$$
\begin{aligned}
\mathcal{F} & \leftarrow \mathcal{F} \cup \bigcup_{p \in[r-1]} \mathcal{L}_{p r}, \\
c(X) & \leftarrow c_{p r}(X) \quad\left(p \in[r-1], X \in \mathcal{L}_{p r}\right) .
\end{aligned}
$$

If $|\mathcal{F}| \leq 2 n$, then output $\mathcal{F}$ and $c$. Otherwise, output " $f$ is not $Q R-\mathrm{M}_{2}$-convex."

Example 3.6. Let $f$ be the VCSP-quadratic function in (1.4). We first see how Algorithm 2 runs for $f_{123}$ with the input $\left(\mathcal{L}_{12}=\{24\}, c_{12}(24)=6\right)$. By executing Algorithm 1 for $f_{13}$ and $f_{23}$, we obtain $\left(\mathcal{L}_{13}=\{15\}, c_{13}(15)=4\right)$ and $\left(\mathcal{L}_{23}=\{35\}, c_{23}(35)=4\right)$. In Step 2, we compose 15, 35, 24 to 135 as in Figure 3. Then we obtain a family $\mathcal{F}_{123}:=\{135,24\}$ and a positive weight $c_{123}$ on $\mathcal{F}_{123}$ defined by $c_{123}(135):=4$ and $c_{123}(24):=2$. We cannot execute a composition operation any more. Hence Algorithm 2 outputs $\left(\mathcal{F}_{123}, c_{123}\right)$.

Next we see how Algorithm 2 runs for $f=f_{1234}$ with the input $\left(\mathcal{F}_{123}, c_{123}\right)$. By executing Algorithm 1 for $f_{14}, f_{24}$, and $f_{34}$, we obtain $\left(\mathcal{L}_{14}=\{28\}, c_{14}(28)=2\right),\left(\mathcal{L}_{24}=\{37\}, c_{24}(37)=4\right)$, and $\left(\mathcal{L}_{34}=\{57\}, c_{34}(57)=2\right)$. In Step 2, we compose 135, 28, 37, 57 to 1357 as in Figure 3. Then we obtain a family $\mathcal{F}:=\{1357,135,24,37\}$ and a positive weight $c$ on $\mathcal{F}$ defined by $c(X):=2$ for all $X \in \mathcal{F}$. Here we remark that we choose a composable tuple $(28,37,57)$ to $(135)$ though $(28,37)$ is also a composable tuple to 24 . This is because $\langle 135\rangle \supsetneq\langle 24\rangle$; see Step 2. We cannot execute a composition operation any more. Hence Algorithm 2 outputs $(\mathcal{F}, c)$.

The following proposition shows that Algorithm 2 works as expected.

Proposition 3.7. The following hold:

(1) If Algorithm 2 outputs $(\mathcal{F}, c)$, then $\mathcal{F}$ is non-redundant and the function (3.7) for $(\mathcal{F}, c)$ is equal to $f$.

(2) If $f$ is $Q R-M_{2}$-convex and $\mathcal{F}^{\prime}$ is laminarizable, then Algorithm 2 outputs $(\mathcal{F}, c)$ and $\mathcal{F}$ is laminarizable.

(3) Algorithm 2 runs in $O\left(n^{2}\right)$ time provided $\left|A_{r}\right| \leq \min \left\{\left|A_{1}\right|,\left|A_{2}\right|, \ldots,\left|A_{r-1}\right|\right\}$.

For the proof of Proposition 3.7 (2), we need the following lemma.

Lемма 3.8. Suppose that $f$ is $Q R-M_{2}$-convex. For $Q \subseteq[r]$, let $\mathcal{G}:=\left\{X \cap A_{Q} \mid X \in \mathcal{F}, X \cap A_{Q}\right.$ : $\mathcal{A}_{Q}$-cut $\}$ and $d$ be the positive weight on $\mathcal{G}$ defined byd $(Y):=\sum\left\{c(X) \mid X \in \mathcal{F}, X \cap A_{Q}=Y\right\}$. Then $\mathcal{F}_{Q}$ and $c_{Q}$ in (3.2) satisfy $\mathcal{F}_{Q} \sim \mathcal{G}$ and $c_{Q} \sim d$.

Proof. For an $\mathcal{A}$-cut $X$ and $Q \subseteq[r]$, let $\left(\ell_{X}\right)_{Q}$ be the restriction of $\ell_{X}$ to $\{0,1\}^{A_{Q}}$. Note that $\left(\ell_{X}\right)_{Q}+\delta_{\mathcal{A}_{Q}}$ is linear on $\operatorname{dom} \delta_{\mathcal{A}_{Q}}$ if and only if $X \cap A_{Q}$ is not an $\mathcal{A}_{Q}$-cut. Therefore it holds that

$$
\begin{aligned}
f_{Q} & \simeq \sum_{X \in \mathcal{F}} c(X)\left(\ell_{X}\right)_{Q} \\
& \simeq \sum_{Y \in \mathcal{G}} \ell_{Y} \cdot \sum\left\{c(X) \mid X \in \mathcal{F}, X \cap A_{Q}=Y\right\} \\
& =\sum_{Y \in \mathcal{G}} d(Y) \ell_{Y} .
\end{aligned}
$$

Furthermore, since $\mathcal{F}$ is laminarizable, so is $\mathcal{G}$. By the uniqueness of $\mathcal{F}_{Q}$ and $c_{Q}$ up to $\sim$ (Theorem 2.3), we obtain $\mathcal{F}_{Q} \sim \mathcal{G}$ and $c_{Q} \sim d$.

We are now ready to show Proposition 3.7. 
Proof of Proposition 3.7. (1). By the argument before the formal description of Algorithm 2, we can say that if Algorithm 2 outputs $(\mathcal{F}, c)$, then it constructs some decomposition of $f$. Hence the equality holds. The non-redundancy of $\mathcal{F}$ is clear by its construction.

(2). Since $f$ is QR-M $\mathrm{M}_{2}$-convex, so is $f_{p r}$ for $p \in[r-1]$. Hence, by Proposition 3.4, Algorithm 2 does not output " $f$ is not $Q R-M_{2}$-convex" in Step 1. Let $\mathcal{F}^{*}$ be a non-redundant laminarizable $\mathcal{A}$-cut family and $c^{*}$ be a positive weight on $\mathcal{F}^{*}$ that satisfy (2.6) for the given QR-M $\mathrm{M}_{2}$-convex function $f$ We extend $c^{*}$ to a nonnegative weight on $2^{[n]}$ by defining $c^{*}(X):=0$ for $X \notin \mathcal{F}$. We can assume that if $X \in \mathcal{F}$ and $Y \in \mathcal{F}^{*}$ satisfies $X \sim Y$ then it holds $X=Y$. It suffices to prove (i) $c\left(X^{*}\right)=c^{*}\left(X^{*}\right)$ for $X^{*}$ obtained in the first half of Step 2, (ii) $c\left(X_{0}\right)=c^{*}\left(X_{0}\right)$ for $X_{0}$ obtained in the latter half of Step 2, and (iii) $c(X)=c^{*}(X)$ for $X$ obtained in Step 3. Indeed, the properties (i)-(iii) imply $\mathcal{F} \subseteq \mathcal{F}^{*}$. Since $\mathcal{F}^{*}$ is laminarizable, so is $\mathcal{F}$ and $|\mathcal{F}| \leq 2 n$. Hence Algorithm 2 outputs $(\mathcal{F}, c)$ in Step 3. By the uniqueness $\mathcal{F}^{*}$ under $\sim\left(\right.$ Theorem 2.3), we can say $\mathcal{F}=\mathcal{F}^{*}$ and $c=c^{*}$.

(i). Let $\lambda:=\min \left\{c^{\prime}\left(X_{0}\right), c_{p_{1} r}\left(X_{1}\right), c_{p_{2} r}\left(X_{2}\right), \ldots, c_{p_{k} r}\left(X_{k}\right)\right\}$. We prove $c^{*}\left(X^{*}\right)=\lambda$. It is easy to see that $c^{*}\left(X^{*}\right) \leq \lambda$ holds since, by Lemma 3.8, we have $c^{\prime}\left(X_{0}\right) \geq c^{*}\left(X^{*}\right)$ and $c_{p_{i} r}\left(X_{i}\right) \geq c^{*}\left(X^{*}\right)$ for $i \in[k]$.

Suppose, to the contrary, that $c^{*}\left(X^{*}\right)<\lambda$ holds. Then the following holds:

Claim. Every $Y_{0} \in \mathcal{F}^{*}$ with $Y_{0} \sim_{[r-1]} X^{*}$ satisfies $Y_{0} \sim X^{*}$.

On the other hand, by Lemma 3.8 with $c^{*}\left(X^{*}\right)<\lambda \leq c^{\prime}\left(X_{0}\right)$, there must exist $Y_{0} \in \mathcal{F}^{*}$ satisfying $Y_{0} \sim[r-1] X^{*}$ and $Y_{0} \nsim X^{*}$. This contradicts the statement of Claim, and hence $c^{*}\left(X^{*}\right)=\lambda$ holds, as required.

We now prove Claim.

Proof of Claim. Take any $Y_{0} \in \mathcal{F}^{*}$ with $Y_{0} \sim_{[r-1]} X^{*}$. By $c_{p_{i} r}\left(X_{i}\right)>c^{*}\left(X^{*}\right)(i \in[k])$ and Lemma 3.8, for every $i \in[k]$ there is $Y \in \mathcal{F}^{*}$ with $Y \sim_{p_{i} r} X_{i}$ and $Y \nsim X^{*}$. Take $Y \in \mathcal{F}^{*}$ satisfying $Y \nsim X^{*}$ with $\left\{i \in[k] \mid Y \sim_{p_{i} r} X_{i}\right\}$ maximal among elements $Y^{\prime} \in \mathcal{F}^{*}$ satisfying $Y^{\prime} \nsim X^{*}$. Let $I:=\left\{i \in[k] \mid Y \sim_{p_{i} r} X_{i}\right\}(\neq \emptyset)$. By the maximality of $\left\langle X_{0}\right\rangle$ and $Y \nsim X^{*}$, we have $[k] \backslash I \neq \emptyset$; otherwise $\langle Y\rangle \cap A_{[r-1]} \supsetneq\left\langle X_{0}\right\rangle$, contradicting the maximality of $\left\langle X_{0}\right\rangle$.

Choose an arbitrary $j \in[k] \backslash I$. Then there is $Y_{j} \in \mathcal{F}^{*}$ with $Y_{j} \sim_{p_{j} r} X_{j}$ and $Y_{j} \nsim X^{*}$. Furthermore, by the maximality of $I$, there is $i \in I$ such that $Y_{j} \nsim_{p_{i} r} X$. Hence $Y_{j} \nsim_{p_{i}} Y \sim_{p_{i}} X^{*}$ holds. In the following, we denote $Y$ by $Y_{i}$.

Since $Y_{i}, Y_{j}, Y_{0} \in \mathcal{F}^{*}$ and $\mathcal{F}^{*}$ is laminarizable, so is $\left\{Y_{i}, Y_{j}, Y_{0}\right\}$. Hence, by executing appropriate transformations for $\left\{Y_{i}, Y_{j}, Y_{0}\right\}$, we can make it laminar. We also denote the resulting laminar family by $\left\{Y_{i}, Y_{j}, Y_{0}\right\}$. We can assume $Y_{i} \cap A_{p_{i}}=Y_{0} \cap A_{p_{i}}(\neq \emptyset)$ and $Y_{j} \cap A_{p_{j}}=Y_{0} \cap A_{p_{j}}(\neq \emptyset)$. Indeed, $Y_{i} \cap A_{p_{i}} \neq Y_{0} \cap A_{p_{i}}$ means $\left([n] \backslash Y_{i}\right) \cap A_{p_{i}}=Y_{0} \cap A_{p_{i}}$. By the laminarity of $Y_{i}$ and $Y_{0}$, we have $Y_{i} \cap Y_{0}=\emptyset$. Hence $\left\{[n] \backslash Y_{i}, Y_{0}\right\}$ is also laminar. Furthermore, note $Y_{i} \cap A_{r}=Y_{j} \cap A_{r}(\neq \emptyset)$ by $Y_{i} \sim_{p_{i} r} X_{i}$ and $Y_{j} \sim p_{p_{j} r} X_{j}$.

By $Y_{0} \sim_{p_{i} p_{j}} X^{*} \nsim_{p_{i} p_{j}} Y_{i}$ and laminarity, it holds that $Y_{0} \cap A_{p_{j}} \supsetneq Y_{i} \cap A_{p_{j}}$ or $Y_{0} \cap A_{p_{j}} \subsetneq Y_{i} \cap A_{p_{j}}$. Assume $Y_{0} \cap A_{p_{j}}=Y_{j} \cap A_{p_{j}} \subsetneq Y_{i} \cap A_{p_{j}}$ (the argument for the other case is similar). Hence, by $Y_{0} \cap Y_{i} \neq \emptyset$ and $Y_{j} \cap Y_{i} \neq \emptyset$, we have $Y_{0} \subsetneq Y_{i} \supsetneq Y_{j}$. By $Y_{j} \nsim_{p_{i}} Y_{i} \sim_{p_{i}} X$ and $Y_{i} \supsetneq Y_{j}$, we have $Y_{0} \cap A_{p_{i}}=Y_{i} \cap A_{p_{i}} \supsetneq Y_{j} \cap A_{p_{i}}$. Hence $Y_{i} \supsetneq Y_{0} \supsetneq Y_{j}$ holds. By $Y_{i} \cap A_{r}=Y_{j} \cap A_{r}$, it holds that $Y_{i} \cap A_{r}=Y_{j} \cap A_{r}=Y_{0} \cap A_{r}$. This means $Y_{0} \sim X^{*}$.

(ii). By Lemma 3.8, it holds that

$$
\begin{aligned}
c^{\prime}\left(X_{0}\right) & =\sum\left\{c^{*}(Y) \mid Y \in \mathcal{F}^{*}, Y \sim_{[r-1]} X_{0}\right\} \\
& =c^{*}\left(X_{0}\right)+\sum\left\{c^{*}(Y) \mid Y \in \mathcal{F}^{*},\langle Y\rangle \supseteq A_{r}, X_{0} \sim_{[r-1]} Y\right\} .
\end{aligned}
$$


Here the second term must be zero. Otherwise, by Lemma 3.8, we would have found $X_{1}, X_{2}, \ldots, X_{k}$ in Step 2. Therefore $c^{\prime}\left(X_{0}\right)=c^{*}\left(X_{0}\right)$ holds. Thus we obtain $c\left(X_{0}\right)=c^{\prime}\left(X_{0}\right)=c^{*}\left(X_{0}\right)$.

(iii). We can show $c(X)=c_{p_{i} k}(X)=c^{*}(X)$ for any $i \in[k]$ and $X \in \mathcal{L}_{p_{i} k}$ by a similar argument as for (ii).

(3). Note that $\left|\mathcal{F}^{\prime}\right|=O\left(\left|A_{[r-1]}\right|\right)$ and $\left|\mathcal{L}_{p r}\right|=O\left(\left|A_{p r}\right|\right)$ for any $p \in[r-1]$. By the assumption $\left|A_{r}\right| \leq$ $\min \left\{\left|A_{1}\right|,\left|A_{2}\right|, \ldots,\left|A_{r-1}\right|\right\}$, it holds that $r\left|A_{r}\right|=O(n)$. Step 1 can be done in $O\left(\sum_{p \in[r-1]}\left(\left|A_{p}\right|+\right.\right.$ $\left.\left.\left|A_{r}\right|\right)^{2}\right)=O\left(\sum_{p \in[r-1]}\left|A_{p}\right|^{2}\right)=O\left(n^{2}\right)$ time by Proposition 3.4. In Step 2, we first need to sort the elements in $\mathcal{F}^{\prime}$ with respect to set-inclusion ordering in $O\left(\left|A_{[r-1]}\right| \log \left|A_{[r-1]}\right|\right)=O(n \log n)$ time (this is done only once). In each iteration, we search for $\left\{X_{1}, X_{2}, \ldots, X_{k}\right\}$ satisfying the conditions described in Step 2. This can be done in $O\left(\left|\cup_{p} \mathcal{L}_{p r}\right|\right)=O\left(n+r\left|A_{r}\right|\right)=O(n)$ time by using the structure of $\mathcal{L}_{p_{i} r}$ as follows.

We first construct $\mathcal{F}_{i}$ from $\mathcal{L}_{p_{i} r}$ as $\mathcal{F}_{i}:=\mathcal{G}_{i} \cup \overline{\mathcal{G}_{i}}$ for all $i \in[k]$ in $O\left(\left|\cup_{p} \mathcal{L}_{p r}\right|\right)=O(n)$ time, where

$$
\begin{aligned}
& \mathcal{G}_{i}:=\left\{X_{i} \cap A_{r} \mid X_{i} \in \mathcal{L}_{p_{i} r}, X_{i} \cap A_{p_{i}}=X_{0} \cap A_{p_{i}}\right\}, \\
& \overline{\mathcal{G}_{i}}:=\left\{A_{r} \backslash X_{i} \mid X_{i} \in \mathcal{L}_{p_{i} r}, A_{p_{i}} \backslash X_{i}=X_{0} \cap A_{p_{i}}\right\} .
\end{aligned}
$$

Note that $F \cup\left(X_{0} \cap A_{p_{i}}\right) \in \mathcal{L}_{p_{i} r}$ if $F \in \mathcal{G}_{i}$ and $\left(A_{r} \backslash F\right) \cup\left(A_{p_{i}} \backslash X_{0}\right) \in \mathcal{L}_{p_{i} r}$ if $F \in \overline{\mathcal{G}_{i}}$. We can easily see that there exists $\left\{X_{1}, X_{2}, \ldots, X_{k}\right\}$ satisfying the conditions in Step 2 if and only if $\bigcap_{i \in[k]} \mathcal{F}_{i} \neq \emptyset$. By the laminarity of $\mathcal{L}_{p_{i} r}, \mathcal{F}_{i}$ is a chain, and can be represented as $\mathcal{F}_{i}=\left\{F_{i}^{1}, F_{i}^{2}, \ldots, F_{i}^{q_{i}}\right\}$ for $i \in[k]$, where $F_{i}^{1} \supsetneq F_{i}^{2} \supsetneq \cdots \supsetneq F_{i}^{q_{i}}$ (this chain can be obtained while constructing $\mathcal{L}_{p_{i} r}$ in Algorithm 1). If $\bigcap_{i \in[k]} \mathcal{F}_{i} \neq \emptyset$, we can obtain $F \in \bigcap_{i \in[k]} \mathcal{F}_{i}$ in $O\left(\sum_{i}\left|\mathcal{F}_{i}\right|\right)=O(n)$ time. Indeed, take the maximal elements $F_{1}^{1}, F_{2}^{1}, \ldots, F_{k}^{1}$ in $\mathcal{F}_{1}, \mathcal{F}_{2}, \ldots, \mathcal{F}_{k}$, respectively. If all $i$ satisfy $F_{i}^{1}=\bigcap_{j} F_{j}^{1}$, then output $\bigcap_{j} F_{j}^{1}$. Otherwise, for each $i$ with $F_{i}^{1} \supsetneq \bigcap_{j} F_{j}^{1}$, update $\mathcal{F}_{i} \leftarrow \mathcal{F}_{i} \backslash\left\{F_{i}^{1}\right\}$, and do the same thing. By repeating this procedure, we can verify $\bigcap_{i \in[k]} \mathcal{F}_{i}=\emptyset$ or obtain $F \in \bigcap_{i \in[k]} \mathcal{F}_{i}$. From this $F$ in $\bigcap_{i \in[k]} \mathcal{F}_{i}$, we can easily construct the desired $X_{i}$ as

$$
X_{i}= \begin{cases}F \cup\left(X_{0} \cap A_{p_{i}}\right) & \text { if } F \in \mathcal{G}_{i}, \\ \left(A_{r} \backslash F\right) \cup\left(A_{p_{i}} \backslash X_{0}\right) & \text { if } F \in \overline{\mathcal{G}_{i}}\end{cases}
$$

for each $i \in[k]$. Thus we can find $\left\{X_{1}, X_{2}, \ldots, X_{k}\right\}$ satisfying the conditions in Step 2 in $O(n)$ time.

Furthermore we can calculate $\min \left\{c^{\prime}\left(X_{0}\right), c_{p_{1} r}\left(X_{1}\right), c_{p_{2} r}\left(X_{2}\right), \ldots, c_{p_{k} r}\left(X_{k}\right)\right\}$ in $O(k)=O(n)$ time. Since $\left|\mathcal{F}^{\prime}\right|+\left|\bigcup_{p} \mathcal{L}_{p r}\right|$ decreases at least by one in each iteration in Step 2, the number of iterations in Step 2 is bounded by $O\left(\left|\mathcal{F}^{\prime}\right|+\left|\bigcup_{p} \mathcal{L}_{p r}\right|\right)=O(n)$. Hence Step 2 can be done in $O\left(n^{2}\right)$ time.

Step 3 can be done in $O\left(\left|\cup_{p} \mathcal{L}_{p r}\right|+n\right)=O(n)$ time. Hence the running-time of Algorithm 2 is bounded by $O\left(n^{2}\right)$.

Our proposed algorithm for Decomposition can be summarized as follows.

\section{Algorithm 3 (for Decomposition):}

Step 0: Rename $A_{1}, A_{2}, \ldots, A_{r}$ so as to satisfy $\left|A_{1}\right| \geq\left|A_{2}\right| \geq \cdots \geq\left|A_{r}\right|$.

Step 1: Execute Algorithm 1 for the restriction $f_{12}$. If Algorithm 1 returns " $f_{12}$ is not QR-M $2_{2}$ convex," then output " $f$ is not QR-M $\mathrm{M}_{2}$-convex" and stop. Otherwise, obtain $\mathcal{L}_{12}$ and $c_{12}$.

Step 2: For $t=3, \ldots, r$, execute Algorithm 2 for $\left(\mathcal{F}_{[t-1]}, c_{[t-1]}\right)$, where $\mathcal{F}_{[2]}=\mathcal{L}_{12}$ and $c_{[2]}=c_{12}$. If Algorithm 2 returns " $f_{[t]}$ is not QR-M $\mathrm{M}_{2}$-convex," output " $f$ is not $\mathrm{QR}-\mathrm{M}_{2}$-convex" and stop. Otherwise, obtain $\left(\mathcal{F}_{[t]}, c_{[t]}\right)$.

Step 3: Output $\left(\mathcal{F}_{[r]}, c_{[r]}\right)$.

Theorem 3.9. Algorithm 3 solves Decomposition in $O\left(r n^{2}\right)$ time. 
Proof. Step 0 can be done in $O(r \log r)$ time. Since the running-time of Algorithm 2 for $r^{\prime}$ is bounded by $O\left(\left|A_{\left[r^{\prime}+1\right]}\right|^{2}\right)=O\left(n^{2}\right)$ by Proposition 3.7 (3), the running-time of Algorithm 3 is bounded by $O\left(r n^{2}\right)$.

The validity of Algorithm 3 can be proved as follows. Suppose that Algorithm 3 stops at Step 1 or Step 2. By Proposition 3.4 and Proposition 3.7 (2), $f$ is not $Q R-M_{2}$-convex. Hence Algorithm 3 works correctly.

Suppose that Algorithm 3 reaches Step 3. Since $f_{[2]} \simeq_{[2]} \sum_{X \in \mathcal{F}_{[2]}} c_{[2]}(X) \ell_{X}$ by Proposition 3.4, we obtain $f_{[t]} \simeq_{[t]} \sum_{X \in \mathcal{F}_{[t]}} c_{[t]}(X) \ell_{X}$ for all $t=3, \ldots, r$ by Proposition 3.7 (1), where $\simeq_{Q}$ denotes the $\mathcal{A}_{Q}$-linear equivalence and this notation is used for $Q=[2], \ldots,[r]$ here. Thus we have $f \simeq \sum_{X \in \mathcal{F}_{[r]}} c_{[r]}(X) \ell_{X}$ holds. Furthermore, if $f$ is $\mathrm{QR}-\mathrm{M}_{2}$-convex, then $\mathcal{F}_{[2]}\left(=\mathcal{L}_{12}\right)$ is laminarizable by Lemma 3.1 and Proposition 3.4. Hence $\mathcal{F}_{[3]}, \ldots, \mathcal{F}_{[r]}$ are laminarizable by Proposition 3.7 (2). Thus Algorithm 3 works correctly.

\section{ALGORITHM FOR LAMINARIZATION}

For a VCSP-quadratic function $f$ of type $\mathcal{A}=\left\{A_{1}, A_{2}, \ldots, A_{r}\right\}$, suppose that we have obtained a non-redundant $\mathcal{A}$-cut family $\mathcal{F}$ by solving Decomposition. The next step for solving Testing QuAdRATIC $\mathrm{M}_{2}$-REPRESENTABILITy is to check for the laminarizability of $\mathcal{F}$.

Recall that a pair $X, Y \subseteq[n]$ is said to be crossing if $X \cap Y,[n] \backslash(X \cup Y), X \backslash Y$, and $Y \backslash X$ are all nonempty. An $\mathcal{A}$-cut family $\mathcal{G}$ is said to be cross-free if there is no crossing pair in $\mathcal{G}$. From a cross-free $\mathcal{A}$-cut family $\mathcal{G}$, we can easily construct a laminar $\mathcal{A}$-cut family $\mathcal{A}$-equivalent to $\mathcal{G}$ by switching $X \mapsto[n] \backslash X$ for appropriate $X \in \mathcal{G}$ (see e.g., [23, Section 2.2]); this can be done in $O(|\mathcal{G}|)$ time. Furthermore, if $\mathcal{F}$ is laminarizable, then we can always construct a cross-free family $\mathcal{A}$-equivalent to $\mathcal{F}$ without using transformation $X \mapsto[n] \backslash X$. Thus our goal is to construct a cross-free family $\mathcal{A}$-equivalent to the input family $\mathcal{F}$ by repeating appropriate transformations for $X \in \mathcal{F}$ as $X \mapsto X \cup A_{p}$ or $X \mapsto X \backslash A_{p}$ with some $A_{p}$ satisfying $\langle X\rangle \cap A_{p}=\emptyset$. Recall that $\langle X\rangle$ denote the cutting support of $X$ defined in (2.3).

In this section, we devise a polynomial-time algorithm for constructing a desired cross-free family. Our algorithm makes use of weaker notions of cross-freeness, called 2- and 3-local cross-freeness. The existence of a cross-free family is characterized by the existence of a 2-locally cross-free family (Section 4.2). The existence of a 2-locally cross-free family can be checked easily by solving a 2-SAT problem. If a 2-locally cross-free family exists, then a 3-locally cross-free family also exists, and can be constructed in polynomial time (Section 4.4). From a 3-locally cross-free family, we can construct a desired cross-free family in polynomial time via the uncrossing operation (Section 4.3). Thus we solve LAMINARIZATION.

\subsection{Preliminaries}

We use the following notations and terminologies. For $X \in \mathcal{F}$, let $\bar{X}:=\langle X\rangle \backslash X$; note $X \sim \bar{X}$ by Lemma 2.2 (2). For $\mathcal{A}$-cuts $X, Y, Z$, we define $\langle X Y\rangle:=\langle X\rangle \cap\langle Y\rangle$ and $\langle X Y Z\rangle:=\langle X\rangle \cap\langle Y\rangle \cap\langle Z\rangle$. For $X \in \mathcal{F}$ and $Q \subseteq[r]$ with $A_{Q} \subseteq\langle X\rangle$, the partition line of $X$ on $A_{Q}$ is a bipartition $\left\{X \cap A_{Q}, \bar{X} \cap A_{Q}\right\}$ of $A_{Q}$. For $A \subseteq[n]$, if $X \cap A \subseteq Y \cap A$ holds, we say $X \subseteq Y$ on $A$.

Without loss of generality, we can assume the following:

- $|\mathcal{F}|$ is at most $2 n$.

- For distinct $X, Y \in \mathcal{F}$ with $\langle X Y\rangle \neq \emptyset$, one of $X \subseteq Y, X \subseteq \bar{Y}, X \supseteq Y$, and $X \supseteq \bar{Y}$ holds on $\langle X Y\rangle$.

- For all distinct $X, Y \in \mathcal{F}$, both $\langle X\rangle \backslash\langle Y\rangle$ and $\langle Y\rangle \backslash\langle X\rangle$ are nonempty.

If the first or the second condition fails, then $\mathcal{F}$ is not laminarizable. The third condition is satisfied by the following preprocessing. For each $X \in \mathcal{F}$, we add a new set $A_{X}$ with $\left|A_{X}\right|=2$ to the ground 
set $[n]$ and to the partition $\mathcal{A}$ of $[n]$; the ground set will be $[n] \cup \cup_{X \in \mathcal{F}} A_{X}$ and the partition will be $\mathcal{A} \cup\left\{A_{X} \mid X \in \mathcal{F}\right\}$. Define $X_{+}:=X \cup\{x\}$, where $x$ is one of the two elements of $A_{X}$ and $\mathcal{F}_{+}:=\left\{X_{+} \mid X \in \mathcal{F}\right\}$. Note $\left\langle X_{+}\right\rangle=\langle X\rangle \cup A_{X}$ and $\left\langle X_{+}\right\rangle \backslash\left\langle Y_{+}\right\rangle \neq \emptyset$ for all $X_{+}, Y_{+} \in \mathcal{F}_{+}$. Then it is easily seen that there exists a cross-free family $\mathcal{L}$ with $\mathcal{L} \sim \mathcal{F}$ if and only if there exists a cross-free family $\mathcal{L}_{+}$with $\mathcal{L}_{+} \sim \mathcal{F}_{+}$. Furthermore we can construct the cross-free family $\mathcal{L}$ from $\mathcal{L}_{+}$by restricting $\mathcal{L}_{+}$to $[n]$, that is, $\mathcal{L}=\left\{L \cap[n] \mid L \in \mathcal{L}_{+}\right\}$.

\subsection{2-local cross-freeness}

For $A \subseteq[n]$, a pair $X, Y \subseteq[n]$ is said to be crossing on $A$ if $(X \cap Y) \cap A, A \backslash(X \cup Y),(X \backslash Y) \cap A$, and $(Y \backslash X) \cap A$ are all nonempty. An $\mathcal{A}$-cut family $\mathcal{G}$ is said to be cross-free on $A$ if there is no crossing pair on $A$ in $\mathcal{G}$. An $\mathcal{A}$-cut family $\mathcal{G}$ is called 2-locally cross-free if no $X, Y \in \mathcal{G}$ are crossing on $\langle X\rangle \cup\langle Y\rangle$. A cross-free family is 2-locally cross-free. We denote the ordered pair $(X, Y)$ by $X Y$.

Our goal of this subsection is to construct a 2-locally cross-free family $\mathcal{F}^{*}$ that is $\mathcal{A}$-equivalent to the input $\mathcal{F}$ (if it exists). Such $\mathcal{F}^{*}$ consists of $X^{*}$ that is obtained from each $X \in \mathcal{F}$ by adding or deleting some $A_{p}$ not intersecting with the cutting support $\langle X\rangle$ of $X$, i.e., $X^{*}=\left(X \backslash \cup_{p \in I} A_{p}\right) \cup$ $\left(\cup_{p \in J} A_{p}\right)$ for some $I, J \subseteq[r]$, where $A_{p} \cap\langle X\rangle=\emptyset$ for all $p \in I \cup J$. By the 2-local cross-freeness, for each ordered pair $X Y$ of members $X, Y$ in $\mathcal{F}$, either one of the following holds:

$(X Y: 0) X^{*}$ contains no $A_{p}$ contained in $\langle Y\rangle \backslash\langle X\rangle$, i.e., $X^{*} \cap(\langle Y\rangle \backslash\langle X\rangle)=\emptyset$.

$(X Y: 1) X^{*}$ contains every $A_{p}$ contained in $\langle Y\rangle \backslash\langle X\rangle$, i.e., $X^{*} \supseteq\langle Y\rangle \backslash\langle X\rangle$.

It turns out that a desired 2-locally cross-free family is obtained by specifying $(X Y: 0)$ or $(X Y: 1)$, called the label of $X Y$, for all ordered pairs $X Y$. We observe that the labels satisfy the following properties:

- Suppose that $\langle X Y\rangle \neq \emptyset$ and the partition lines of $X, Y$ on $\langle X Y\rangle$ are different. Then the labels of $X Y$ and $Y X$ are determined uniquely by their mutual configuration. For example, if $X \subsetneq Y$ on $\langle X Y\rangle$, then we have $X^{*} \subseteq Y^{*}$ on $\langle X\rangle \cup\langle Y\rangle$, namely, $(X Y: 0)$ and $(Y X: 1)$ hold. Also if $X \subsetneq \bar{Y}$ on $\langle X Y\rangle$, then we have $X^{*} \cap Y^{*}=\emptyset$ on $\langle X\rangle \cup\langle Y\rangle ;(X Y: 0)$ and $(Y X: 0)$ hold. Similarly for the remaining cases, $X \supsetneq Y$ or $X \supsetneq \bar{Y}$ on $\langle X Y\rangle$.

- Suppose that $\langle X Y\rangle \neq \emptyset$ and the partition lines of $X, Y$ on $\langle X Y\rangle$ are the same. In this case, the labels of $X Y$ and $Y X$ are not uniquely determined. If the label of $Y X$ is given, then the label of $X Y$ is determined according to the mutual configuration of $X$ and $Y$ on $\langle X Y\rangle$. For example, suppose that we have $X=Y$ on $\langle X Y\rangle$. Then $(Y X: 1)$ implies $(X Y: 0)$ and vice versa.

- Suppose that $X, Y, Z \in \mathcal{F}$ satisfy $\langle Y Z\rangle \backslash\langle X\rangle \neq \emptyset$. Then the labels of $X Y$ and $X Z$ must be the same. Indeed, if $(X Y: 1)$ holds, i.e., $X^{*} \supseteq\langle Y\rangle \backslash\langle X\rangle$, then $X^{*} \cap(\langle Z\rangle \backslash\langle X\rangle)$ are nonempty on $\langle X\rangle \cup\langle Z\rangle$. This implies that $(X Z: 1)$ holds.

An $L C$-labeling $s$ for $\mathcal{F}$ is a function on the set of ordered pairs of distinct members in $\mathcal{F}$ satisfying the above properties, i.e.,

$$
\begin{aligned}
& (s(X Y), s(Y X))= \begin{cases}(0,0) & \text { if } X \subsetneq \bar{Y} \text { on }\langle X Y\rangle, \\
(0,1) & \text { if } X \subsetneq Y \text { on }\langle X Y\rangle, \\
1,0) & \text { if } X \supsetneq Y \text { on }\langle X Y\rangle, \\
(1,1) & \text { if } X \supsetneq \bar{Y} \text { on }\langle X Y\rangle,\end{cases} \\
& s(X Y)= \begin{cases}s(Y X) & \text { if } X \subseteq \bar{Y} \text { or } X \supseteq \bar{Y} \text { on }\langle X Y\rangle, \\
1-s(Y X) & \text { if } X \subseteq Y \text { or } X \supseteq Y \text { on }\langle X Y\rangle,\end{cases} \\
& s(X Y)=s(X Z) \text { if }\langle Y Z\rangle \backslash\langle X\rangle \neq \emptyset,
\end{aligned}
$$




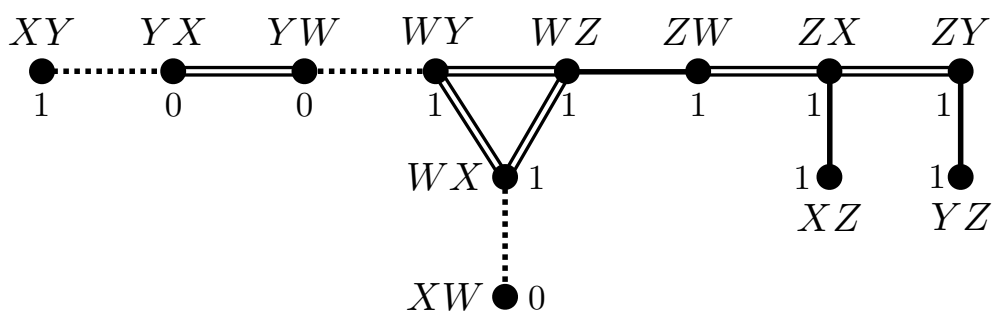

Fig. 5. The LC-graph $G(\mathcal{F})$ for $\mathcal{F}=\{X, Y, Z, W\}$ in Example 4.2, where the edges denoted by double lines are prefixed edges, and the others are swapped edges. Flipping and non-flipping swapped edges are denoted by dotted and solid line, respectively. The numbers $0 / 1$ at the nodes denote the LC-labeling $s$ in case of setting $s(X Y)=1$.

where (4.1) and (4.2) apply only when $\langle X Y\rangle \neq \emptyset$. Here LC stands for Local Cross-freeness.

From the definition, it is obvious that any 2-locally cross-free family $\mathcal{F}^{*}$ that is $\mathcal{A}$-equivalent to $\mathcal{F}$ (without taking complements) gives rise to an LC-labeling $s$ for $\mathcal{F}$. Indeed, define $s(X Y):=0$ if $X^{*}$ is in case $(X Y: 0)$ and $s(X Y):=1$ if $X^{*}$ is in case $(X Y: 1)$. The converse is also possible. Let $s$ be an LC-labeling for $\mathcal{F}$. Consider the following procedure for each $X \in \mathcal{F}$ : For each $A_{p} \in \mathcal{A}$ with $A_{p} \subseteq\langle Y\rangle \backslash\langle X\rangle$ for some $Y$, if $s(X Y)=1$, then add $A_{p}$ to $X$, and if $s(X Y)=0$, then delete $A_{p}$ from $X$. Let $X^{s}$ denote the resulting set. Thanks to the condition (4.3), this procedure is independent of the choice of $Y$ and is well-defined. Accordingly, define $\mathcal{F}^{s}$ by

$$
\mathcal{F}^{s}:=\left\{X^{s} \mid X \in \mathcal{F}\right\} .
$$

Then $\mathcal{F}^{s}$ is indeed 2-locally cross-free. To see this, it suffices to consider $X, Y$ with $\langle X Y\rangle \neq \emptyset$. By (4.1) and (4.2), it holds $X^{s} \subseteq Y^{s}, X^{s} \supseteq Y^{s}, X^{s} \cap Y^{s}=\emptyset$, or $(\langle X\rangle \cup\langle Y\rangle) \backslash\left(X^{s} \cup Y^{s}\right)=\emptyset$ on $\langle X\rangle \cup\langle Y\rangle$. Thus the following holds.

Proposition 4.1. There exists a 2-locally cross-free family $\mathcal{A}$-equivalent to $\mathcal{F}$ if and only if there exists an LC-labeling s for $\mathcal{F}$. To be specific, $\mathcal{F}^{s}$ is a 2-locally cross-free family $\mathcal{A}$-equivalent to $\mathcal{F}$.

In order to finding an LC-labeling in a greedy fashion, we introduce the $L C$-graph, which is also utilized for constructing a 3-locally cross-free family $\mathcal{A}$-equivalent to $\mathcal{F}$ in Section 4.4. The $L C$-graph $G(\mathcal{F})=\left(V(\mathcal{F}), E_{\mathrm{s}} \cup E_{\mathrm{p}}\right)$ of the input $\mathcal{F}$ is defined by

$$
\begin{aligned}
V(\mathcal{F}) & :=\{X Y \mid X, Y \in \mathcal{F}, X \neq Y\}, \\
E_{\mathrm{s}} & :=\{\{X Y, Y X\} \mid\langle X Y\rangle \neq \emptyset\}, \\
E_{\mathrm{p}} & :=\{\{X Y, X Z\} \mid Y \neq Z,\langle Y Z\rangle \backslash\langle X\rangle \neq \emptyset\} .
\end{aligned}
$$

Note that the structure of LC-graph depends only on the family $\{\langle X\rangle \mid X \in \mathcal{F}\}$ of cutting supports. We call an edge $e \in E_{\mathrm{s}}$ a swapped edge, which corresponds to (4.1) and (4.2), and an edge $e \in E_{\mathrm{p}}$ a prefixed edge, which corresponds to (4.3). By the second assumption mentioned in Section 4.1, exactly two types of swapped edges $e=\{X Y, Y X\}$ can be distinguished; (i) $X \subseteq Y$ or $X \supseteq Y$ on $\langle X Y\rangle$ and (ii) $X \subseteq \bar{Y}$ or $X \supseteq \bar{Y}$ on $\langle X Y\rangle$. The former type of swapped edges will be called flipping (since $s(X Y)=1-s(Y X)$ ), and the latter type non-flipping (since $s(X Y)=s(Y X))$. See Figure 5 for an example of LC-graph.

An LC-labeling is nothing but a feasible solution for the 2-SAT problem defined by the constraints (4.1)-(4.3). Therefore we can check the existence of an LC-labeling $s$ greedily in $O\left(\mid E_{\mathrm{s}} \cup\right.$ $\left.E_{\mathrm{p}} \mid\right)=O\left(n^{4}\right)$ time. Node $X Y \in V(\mathcal{F})$ is said to be fixed if the value of an LC-labeling $s$ for $X Y$ is determined as (4.1), that is, if $\langle X Y\rangle \neq \emptyset$ and the partition lines of $X$ and $Y$ on $\langle X Y\rangle$ are different, and $X Y$ is said to be defined if the value of $s(X Y)$ has been defined. The algorithm is as follows. 
(1) For each fixed node $X Y$, define $s(X Y)$ according to (4.1).

(2) In each connected component of $G(\mathcal{F})$, execute a breadth-first search from a defined node $X Y$, and define $s(Z W)$ for all reached nodes $Z W$ according to (4.2) and (4.3). If a conflict in value assignment to $s(Z W)$ is detected during this process, output "there is no LC-labeling."

(3) If there is an undefined node, choose any undefined node $X Y$, and define $s(X Y)$ as 0 or 1 arbitrarily. Then go to 2 .

Example 4.2. We consider the family $\mathcal{F}$ obtained in Example 3.6. After applying the preprocessing to $\mathcal{F}$, it holds $\mathcal{F}=\{X, Y, Z, W\}$, where $X:=1357, Y:=135 a, Z:=24 b$, and $W:=37 c$ with the partition $\mathcal{A}=\left\{12,34,56,78, a a^{\prime}, b b^{\prime}, c c^{\prime}\right\}$ of the ground set $N:=12345678 a a^{\prime} b b^{\prime} c c^{\prime}$. The LC-graph $G(\mathcal{F})$ is illustrated in Figure 5.

We obtain an LC-labeling $s: V(\mathcal{F}) \rightarrow\{0,1\}$ by defining, for example, $s(X Y):=1$ as $s\left(X^{\prime} Y^{\prime}\right)=0$ for $X^{\prime} Y^{\prime} \in\{Y X, Y W, X W\}$ and $s\left(X^{\prime} Y^{\prime}\right)=1$ otherwise. Then $X^{s}=1357 a a^{\prime} b b^{\prime}, Y^{s}=135 a b b^{\prime}$, $Z^{s}=245678 a a^{\prime} b c c^{\prime}, W^{s}=123567 a a^{\prime} b b^{\prime} c$, and $\mathcal{F}^{s}$ is a cross-free family with $\mathcal{F}^{s} \sim \mathcal{F}$. Thus $\mathcal{F}^{\prime}:=\left\{X^{s}, Y^{s}, N \backslash Z^{s}, N \backslash W^{s}\right\}$ is a laminar family with $\mathcal{F}^{\prime} \sim \mathcal{F}$.

Recall that the original $\mathcal{F}$ is a family of subsets of 12345678 . Let $\mathcal{L}$ be the family of $\mathcal{F}^{\prime}$ restricted to 12345678 , i.e., $\mathcal{L}=\{1357,135,13,48\}$, which is the same one as the family inducing M-convex summand $f_{1}$ defined in (1.5); see also Figure 1.

\subsection{3-local cross-freeness}

An $\mathcal{A}$-cut family $\mathcal{G}$ is called 3-locally cross-free if $\mathcal{G}$ is 2-locally cross-free and $\{X, Y, Z\}$ is cross-free on the union of the cutting supports $\langle X\rangle \cup\langle Y\rangle \cup\langle Z\rangle$ for all $X, Y, Z \in \mathcal{G}$ that have a nonempty intersection of the cutting supports, i.e., $\langle X Y Z\rangle \neq \emptyset$. A cross-free family is 3-locally cross-free, and a 3-locally cross-free family is 2-locally cross-free, whereas the converse is not true (see Remark 4.5). We write $X \subseteq \subseteq^{*} Y$ to mean $X \subseteq Y$ on $\langle X\rangle \cup\langle Y\rangle$.

Our objective of this subsection is to give an algorithm for constructing a desired cross-free family from a 3 -locally cross-free family $\mathcal{A}$-equivalent to the input $\mathcal{F}$. The algorithm consists of repeated applications of an elementary operation that preserves 3-local cross-freeness. The operation is defined by (4.5) below, and is referred to as the uncrossing operation to $X, Y$. By the 2-local cross-freeness of $\mathcal{G}$, the two cases in (4.5) exhaust all possibilities for $X, Y \in \mathcal{G}$.

Proposition 4.3. Suppose that $\mathcal{G}$ is 3-locally cross-free. For $X, Y \in \mathcal{G}$, define

$$
\mathcal{G}^{\prime}:= \begin{cases}\mathcal{G} \backslash\{X, Y\} \cup\{X \cap Y, X \cup Y\} & \text { if } X \subseteq^{*} Y \text { or } Y \subseteq^{*} X, \\ \mathcal{G} \backslash\{X, Y\} \cup\{X \backslash Y, Y \backslash X\} & \text { if } X \subseteq^{*}[n] \backslash Y \text { or }[n] \backslash Y \subseteq^{*} X .\end{cases}
$$

Then $\mathcal{G}^{\prime}$ is a 3-locally cross-free family $\mathcal{A}$-equivalent to $\mathcal{G}$.

The proof of Proposition 4.3 is given at the end of this subsection.

Algorithm 4 (for constructing a cross-free family):

Input: A 3-locally cross-free family $\mathcal{G}$.

Step 1: While there is a crossing pair $X, Y$ in $\mathcal{G}$, apply the uncrossing operation to $X, Y$ and modify $\mathcal{G}$ accordingly.

Step 2: Output $\mathcal{G}$.

Proposition 4.4. Algorithm 4 runs in $O\left(n^{2}\right)$ time, and the output $\mathcal{G}$ is cross-free.

Proof. The number of crossing pairs in input $\mathcal{G}$ is at most $O\left(n^{2}\right)$ (since $|\mathcal{G}|=O(n)$ ). Take any $\{X, Y\} \subseteq \mathcal{G}$ which is crossing. Since the replacement $X \mapsto[n] \backslash X$ or $Y \mapsto[n] \backslash Y$ does not change the (non-)cross-freeness of $\{X, Y\},\{X, Z\}$, and $\{Y, Z\}$ for $Z \in \mathcal{G}$, we can assume $X \subseteq^{*} Y$ or $Y \subseteq^{*} X$ by appropriate replacement. Let $\mathcal{G}^{\prime}$ be the family resulting from the uncrossing operation 
on $X, Y$. Then it is easily verified that, for any $Z \in \mathcal{G} \backslash\{X, Y\}$, the number of crossing pairs in $\{\{X \cap Y, Z\},\{X \cup Y, Z\}\}$ is at most that in $\{\{X, Z\},\{Y, Z\}\}$. Since $\{X \cap Y, X \cup Y\}$ is not crossing, the number of crossing pairs decreases at least by one. Furthermore, by Proposition 4.3, $\mathcal{G}^{\prime}$ is also a 3-locally cross-free family $\mathcal{A}$-equivalent to $\mathcal{F}$. Eventually, we arrive at a cross-free family $\mathcal{A}$-equivalent to $\mathcal{F}$. The above process involves at most $O\left(n^{2}\right)$ uncrossing operations.

Remark 4.5. It is worth mentioning that the uncrossing operation does not preserve 2-local cross-freeness. For example, we define $X:=1356, Y:=1347$, and $Z:=1578$ with a partition $\{12,34,56,78\}$. Note that $\{X, Y, Z\}$ is not 3-locally cross-free but 2-locally cross-free.

We consider to execute the uncrossing operation to $X, Y$. Then the resulting family is $\{X \cap Y, X \cup$ $Y, Z\}$. Since $X \cap Y=13$ and $Z=1578,\{X \cap Y, Z\}$ is crossing on $\langle X \cap Y\rangle \cup\langle Z\rangle=123456$.

The rest of this subsection is devoted to the proof of Proposition 4.3. We first note the following facts, which are also used in the proof of Proposition 4.11 in Section 4.4.

Lемма 4.6. Let $\mathcal{G}$ be a 2-locally cross-free family. A triple $\{X, Y, Z\} \subseteq \mathcal{G}$ is cross-free on $\langle X\rangle \cup$ $\langle Y\rangle \cup\langle Z\rangle$ if one of the following conditions holds:

(1) $\langle X Y\rangle \neq \emptyset$, and $\{X, Y\}$ is cross-free on $\langle X\rangle \cup\langle Y\rangle \cup\langle Z\rangle$.

(2) $\langle X Y\rangle \nsubseteq\langle Z\rangle$, and $\langle X Z\rangle$ or $\langle Y Z\rangle$ is nonempty.

(3) The partition lines of $X, Y, Z$ on $\langle X Y Z\rangle$ are not the same.

(4) $\langle X Y\rangle=\langle Z Y\rangle \neq \emptyset$, and there is a path $\left(X Y, X Y_{1}, \ldots, X Y_{k}\right)$ in $G(G)$ such that $\left\{X, Y_{k}, Z\right\}$ is cross-free on $\langle X\rangle \cup\left\langle Y_{k}\right\rangle \cup\langle Z\rangle$.

Proof. Let $S:=\langle X\rangle \cup\langle Y\rangle \cup\langle Z\rangle$. Note that $\{X, Y\}$ is 2-locally cross-free if and only if so is $\{[n] \backslash X, Y\}$. Hence, by appropriate replacement $X \mapsto[n] \backslash X$ and/or $Y \mapsto[n] \backslash Y$, we can assume $X \subseteq^{*} Y$; we often use such replacement in this proof.

(1). By symmetry, it suffices to show that $\{X, Z\}$ is cross-free on $S$. We assume $X \subseteq \subseteq^{*} Z$ (the argument for the case of $Z \subseteq^{*} X$ is similar). There are two cases: (i) $\langle X Y\rangle \backslash\langle Z\rangle \neq \emptyset$ and (ii) $(\emptyset \neq)\langle X Y\rangle \subseteq\langle Z\rangle$. Note that $X \subseteq \subseteq^{*} Z$ implies $Z \supseteq\langle X\rangle \backslash\langle Z\rangle$ and $X \cap(\langle Z\rangle \backslash\langle X\rangle)=\emptyset$.

(i). By the 2-local cross-freeness of $\{Y, Z\}$ and $\langle X Y\rangle \backslash\langle Z\rangle \neq \emptyset, Z \supseteq\langle X\rangle \backslash\langle Z\rangle$ implies $Z \supseteq\langle Y\rangle \backslash\langle Z\rangle$, and hence $Z \supseteq(\langle X\rangle \cup\langle Y\rangle) \backslash\langle Z\rangle$ holds. Thus $X \subseteq Z$ holds on $S$.

(ii). We can assume $Y \subseteq X$ or $X \subseteq Y$ on $S$. Then, by $\emptyset \neq\langle X Y\rangle=\langle X Y Z\rangle$ and the 2-local cross-freeness of $\{Y, Z\}$, we have $Y \subseteq^{*} Z$ or $Z \subseteq^{*} Y$. If $Y \subseteq^{*} Z$, then $Z \supseteq\langle Y\rangle \backslash\langle Z\rangle$ holds on $S$. Hence $X \subseteq Z$ holds on $S$. If $Z \subseteq^{*} Y$, then $X \subseteq Y$ must hold on $S$ by $X \subseteq^{*} Z$. This means $X \subseteq^{*} Y$, i.e., $X \cap(\langle Y\rangle \backslash\langle X\rangle)=\emptyset$. Hence $X \subseteq Z$ holds on $S$.

(2). We can assume $X \subseteq^{*} Y$ and $\langle X Z\rangle \neq \emptyset$. By $\langle X Y\rangle \nsubseteq\langle Z\rangle$ and $\langle X Z\rangle \neq \emptyset$, there are two cases: (i) $\langle X Z\rangle \nsubseteq\langle Y\rangle$ or (ii) $(\emptyset \neq)\langle X Z\rangle \subsetneq\langle X Y\rangle$.

(i). $X \subseteq^{*} Y$ implies $Y \supseteq\langle X\rangle \backslash\langle Y\rangle$. By $\langle X Z\rangle \nsubseteq\langle Y\rangle$, we have $Y \cap(\langle Z\rangle \backslash\langle Y\rangle) \neq \emptyset$. Hence, by the 2-local cross-freeness of $\{Y, Z\}, Y$ must contain $\langle Z\rangle \backslash\langle Y\rangle$. Therefore, it holds that $X \subseteq Y$ on $S$; then we use (1) (note $\langle X Y\rangle \neq \emptyset)$.

(ii). We assume $X \subseteq^{*} Z$ by the 2-local cross-freeness of $\{X, Z\}$ (the argument for the case of $Z \subseteq^{*} X$ is similar). This implies $Z \supseteq\langle X\rangle \backslash\langle Z\rangle$. By $\emptyset \neq\langle X Z\rangle \subsetneq\langle X Y\rangle$, we have $Z \cap(\langle Y\rangle \backslash\langle Z\rangle) \neq \emptyset$. Hence, by the 2-local cross-freeness of $\{Y, Z\}, Z$ must contain $\langle Y\rangle \backslash\langle Z\rangle$. Therefore, it holds that $X \subseteq Z$ on $S$; then we use (1).

(3). Note that $\langle X Y\rangle,\langle Y Z\rangle$, and $\langle Z X\rangle$ are all nonempty. We can assume that both $X$ and $Y$ properly contain $Z$ in $\langle X Y Z\rangle$. Necessarily $Z$ is disjoint from $(\langle X\rangle \cup\langle Y\rangle) \backslash\langle Z\rangle=(\langle X\rangle \cup\langle Y\rangle) \backslash\langle X Y Z\rangle$ by the 2-local cross-freeness of $\{X, Z\}$ and $\{Y, Z\}$. Hence $\{X, Z\}$ (or $\{Y, Z\}$ ) is cross-free on $S$; then we use (1).

(4). We can assume $X \subseteq^{*} Y$ by the 2-local cross-freeness of $\{X, Y\}$. Then we can also assume $X \subseteq^{*} Z$ or $Z \subseteq^{*} X$. If $X \subseteq^{*} Z$, then $X$ does not meet $(\langle Y\rangle \cup\langle Z\rangle) \backslash\langle X\rangle$, and $\{X, Y\}$ is cross-free on 
$S$; then we use (1). Hence suppose $Z \subseteq^{*} X$. By $X \subseteq^{*} Y$ and the 2-local cross-freeness of $\left\{X, Y_{i}\right\}$ for $i \in[k]$, it must hold that $X \subseteq^{*} Y_{i}$ for $i \in[k]$. Since $\left\{X, Y_{k}, Z\right\}$ is cross-free on $\langle X\rangle \cup\left\langle Y_{k}\right\rangle \cup\langle Z\rangle$, it holds that $Z \subseteq X \subseteq Y_{k}$ on $\langle X\rangle \cup\left\langle Y_{k}\right\rangle \cup\langle Z\rangle$. Here $\langle Z\rangle$ cannot meet $\left\langle Y_{i}\right\rangle \backslash\langle X\rangle$, since otherwise sequence $X Y, X Y_{1}, \ldots, X Y_{i}, X Z$ also forms a path in $G(\mathcal{G})$ and hence it holds that $X \subseteq^{*} Z$, a contradiction to $Z \subseteq^{*} X$. By this fact together with $\left\langle Y_{i} Y_{i+1}\right\rangle \backslash\langle X\rangle \neq \emptyset$, we can say $\left\langle Y_{i} Y_{i+1}\right\rangle \backslash\langle Z\rangle \neq \emptyset$. Hence, by $\langle X Y\rangle=\langle Z Y\rangle$, the sequence $Z Y, Z Y_{1}, \ldots, Z Y_{k}$ also forms a path in $G(\mathcal{G})$. By $Z \subseteq^{*} Y_{k}$ and the 2-local cross-freeness of $\left\{Z, Y_{i}\right\}$ for $i \in[k]$, we have $Z \subseteq^{*} Y$. Now $Z \subseteq^{*} X$ and $Z \subseteq^{*} Y$ hold. This means that $Z$ does not meet $(\langle X\rangle \cup\langle Y\rangle) \backslash\langle Z\rangle$, which implies that $\{Y, Z\}$ is cross-free on $S$; then we use (1).

We are now ready to give the proof of Proposition 4.3.

Proof of Proposition 4.3. We only prove that if $X \subseteq^{*} Y$, then $\mathcal{G}^{\prime}:=\mathcal{G} \backslash\{X, Y\} \cup\{X \cap Y, X \cup Y\}$ is 3-locally cross-free with $\mathcal{G}^{\prime} \sim \mathcal{G}$; the other case is similar.

First we prove $\mathcal{G}^{\prime} \sim \mathcal{G}$, that is, we show $X \sim X \cap Y$ and $Y \sim X \cup Y$. By $X \subseteq^{*} Y$, we have $X=X \cap Y$ on $\langle X\rangle \cup\langle Y\rangle$ and $Y=X \cup Y$ on $\langle X\rangle \cup\langle Y\rangle$. Furthermore, for any $p \in[r]$ with $A_{p} \cap(\langle X\rangle \cup\langle Y\rangle)=\emptyset$, $X \cap Y \supseteq A_{p}$ or $(X \cap Y) \cap A_{p}=\emptyset$ holds and $X \cup Y \supseteq A_{p}$ or $(X \cup Y) \cap A_{p}=\emptyset$ holds. This means $X \sim X \cap Y$ and $Y \sim X \cup Y$; then $\langle X\rangle=\langle X \cap Y\rangle$ and $\langle Y\rangle=\langle X \cup Y\rangle$ follow.

Next we show that $\mathcal{G}^{\prime}$ is 2-locally cross-free. Since the partition lines of $X$ and $Y$ are the same as those of $X \cap Y$ and $X \cup Y,\{X \cap Y, X \cup Y\}$ is also cross-free on $\langle X\rangle \cup\langle Y\rangle$. Hence $\{X \cap Y, X \cup Y\}$ is 2-locally cross-free. In the following, we prove that $\{X \cap Y, X \cup Y, Z\}$ is 2-locally cross-free for each $Z \in \mathcal{G} \backslash\{X, Y\}$.

If $\{X, Y\}$ is cross-free on $\langle X\rangle \cup\langle Y\rangle \cup\langle Z\rangle$, then the partition lines of $X$ and $Y$ on $\langle X\rangle \cup\langle Y\rangle \cup\langle Z\rangle$ are the same as those of $X \cap Y$ and $X \cup Y$. Hence, by the 2-local cross-freeness of $\mathcal{G}$, we obtain that $\{X \cap Y, X \cup Y, Z\}$ is also 2-locally cross-free. Therefore, it suffices to deal with the cases of (i) $\langle X Z\rangle=\langle Y Z\rangle=\emptyset$, (ii) $\langle X Z\rangle \neq \emptyset$ and $\langle X Y\rangle=\langle Y Z\rangle=\emptyset$, (iii) $\langle Y Z\rangle \neq \emptyset$ and $\langle X Y\rangle=\langle X Z\rangle=\emptyset$, and (iv) $\langle X Y\rangle=\langle Y Z\rangle=\langle Z X\rangle \neq \emptyset$. Indeed, for other cases, $\{X, Y, Z\}$ is cross-free on $\langle X\rangle \cup\langle Y\rangle \cup\langle Z\rangle$ by Lemma 4.6 (2), reducing to the cross-free case above.

(i). By the 2-local cross-freeness of $\mathcal{G}$, we have both $[X \supseteq\langle Z\rangle$ or $X \cap\langle Z\rangle=\emptyset]$ and $[Y \supseteq\langle Z\rangle$ or $Y \cap\langle Z\rangle=\emptyset]$. Hence both $[(X \cap Y) \supseteq\langle Z\rangle$ or $(X \cap Y) \cap\langle Z\rangle=\emptyset]$ and $[(X \cup Y) \supseteq\langle Z\rangle$ or $(X \cup Y) \cap\langle Z\rangle=\emptyset]$ hold. Therefore $\{X \cap Y, X \cup Y, Z\}$ is 2-locally cross-free.

(ii) and (iii). By symmetry, we show (ii) only. By $X \subseteq^{*} Y$, we have $Y \supseteq\langle X\rangle \backslash\langle Y\rangle$. By $\langle X Z\rangle \neq \emptyset$ and $\langle X Y\rangle=\langle Y Z\rangle=\emptyset$, it holds that $Y \cap(\langle Z\rangle \backslash\langle Y\rangle) \neq \emptyset$. By the 2-local cross-freeness of $\{Y, Z\}, Y$ must contain $\langle Z\rangle \backslash\langle Y\rangle$. Therefore $X \subseteq Y$ holds on $\langle X\rangle \cup\langle Y\rangle \cup\langle Z\rangle$, reducing to the cross-free case.

(iv). $\langle X Y\rangle=\langle Y Z\rangle=\langle Z X\rangle \neq \emptyset$ implies $\langle X Y Z\rangle \neq \emptyset$. Hence, by the 3-local cross-freeness of $\mathcal{G}$, $\{X, Y, Z\}$ is cross-free on $\langle X\rangle \cup\langle Y\rangle \cup\langle Z\rangle$, reducing to the cross-free case.

Finally, we show that $\mathcal{G}^{\prime}$ is 3-locally cross-free. Take distinct $S, T, U \in \mathcal{G}^{\prime}$ with $\langle S T U\rangle \neq \emptyset$. If $\{S, T, U\} \cap\{X \cap Y, X \cup Y\}=\emptyset$, then $\{S, T, U\}$ does not change in the construction of $\mathcal{G}^{\prime}$. Hence $\{S, T, U\}$ is cross-free on $\langle S\rangle \cup\langle T\rangle \cup\langle U\rangle$. If $|\{S, T, U\} \cap\{X \cap Y, X \cup Y\}|=1$, then $\{S, T, U\} \backslash\{X \cap$ $Y, X \cup Y\}$ is cross-free on $\langle S\rangle \cup\langle T\rangle \cup\langle U\rangle$. By the 2-local cross-freeness of $\mathcal{G}^{\prime}$ shown above and Lemma 4.6 (1), $\{S, T, U\}$ is also cross-free on $\langle S\rangle \cup\langle T\rangle \cup\langle U\rangle$. If $|\{S, T, U\} \cap\{X \cap Y, X \cup Y\}|=2$ (assume $S=X \cap Y$ and $T=X \cup Y$ ), then the partition lines of $X \cap Y$ and $X \cup Y$ on $\langle X\rangle \cup\langle Y\rangle \cup\langle U\rangle$ do not change in the construction of $\mathcal{G}^{\prime}$, since $\{X, Y, U\}$ is cross-free on $\langle X\rangle \cup\langle Y\rangle \cup\langle U\rangle$. Thus $\{X \cap Y, X \cup Y, U\}$ is cross-free on $\langle X\rangle \cup\langle Y\rangle \cup\langle U\rangle=\langle X \cap Y\rangle \cup\langle X \cup Y\rangle \cup\langle U\rangle$. This completes the proof of Proposition 4.3.

\subsection{Constructing 3-locally cross-free family}

Our final task is to show that, for an input $\mathcal{F}$ that is $\mathcal{A}$-equivalent to a 2-locally cross-free family, we can always construct a 3-locally cross-free family in polynomial time. Specifically, we use the 
LC-graph $G(\mathcal{F})$ introduced in Section 4.2, and construct an LC-labeling $s$ with the property that the family $\mathcal{F}^{s}$ in (4.4) transformed from $\mathcal{F}$ by $s$ is 3-locally cross-free. While the existence of an LC-labeling is guaranteed by the assumed $\mathcal{A}$-equivalence of $\mathcal{F}$ to a 2-locally cross-free family (Proposition 4.1), we need to exploit a certain intriguing structure inherent in an LC-graph before we can construct such a special LC-labeling.

Lemma 4.6 indicates that, more often than not, a triple $X, Y, Z$ in any 2-locally cross-free family is cross-free on $\langle X\rangle \cup\langle Y\rangle \cup\langle Z\rangle$. To construct a 3-locally cross-free family, particular cares are needed for those triples $X, Y, Z$ with $\langle X Y\rangle=\langle Y Z\rangle=\langle Z X\rangle \neq \emptyset$ for which there exists no path $\left(X Y, X Y_{1}, \ldots, X Y_{k}\right)$ satisfying $\langle X Y\rangle \neq\left\langle X Y_{k}\right\rangle \neq \emptyset$. Indeed, suppose that $\langle X Y\rangle,\langle Y Z\rangle$, and $\langle X Z\rangle$ are nonempty. If $\langle X Y\rangle \neq\langle Y Z\rangle$, then it holds that $\langle X Y\rangle \nsubseteq\langle Z\rangle$ or $\langle Y Z\rangle \nsubseteq\langle X\rangle$. Hence, by Lemma 4.6 (2), $\{X, Y, Z\}$ is cross-free on $\langle X\rangle \cup\langle Y\rangle \cup\langle Z\rangle$. If $\langle X Y\rangle=\langle Y Z\rangle=\langle Z X\rangle \neq \emptyset$ and there is a path $\left(X Y, X Y_{1}, \ldots, X Y_{k}\right)$ satisfying $\langle X Y\rangle \neq\left\langle X Y_{k}\right\rangle \neq \emptyset$, then, by the above argument for $\left\langle X Y_{k}\right\rangle \neq\langle Y Z\rangle$, $\left\{X, Y_{k}, Z\right\}$ is cross-free on $\langle X\rangle \cup\left\langle Y_{k}\right\rangle \cup\langle Z\rangle$. Hence, by Lemma 4.6 (4), $\{X, Y, Z\}$ is cross-free on $\langle X\rangle \cup\langle Y\rangle \cup\langle Z\rangle$.

This motivates the notion of special nodes and special connected components in the LC-graph $G(\mathcal{F})$. For distinct $X, Y \in \mathcal{F}$, define

$$
\begin{aligned}
R(X Y) & :=\left\{Z \in \mathcal{F} \mid \text { There is a path }\left(X Y, X Y_{1}, \ldots, X Z\right) \text { using only prefixed edges }\right\}, \\
R^{*}(X Y) & :=\{Z \in R(X Y) \mid\langle X Z\rangle \neq \emptyset\} .
\end{aligned}
$$

We say that a node $X Y$ (or an ordered pair of $X$ and $Y$ ) with $\langle X Y\rangle \neq \emptyset$ is special if $\langle X Z\rangle=\langle X Y\rangle$ holds for all $Z \in R^{*}(X Y)$. For $X, Y \in \mathcal{F}$ with $X Y$ and $Y X$ both being special, let $v(X Y)$ denote the connected component (as a set of nodes) containing $X Y$ (and $Y X)$ in $G(\mathcal{F})$. We call such a component special. Let $v^{*}(X Y)$ denote the set of nodes $Z W$ in $v(X Y)$ with $\langle Z W\rangle \neq \emptyset$.

A special component has an intriguing structure; the proof is given at the end of this section.

Proposition 4.7. If both $X Y$ and $Y X$ are special, then the following hold.

(1) $v(X Y)=\left(R^{*}(X Y) \times R(Y X)\right) \cup\left(R^{*}(Y X) \times R(X Y)\right)$.

(2) $v^{*}(X Y)=\left(R^{*}(X Y) \times R^{*}(Y X)\right) \cup\left(R^{*}(Y X) \times R^{*}(X Y)\right)$.

(3) If $Z W \in v^{*}(X Y)$, then $Z W$ is special and $\langle Z W\rangle=\langle X Y\rangle$.

For a special component $v=v(X Y)$, we call $\langle X Y\rangle$ the center of $v$; this is well-defined by Proposition 4.7 (3). For $Q \subseteq[r]$, the set $C$ of all special components whose center coincides with $A_{Q}$ is called the $Q$-flower if the size $|C|$ is at least two. The following proposition gives a concrete representation of the $Q$-flower; the proof is given at the end of this section.

Proposition 4.8. The $Q$-flower is given as

$$
\left\{v\left(X_{i} X_{j}\right) \mid 1 \leq i<j \leq p\right\}
$$

for some $p \geq 3$ and distinct $X_{1}, X_{2}, \ldots, X_{p} \in \mathcal{F}$ such that $R\left(X_{i} X_{j}\right)=R\left(X_{i^{\prime}} X_{j}\right)$ for all $i$ and $i^{\prime}<j$, and $R\left(X_{i} X_{j}\right) \cap R\left(X_{i^{\prime}} X_{j^{\prime}}\right)=\emptyset$ for all distinct $j, j^{\prime} \in[p], i<j$, and $i^{\prime}<j^{\prime}$.

The above $X_{1}, X_{2}, \ldots, X_{p}$ are called the representatives of the $Q$-flower.

Example 4.9. We give a new $\mathcal{A}$-cut family $\mathcal{F}$ appearing $Q$-flowers in the LC-graph $G(\mathcal{F})$. Let $\mathcal{F}=\{S, T, U, V, X, Y, Z\}$ be the $\mathcal{A}$-cut family illustrated in Figure 6, its LC-graph $G(\mathcal{F})$ being illustrated in Figure 7. In $G(\mathcal{F})$, there are six special components $v(S T)(=v(S V)), v(S X), v(T X)(=$ $v(V X)), v(X Y), v(X Z)$, and $v(Y Z)$. We can see that $\{v(S T), v(S X), v(T X)\}$ is the $\{1\}$-flower and $\{v(X Y), v(X Z), v(Y Z)\}$ is the $\{2\}$-flower.

A component $v$ is said to be fixed if $v$ contains a fixed node, and free otherwise. A special component $v(X Y)$ in the $Q$-flower is free if and only if the partition lines of $X^{\prime}$ and $Y^{\prime}$ on $A_{Q}$ are 


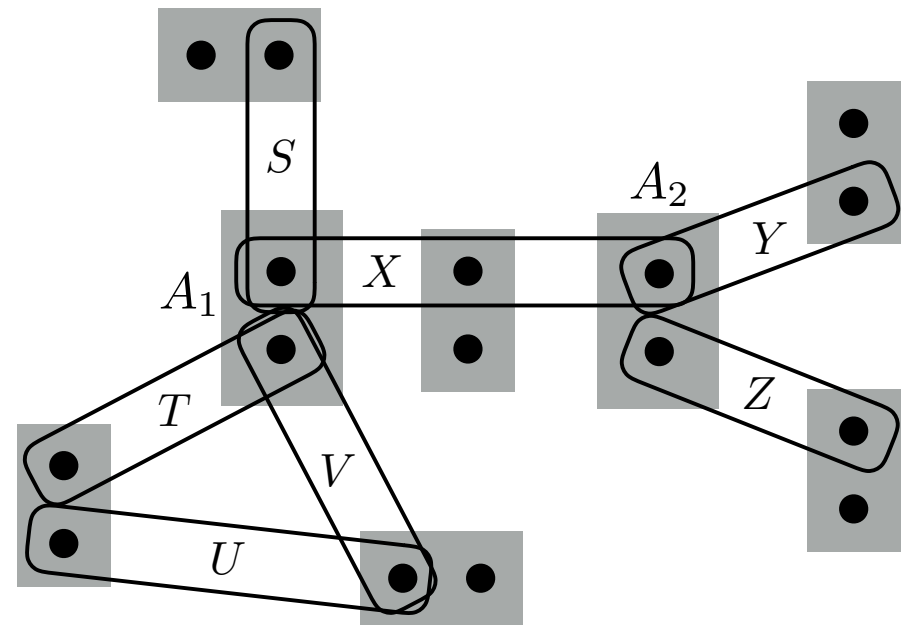

Fig. 6. Black nodes indicate elements of $[n]$, gray rectangles indicate elements of $\mathcal{A}$, and solid curves indicate elements of $\mathcal{F}=\{S, T, U, V, X, Y, Z\}$. It holds that $A_{1}=\langle S T\rangle=\langle T X\rangle=\langle S X\rangle$ and $A_{2}=\langle X Y\rangle=\langle Y Z\rangle=$ $\langle X Z\rangle$.
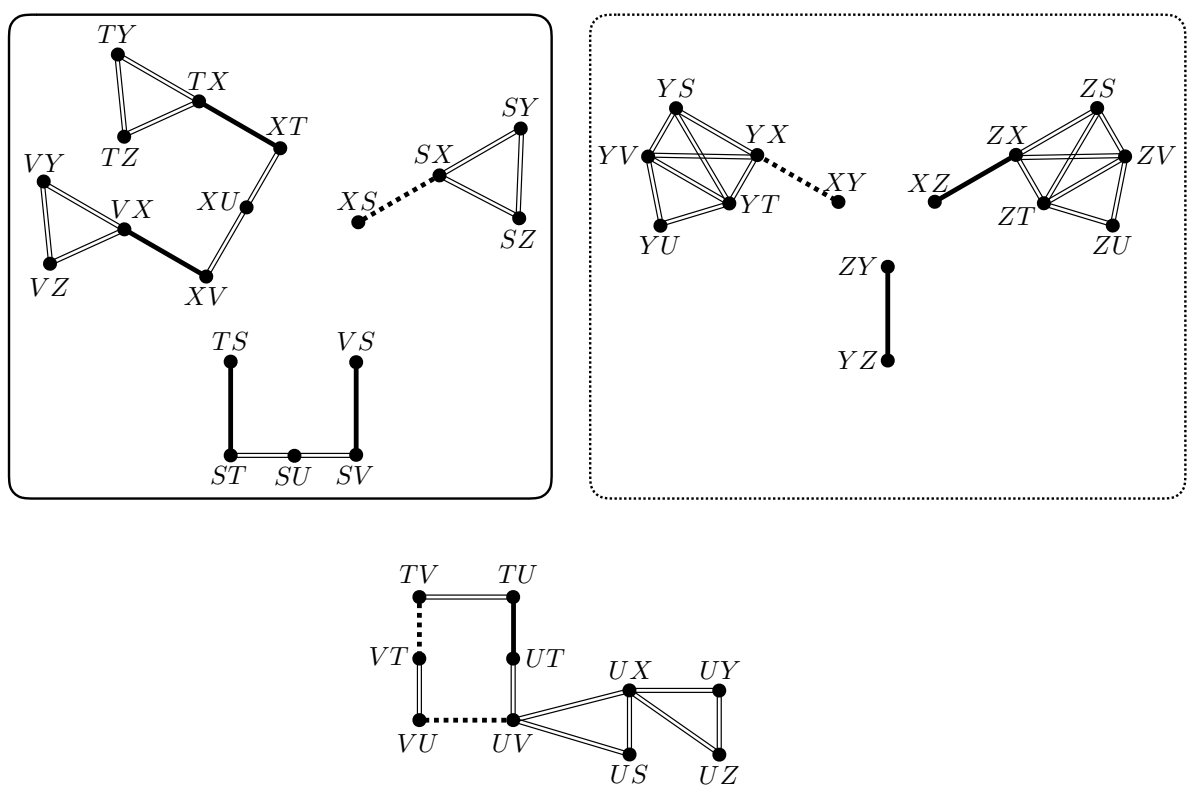

Fig. 7. The LC-graph $G(\mathcal{F})$ for $\mathcal{F}=\{S, T, U, V, X, Y, Z\}$ defined in Figure 6. $\{1\}$-flower (resp. $\{2\}$-flower) consists of the connected components included in the left solid curve (resp. the right dotted curve).

the same for all $X^{\prime} \in R^{*}(Y X)$ and $Y^{\prime} \in R^{*}(X Y)$. A free $Q$-flower is a maximal set of free components in the $Q$-flower such that the partition lines on $A_{Q}$ are the same. Now the set of free components of the $Q$-flower is partitioned to free $Q$-flowers each of which is represented as

$$
\left\{v\left(X_{i_{s}} X_{i_{t}}\right) \mid 1 \leq s<t \leq q\right\}
$$


with a subset $\left\{X_{i_{1}} X_{i_{2}}, \ldots, X_{i_{q}}\right\}$ of the representatives. A free $Q$-flower (for some $Q \subseteq[r]$ ) is also called a free flower.

We now provide a polynomial-time algorithm to construct a 3-locally cross-free family $\mathcal{F}^{s}$ by defining an appropriate LC-labeling $s$.

\section{Algorithm 5 (for constructing a 3-locally cross-free family):}

Step 0: Determine whether there exists a 2-locally cross-free family $\mathcal{A}$-equivalent to $\mathcal{F}$. If not, then output " $\mathcal{F}$ is not laminarizable" and stop.

Step 1: For all fixed nodes $X Y$, define $s(X Y)$ according to (4.1). By a breath-first search, define $s$ on all other nodes in fixed components appropriately.

Step 2: For each component $v$ which is free and not special, take any node $X Y$ in $v$. Define $s(X Y)$ as 0 or 1 arbitrarily, and define $s(Z W)$ appropriately for all nodes $Z W$ in $v$. Then all the remaining (undefined) components are special and free.

Step 3: For each free flower, which is assumed to be represented as $\left\{v\left(X_{i} X_{j}\right) \mid 1 \leq i<j \leq q\right\}$, do the following:

3-1: Define the value of $s\left(X_{i} X_{j}\right)$ for $i, j \in[q]$ with $i<j$ so that $\left\{X_{1}^{s}, X_{2}^{s}, \ldots, X_{q}^{s}\right\}$ is cross-free on $\bigcup_{i \in[q]}\left\langle X_{i}\right\rangle$; such a labeling is given, for example, as

$$
s\left(X_{i} X_{j}\right):= \begin{cases}0 & \text { if } X_{i}=X_{1} \text { on } A_{Q}, \\ 1 & \text { if } X_{i}=\bar{X}_{1} \text { on } A_{Q},\end{cases}
$$

where $A_{Q}$ is the center of the free flower.

3-2: Define $s(Z W)$ appropriately for all $Z W \in v\left(X_{i} X_{j}\right)$.

Step 4: Output $\mathcal{F}^{s}$.

Example 4.10. We consider $\mathcal{F}=\left\{X_{1}, X_{2}, \ldots, X_{7}\right\}$ in Figure 6 and its LC-graph $G(\mathcal{F})$ in Figure 7 . We execute Algorithm 5 for $G(\mathcal{F})$.

We can easily determine that there exists a 2-locally cross-free family $\mathcal{A}$-equivalent to $\mathcal{F}$, and that there is no fixed node in $G(\mathcal{F})$. In Step 2, there is one component $v$ which is free and not special in $G(\mathcal{F})$ (the one at the bottom in Figure 7). We take, say, $X_{5} X_{6} \in v$ and define $s\left(X_{5} X_{6}\right):=1$. Then, by (4.2) and (4.3), we have $s\left(X^{\prime} Y^{\prime}\right)=0$ for $X^{\prime} Y^{\prime} \in\left\{X_{6} X_{5}, X_{6} X_{7}\right\}$ and $s\left(X^{\prime} Y^{\prime}\right)=1$ for other nodes in $v$ We consider Step 3. Two flowers ( $\{1\}$-flower and $\{2\}$-flower) exist in $G(\mathcal{F})$ (see Example 4.9). Hence, for the $\{1\}$-flower $\left\{v\left(X_{1} X_{2}\right), v\left(X_{1} X_{3}\right), v\left(X_{2} X_{3}\right)\right\}$, we define $s\left(X_{1} X_{2}\right)=s\left(X_{1} X_{3}\right)=s\left(X_{2} X_{3}\right):=0$, and for the $\{2\}$-flower $\left\{v\left(X_{1} X_{4}\right), v\left(X_{1} X_{5}\right), v\left(X_{4} X_{5}\right)\right\}$, we define $s\left(X_{1} X_{4}\right)=s\left(X_{1} X_{5}\right)=s\left(X_{4} X_{5}\right):=0$. Then we define the other values according to (4.2) and (4.3). Thus we can construct an LC-labeling inducing a 3-locally cross-free family.

Proposition 4.11. The output $\mathcal{F}^{s}$ is 3-locally cross-free, and Algorithm 5 runs in $O\left(n^{4}\right)$ time.

Proof. We show the 3-local cross-freeness of $\mathcal{F}^{s}$. Recall that $\mathcal{F}^{s}$ is 2-locally cross-free and $\left\langle X^{s}\right\rangle=\langle X\rangle$ for $X^{s} \in \mathcal{F}^{s}$ (and $X \in \mathcal{F}$ ). Take any triple $\left\{X^{s}, Y^{s}, Z^{s}\right\}$ with $\langle X Y Z\rangle \neq \emptyset$. It suffices to deal with the case of $\langle X Y\rangle=\langle Y Z\rangle=\langle Z X\rangle \neq \emptyset$ by Lemma 4.6 (2). If $X Y$ is not special, there is a path $\left(X Y, X Y_{1}, \ldots, X Y_{k}\right)$ in $G(\mathcal{F})$ such that $\emptyset \neq\left\langle X Y_{k}\right\rangle \neq\langle X Y\rangle=\langle X Z\rangle$. Here $\left\langle X Y_{k}\right\rangle \nsubseteq\langle Z\rangle$ or $\langle X Z\rangle \nsubseteq$ $\langle Y\rangle$ holds. By Lemma 4.6 (2), $\left\{X^{s}, Y_{k}^{s}, Z^{s}\right\}$ is cross-free on $\langle X\rangle \cup\left\langle Y_{k}\right\rangle \cup\langle Z\rangle$. Hence, by Lemma 4.6 (4), $\{X, Y, Z\}$ is cross-free on $\langle X\rangle \cup\langle Y\rangle \cup\langle Z\rangle$. Therefore, we assume that $X Y, Y X, Y Z, Z Y, Z X, X Z$ are special.

We can suppose that $X Y, Y Z, Z X$ belong to special components of the $Q$-flower $\left\{v\left(X_{i} X_{j}\right) \mid 1 \leq\right.$ $i<j \leq p\}$, i.e., $\langle X Y\rangle=\langle Y Z\rangle=\langle Z X\rangle=A_{Q}$. By Proposition 4.8, we can assume $X \in R^{*}\left(X_{k} X_{i}\right)$ $Y \in R^{*}\left(X_{i} X_{j}\right)$, and $Z \in R^{*}\left(X_{j} X_{k}\right)$ for distinct $i, j, k \in[q]$ with $i<j<k$.

Suppose that $v\left(X_{i} X_{j}\right), v\left(X_{i} X_{k}\right)$, or $v\left(X_{j} X_{k}\right)$ is fixed. Then we can assume that there is $\hat{X} \in$ $R^{*}\left(X_{k} X_{i}\right)$ such that the partition lines of $\hat{X}, Y, Z$ are not the same. By Lemma $4.6(3),\left\{\hat{X}^{s}, Y^{s}, Z^{s}\right\}$ is 
cross-free on $\langle\hat{X}\rangle \cup\langle Y\rangle \cup\langle Z\rangle$. Furthermore, since there is a path $\left(Y X=Y X_{0}, Y X_{1}, \ldots, Y X_{k}=Y \hat{X}\right)$, $\left\{X^{s}, Y^{s}, Z^{s}\right\}$ is cross-free on $\langle X\rangle \cup\langle Y\rangle \cup\langle Z\rangle$ by Lemma 4.6 (4).

Suppose that $v\left(X_{i} X_{j}\right), v\left(X_{i} X_{k}\right)$, and $v\left(X_{j} X_{k}\right)$ are free. Then $v\left(X_{i} X_{j}\right), v\left(X_{i} X_{k}\right), v\left(X_{j} X_{k}\right)$ are contained in the same free $Q$-flower. By the definition of $s$ (cf. (4.6)), $\left\{X_{i}^{s}, X_{j}^{s}, X_{k}^{s}\right\}$ is cross-free on $\left\langle X_{i}\right\rangle \cup\left\langle X_{j}\right\rangle \cup\left\langle X_{k}\right\rangle$. By applying repeatedly Lemma 4.6 (4), $\left\{X^{s}, Y^{s}, Z^{s}\right\}$ is cross-free on $\langle X\rangle \cup\langle Y\rangle \cup\langle Z\rangle$.

Finally we see the running-time of Algorithm 5. By the argument at the end of Section 4.2, Step 0 can be done in $O\left(n^{4}\right)$ time. We can also obtain an appropriate value of each $s(X Y)$ in Steps 1-3 in $O\left(n^{4}\right)$ time. From $s$, we can construct $\mathcal{F}^{s}$ in $O(|V(\mathcal{F})|)=O\left(n^{2}\right)$ time. Thus the running-time of Algorithm 5 is bounded by $O\left(n^{4}\right)$.

By Propositions 4.4 and 4.11, we obtain the following theorem.

Theorem 4.12. Algorithms 4 and 5 solve Laminarization in $O\left(n^{4}\right)$ time.

The rest of this section is devoted to proving Propositions 4.7 and 4.8. First we show a key lemma about special nodes.

LEMмA 4.13. If $X Y$ is special and $\langle X Y\rangle=\left\langle X^{\prime} Y\right\rangle$ for some $X^{\prime}$, then $R\left(X^{\prime} Y\right) \subseteq R(X Y)$, and $\left\langle X^{\prime} Z\right\rangle \supseteq\langle X Z\rangle$ for any $Z \in R\left(X^{\prime} Y\right)$.

Proof. We prove $Y_{k} \in R(X Y)$ and $\left\langle X^{\prime} Y_{k}\right\rangle \supseteq\left\langle X Y_{k}\right\rangle$ by induction on the length $k$ of a path $\left(X^{\prime} Y=X^{\prime} Y_{0}, X^{\prime} Y_{1}, \ldots, X^{\prime} Y_{k}\right)$. For $k=0$, we have $Y_{0}=Y \in R(X Y)$ and $\left\langle X^{\prime} Y_{0}\right\rangle \supseteq\left\langle X Y_{0}\right\rangle$. For the induction step, suppose that $Y_{k} \in R(X Y)$ and $\left\langle X^{\prime} Y_{k}\right\rangle \supseteq\left\langle X Y_{k}\right\rangle$ for $k \geq 0$. Since a prefixed edge $\left\{X^{\prime} Y_{k}, X^{\prime} Y_{k+1}\right\}$ exists, we have $\left\langle Y_{k} Y_{k+1}\right\rangle \backslash\left\langle X^{\prime} Y_{k}\right\rangle \neq \emptyset$. Then $Y_{k+1} \neq X$ holds. Indeed, if $Y_{k+1}=X$, then $\left\langle X Y_{k}\right\rangle \backslash\left\langle X^{\prime} Y_{k}\right\rangle \neq \emptyset$, a contradiction to $\left\langle X^{\prime} Y_{k}\right\rangle \supseteq\left\langle X Y_{k}\right\rangle$. By $\left\langle X^{\prime} Y_{k}\right\rangle \supseteq\left\langle X Y_{k}\right\rangle$, we obtain $\left\langle Y_{k} Y_{k+1}\right\rangle \backslash\left\langle X Y_{k}\right\rangle \neq \emptyset$. Hence there is a prefixed edge $\left\{X Y_{k}, X Y_{k+1}\right\}$. This means $Y_{k+1} \in R(X Y)$.

Suppose, to the contrary, that $\left\langle X^{\prime} Y_{k+1}\right\rangle \nsupseteq\left\langle X Y_{k+1}\right\rangle$, i.e., $\left\langle X Y_{k+1}\right\rangle \backslash\left\langle X^{\prime} Y_{k+1}\right\rangle \neq \emptyset$ holds. Note that $\left\langle X Y_{k+1}\right\rangle \backslash\left\langle X^{\prime} Y_{k+1}\right\rangle=\left\langle X Y_{k+1}\right\rangle \backslash\left\langle X X^{\prime}\right\rangle$ holds. Furthermore, by $\langle X Y\rangle=\left\langle X^{\prime} Y\right\rangle$, we obtain $\left\langle X X^{\prime}\right\rangle \supseteq\langle X Y\rangle$. Hence we have $\left\langle X Y_{k+1}\right\rangle \backslash\langle X Y\rangle \neq \emptyset$. However, since $X Y$ is special and $Y_{k+1} \in R(X Y)$, it must hold that $\left\langle X Y_{k+1}\right\rangle=\langle X Y\rangle$ or $\left\langle X Y_{k+1}\right\rangle=\emptyset$; this is a contradiction.

Proof of Proposition 4.7. First we show the following three claims.

Claim 1. $R(X Y) \cap R(Y X)=\emptyset$.

Proof. Suppose, to the contrary, that $R(X Y) \cap R(Y X) \neq \emptyset$. For each $Z \in R(X Y)$, we have $\langle X Z\rangle \subseteq\langle Y Z\rangle$ since $\langle X Z\rangle=\langle X Y\rangle$ or $\langle X Z\rangle=\emptyset$.

Let $Z \in R(X Y) \cap R(Y X)$ be an element such that the length $k$ of a path $(Y X, \ldots, Y Z)$ in $G(\mathcal{F})$ is shortest. If $k \geq 2$, there is a prefixed edge $\left\{Y Z_{k}, Y Z_{k-1}\right\}$ and $Z_{k-1} \neq X$. That is $\left\langle Z_{k} Z_{k-1}\right\rangle\left\langle\left\langle Y Z_{k}\right\rangle \neq \emptyset\right.$. By $\left\langle X Z_{k}\right\rangle \subseteq\left\langle Y Z_{k}\right\rangle$, we obtain $\left\langle Z_{k} Z_{k-1}\right\rangle \backslash\left\langle X Z_{k}\right\rangle \neq \emptyset$. Hence a prefixed edge $\left\{X Z_{k}, X Z_{k-1}\right\}$ exists. This means $Z_{k-1} \in R(X Y) \cap R(Y X)$, which contradicts the minimality of $Z=Z_{k}$. Therefore a prefixed edge $\{Y X, Y Z\}$ exists for some $Z \in R(X Y) \cap R(Y X)$. That is, $\langle X Z\rangle \backslash\langle X Y\rangle \neq \emptyset$. Hence we obtain $\emptyset \neq\langle X Z\rangle \neq\langle X Y\rangle$. This contradicts the assumption that $X Y$ is special.

Claim 2. For any $Y^{\prime} \in R^{*}(X Y)$, it holds that $R(Y X)=R\left(Y^{\prime} X\right)$, and $\left\langle Y^{\prime} Z\right\rangle=\langle Y Z\rangle$ for any $Z \in R(Y X)=R\left(Y^{\prime} X\right)$.

Proof. If $R^{*}(X Y)=\{Y\}$, the proof is trivial. Suppose $R^{*}(X Y) \backslash\{Y\} \neq \emptyset$. Take any $Y^{\prime} \in R^{*}(X Y) \backslash$ $\{Y\}$. Then there is a swapped edge $\left\{X Y^{\prime}, Y^{\prime} X\right\}$. Therefore, for all $Z \in R\left(Y^{\prime} X\right), X Y$ and $Y^{\prime} Z$ are connected. Since $X Y$ is special, it holds that $\langle Y X\rangle=\left\langle Y^{\prime} X\right\rangle$. Since $Y X$ is special and $\langle Y X\rangle=\left\langle Y^{\prime} X\right\rangle$, by Lemma 4.13, we have $R\left(Y^{\prime} X\right) \subseteq R(Y X)$ and $\left\langle Y^{\prime} Z\right\rangle \supseteq\langle Y Z\rangle$ for all $Z \in R\left(Y^{\prime} X\right)$.

In the following, we prove that, for each $Z \in R(Y X)$, it holds that $Z \in R\left(Y^{\prime} X\right)$ and $\left\langle Y^{\prime} Z\right\rangle \subseteq\langle Y Z\rangle$, which imply $R\left(Y^{\prime} X\right)=R(Y X)$ and $\left\langle Y^{\prime} Z\right\rangle=\langle Y Z\rangle$. We show this by induction on the length of a 
path $\left(Y X=Y X_{0}, Y X_{1}, \ldots, Y X_{k+1}=Y Z\right)$. For $X_{0}$, we have $R\left(Y^{\prime} X\right) \ni X=X_{0}$ and $\left\langle Y X_{0}\right\rangle=\left\langle Y^{\prime} X_{0}\right\rangle$. Suppose $R\left(Y^{\prime} X\right) \ni X_{k}$ and $\left\langle Y X_{k}\right\rangle \supseteq\left\langle Y^{\prime} X_{k}\right\rangle$ by induction. Since a prefixed edge $\left\{Y X_{k}, Y X_{k+1}\right\}$ exists, we have $\left\langle X_{k} X_{k+1}\right\rangle \backslash\left\langle Y X_{k}\right\rangle \neq \emptyset$. By $\left\langle Y X_{k}\right\rangle \supseteq\left\langle Y^{\prime} X_{k}\right\rangle$, we obtain $\left\langle X_{k} X_{k+1}\right\rangle \backslash\left\langle Y^{\prime} X_{k}\right\rangle \neq \emptyset$. Hence there is a prefixed edge $\left\{Y^{\prime} X_{k}, Y^{\prime} X_{k+1}\right\}$. This means $R\left(Y^{\prime} X\right) \ni X_{k+1}=Z$.

Suppose, to the contrary, that $\left\langle Y X_{k+1}\right\rangle \nsupseteq\left\langle Y^{\prime} X_{k+1}\right\rangle$, i.e., $\left\langle Y^{\prime} X_{k+1}\right\rangle \backslash\left\langle Y X_{k+1}\right\rangle \neq \emptyset$ holds. Then there is a prefixed edge $\left\{Y X_{k+1}, Y Y^{\prime}\right\}$. Hence we have $R(Y X) \ni Y^{\prime}$. However this contradicts $R(Y X) \not \nexists Y^{\prime}$ by Claim 1 and $R(X Y) \ni Y^{\prime}$. Therefore we obtain $\left\langle Y X_{k+1}\right\rangle \supseteq\left\langle Y^{\prime} X_{k+1}\right\rangle$.

Claim 3. For $Z W \in v(X Y)$, there is a path from $X Y$ or $Y X$ to $Z W$ containing at most one swapped edge.

Proof. Suppose, to the contrary, that, for some $Z W$, all paths from $X Y$ to $Z W$ and from $Y X$ to $Z W$ use at least two swapped edges. Take such a path $P$ with a minimum number of swapped edges. Denote the number of swapped edges in $P$ by $k(\geq 2)$. Without loss of generality, we assume that $P$ is a path from $X Y$ to $Z W$. By $k \geq 2, P$ has a subpath $\left(X Y=X_{0} Y_{0}, \ldots, X_{0} Y_{1}, Y_{1} X_{0}, \ldots, Y_{1} X_{1}, X_{1} Y_{1}\right)$ Note that $Y_{1} \in R^{*}(X Y)$, and $X_{1} \in R^{*}\left(Y_{1} X\right)=R^{*}(Y X)$ by Claim 2. Hence there is a path from $Y X$ to $X_{1} Y_{1}$ using only one swapped edge. Indeed, $\left(Y X=Y_{0} X_{0}, \ldots, Y_{0} X_{1}, X_{1} Y_{0}, \ldots, X_{1} Y_{1}\right)$ is such a path. This means that there is a path from $Y X$ to $Z W$ with $k-1$ swapped edges, a contradiction to the minimality of $P$.

We are now ready to show the statement of Proposition 4.7 (1). If $Z \in R^{*}(X Y)$ and $W \in R(Y X)$, then there is a path in $G(\mathcal{F})$ such as $(X Y, \ldots, X Z, Z X, \ldots, Z W)$ since $R(Y X)=R(Z X)$ by Claim 2, implying $Z W \in v(X Y)$. Conversely, if $Z W \in v(X Y)$, then there is a path from $X Y$ or $Y X$ to $Z W$ with at most one swapped edge by Claim 3. We may assume that there is such a path $P$ from $X Y$ to $Z W$. If $P$ has no swapped edge, then $Z=X \in R^{*}(Y X)$ and $W \in R(X Y)$ hold. If $P$ has exactly one swapped edge, then $Z \in R^{*}(X Y)$ and $W \in R(Z X)=R(Y X)$ by Claim 2. Thus we obtain Proposition 4.7 (1).

Next we show Proposition 4.7 (3). If $Z W \in v(X Y)$, then there is a path from $X Y$ or $Y X$ to $Z W$ with at most one swapped edge by Claim 3. We may assume that there is such a path $P$ from $X Y$ to $Z W$. If $P$ has no swapped edge, then $\langle Z W\rangle=\langle X Y\rangle$ or $\langle Z W\rangle=\emptyset$ holds since $X Y$ is special. If $P$ has one swapped edge, then $\langle Z W\rangle=\langle Y W\rangle$ holds by Claim 2 and $\langle Y W\rangle=\langle X Y\rangle$ or $\langle Y W\rangle=\emptyset$ holds since $Y X$ is special. Therefore, if $Z W \in v^{*}(X Y)$, then $\langle Z W\rangle=\langle X Y\rangle$, and $Z W$ is obviously special. Thus we obtain Proposition 4.7 (3).

Finally we show Proposition 4.7 (2). For every $Z \in R^{*}(X Y)$ and $W \in R^{*}(Y X)$, we have $\langle Z\rangle \supseteq$ $\langle X Y\rangle \subseteq\langle W\rangle$ by (3). Hence $\langle Z W\rangle \neq \emptyset$, implying $Z W \in v^{*}(X Y)$. Conversely, let $Z W \in v^{*}(X Y)$. By Proposition 4.7 (1), we may assume $Z \in R^{*}(X Y)$ and $W \in R(Y X)$. Since $\langle Z W\rangle \neq \emptyset,\langle Z W\rangle=\langle X Y\rangle$ holds by Proposition 4.7 (3). Hence $\langle W\rangle \cap\langle Y\rangle \supseteq\langle X Y\rangle \neq \emptyset$. This means $W \in R^{*}(Y X)$.

Proof of Proposition 4.8. Let $v\left(X_{1} X_{2}\right)$ be a special connected component with $\left\langle v\left(X_{1} X_{2}\right)\right\rangle=$ $A_{Q}$. Take any special connected component $v\left(Y_{1} Y_{2}\right)$ with $\left\langle v\left(Y_{1} Y_{2}\right)\right\rangle=A_{Q}$. It suffices to show that, (i) if $R\left(X_{1} X_{2}\right) \cap R\left(Y_{1} Y_{2}\right) \neq \emptyset$, we have $R\left(X_{1} X_{2}\right)=R\left(Y_{1} Y_{2}\right)$ (this implies $v\left(Y_{1} Y_{2}\right)=v\left(Y_{1} X_{2}\right)$ by $\left.Y_{2} \in R^{*}\left(X_{1} X_{2}\right)\right)$, and (ii) if $R\left(X_{1} X_{2}\right) \cap R\left(Y_{1} Y_{2}\right)=\emptyset$, there exists a special connected component $v\left(X_{2} Y_{2}\right)$ with $\left\langle v\left(X_{2} Y_{2}\right)\right\rangle=A_{Q}, R\left(X_{2} Y_{2}\right)=R\left(Y_{1} Y_{2}\right)$, and $R\left(Y_{2} X_{2}\right)=R\left(X_{1} X_{2}\right)$.

(i). If there exists $Z \in R^{*}\left(X_{1} X_{2}\right) \cap R^{*}\left(Y_{1} Y_{2}\right)$, then $X_{1} Z$ and $Y_{1} Z$ are special and $\left\langle X_{1} Z\right\rangle=\left\langle Y_{1} Z\right\rangle$ (= $\left.A_{Q}\right)$. Hence, by Lemma 4.13, we have $R\left(X_{1} Z\right) \subseteq R\left(Y_{1} Z\right)$ and $R\left(X_{1} Z\right) \supseteq R\left(Y_{1} Z\right)$, i.e., $R\left(X_{1} Z\right)=R\left(Y_{1} Z\right)$. This implies $R\left(X_{1} X_{2}\right)=R\left(X_{1} Z\right)=R\left(Y_{1} Z\right)=R\left(Y_{1} Y_{2}\right)$, as required. Thus, in the following, we show that there exists $Z \in R^{*}\left(X_{1} X_{2}\right) \cap R^{*}\left(Y_{1} Y_{2}\right)$.

Suppose, to the contrary, $R^{*}\left(X_{1} X_{2}\right) \cap R^{*}\left(Y_{1} Y_{2}\right)=\emptyset$. Note that $R^{*}\left(X_{1} X_{2}\right) \cap R^{*}\left(Y_{1} Y_{2}\right)=\emptyset$ implies $R^{*}\left(X_{1} X_{2}\right) \cap R\left(Y_{1} Y_{2}\right)=R\left(X_{1} X_{2}\right) \cap R^{*}\left(Y_{1} Y_{2}\right)=\emptyset$. Indeed, each $Z \in R^{*}\left(X_{1} X_{2}\right) \cap R\left(Y_{1} Y_{2}\right)$ satisfies $Z \supseteq A_{Q}$ by $Z \in R^{*}\left(X_{1} X_{2}\right)$. Hence $Z \in R^{*}\left(Y_{1} Y_{2}\right)$ holds by $Z \in R\left(Y_{1} Y_{2}\right)$ and $\left\langle Y_{1} Z\right\rangle \neq \emptyset$. Let $Z \in R\left(X_{1} X_{2}\right) \cap R\left(Y_{1} Y_{2}\right)=\left(R\left(X_{1} X_{2}\right) \cap R\left(Y_{1} Y_{2}\right)\right) \backslash\left(R^{*}\left(X_{1} X_{2}\right) \cup R^{*}\left(Y_{1} Y_{2}\right)\right)$ be an element such that the 
length of a path $\left(X_{1} X_{2}=X_{1} Z_{0}, X_{1} Z_{1}, \ldots, X_{1} Z_{k}=X_{1} Z\right)$ is shortest; by the assumption, $k \geq 1$. Since a prefixed edge $\left\{X_{1} Z_{k}, X_{1} Z_{k-1}\right\}$ exists, we have $\left\langle Z_{k} Z_{k-1}\right\rangle \backslash\left\langle X_{1} Z_{k}\right\rangle \neq \emptyset$. Furthermore, by $\left\langle X_{1} Z_{k}\right\rangle=$ $\left\langle Y_{1} Z_{k}\right\rangle=\emptyset$, we obtain $\left\langle Z_{k} Z_{k-1}\right\rangle \backslash\left\langle Y_{1} Z_{k}\right\rangle \neq \emptyset$. This means that a prefixed edge $\left\{Y_{1} Z_{k}, Y_{1} Z_{k-1}\right\}$ exists and $Z_{k-1} \in R\left(X_{1} X_{2}\right) \cap R\left(Y_{1} Y_{2}\right)$ holds, a contradiction to the minimality of $k$.

(ii). First we show that $\left\langle X_{2}^{\prime} Y_{2}^{\prime}\right\rangle=A_{Q}$ or $\left\langle X_{2}^{\prime} Y_{2}^{\prime}\right\rangle=\emptyset$ holds for any $X_{2}^{\prime} \in R\left(X_{1} X_{2}\right)$ and $Y_{2}^{\prime} \in R\left(Y_{1} Y_{2}\right)$. Since, for any $Z \in R\left(X_{1} X_{2}\right) \cup R\left(Y_{1} Y_{2}\right),\langle Z\rangle \supseteq A_{Q}$ or $\langle Z\rangle \cap A_{Q}=\emptyset$ holds by Proposition 4.7 (3), we have $\left\langle X_{2}^{\prime} Y_{2}^{\prime}\right\rangle \supseteq A_{Q}$ or $\left\langle X_{2}^{\prime} Y_{2}^{\prime}\right\rangle \cap A_{Q}=\emptyset$ for each $X_{2}^{\prime} \in R\left(X_{1} X_{2}\right)$ and $Y_{2}^{\prime} \in R\left(Y_{1} Y_{2}\right)$ with $\left\langle X_{2}^{\prime} Y_{2}^{\prime}\right\rangle \neq \emptyset$. Suppose, to the contrary, that there exist $X_{2}^{\prime} \in R\left(X_{1} X_{2}\right)$ and $Y_{2}^{\prime} \in R\left(Y_{1} Y_{2}\right)$ with $\emptyset \neq\left\langle X_{2}^{\prime} Y_{2}^{\prime}\right\rangle \neq A_{Q}$. Then $\left\langle X_{2}^{\prime} Y_{2}^{\prime}\right\rangle \supsetneq A_{Q}$ or $\left\langle X_{2}^{\prime} Y_{2}^{\prime}\right\rangle \cap A_{Q}=\emptyset$ holds. Hence we have $\left\langle X_{2}^{\prime} Y_{2}^{\prime}\right\rangle \backslash\left\langle X_{1} X_{2}^{\prime}\right\rangle \neq \emptyset$ by $\left\langle X_{1} X_{2}^{\prime}\right\rangle=A_{Q}$ or $\left\langle X_{1} X_{2}^{\prime}\right\rangle=\emptyset$. This means that there is a prefixed edge $\left\{X_{1} X_{2}^{\prime}, X_{1} Y_{2}^{\prime}\right\}$ and $R\left(X_{1} X_{2}\right) \cap R\left(Y_{1} Y_{2}\right) \neq \emptyset$ holds, a contradiction.

By $\left\langle X_{2}\right\rangle \supseteq A_{Q} \subseteq\left\langle Y_{2}\right\rangle$, we have $\left\langle X_{2} Y_{2}\right\rangle \neq \emptyset$. Hence, by the above argument, we obtain $\left\langle X_{2} Y_{2}\right\rangle=$ $A_{Q}$. Furthermore $Y_{1} Y_{2}$ is special and $\left\langle Y_{1} Y_{2}\right\rangle=\left\langle X_{2} Y_{2}\right\rangle$ holds. By Lemma 4.13, we obtain $R\left(X_{2} Y_{2}\right) \subseteq$ $R\left(Y_{1} Y_{2}\right)$. By $\left\langle X_{2} Z\right\rangle=A_{Q}$ or $\left\langle X_{2} Z\right\rangle=\emptyset$ for every $Z \in R\left(X_{2} Y_{2}\right) \subseteq R\left(Y_{1} Y_{2}\right)$, it holds that $X_{2} Y_{2}$ is special. Furthermore, since $X_{2} Y_{2}$ is special, we also obtain $R\left(X_{2} Y_{2}\right) \supseteq R\left(Y_{1} Y_{2}\right)$ by Lemma 4.13. Hence $R\left(X_{2} Y_{2}\right)=R\left(Y_{1} Y_{2}\right)$ holds. By a similar argument, $Y_{2} X_{2}$ is special and $R\left(Y_{2} X_{2}\right)=R\left(X_{1} X_{2}\right)$ holds. Thus, a special component $v\left(X_{2} Y_{2}\right)$ with $\left\langle v\left(X_{2} Y_{2}\right)\right\rangle=A_{Q}$ exists.

\section{ACKNOWLEDGMENTS}

We thank the referees for helpful comments. The first author's research was partially supported by JSPS KAKENHI Grant Numbers 25280004, 26330023, 17K00029. The second author's research was supported by JSPS Research Fellowship for Young Scientists. The third author's research was supported by The Mitsubishi Foundation, CREST, JST, Grant Number JPMJCR14D2, Japan, and JSPS KAKENHI Grant Number 26280004. The last author's research was supported by a Royal Society University Research Fellowship. This project has received funding from the European Research Council (ERC) under the European Union's Horizon 2020 research and innovation programme (grant agreement No 714532). The paper reflects only the authors' views and not the views of the ERC or the European Commission. The European Union is not liable for any use that may be made of the information contained therein.

\section{REFERENCES}

[1] U. Bertelé and F. Brioschi. Nonserial Dynamic Programming. Academic Press, 1972.

[2] E. Boros and P. L. Hammer. Pseudo-Boolean optimization. Discrete Applied Mathematics, 123:155-225, 2002.

[3] A. A. Bulatov. A dichotomy theorem for nonuniform CSPs. In Proceedings of the 58th Annual IEEE Symposium on Foundations of Computer Science (FOCS'17), pages 319-330, 2017.

[4] J. C. Colberson and P. Rudnicki. A fast algorithm for construction trees from distance matrices. Information Processing Letters, 30:215-220, 1989.

[5] M. C. Cooper and S. Živný. Hybrid tractability of valued constraint problems. Artificial Intelligence, 175:1555-1569, 2011.

[6] M. C. Cooper and S. Živný. Tractable triangles and cross-free convexity in discrete optimisation. fournal of Artificial Intelligence Research, 44:455-490, 2012.

[7] M. C. Cooper and S. Živný. Hybrid tractable classes of constraint problems. In A. Krokhin and S. Živný, editors, The Constraint Satisfaction Problem: Complexity and Approximability, volume 7 of Dagstuhl Follow-Ups Series, chapter 4, pages 113-135. Schloss Dagstuhl - Leibniz-Zentrum für Informatik, 2017.

[8] Y. Crama and P. L. Hammer. Boolean Functions-Theory, Algorithms, and Applications. Cambridge University Press, Cambridge, 2011.

[9] M. M. Deza and M. Laurent. Geometry of Cuts and Metrics. Springer, Heidelberg, 1997.

[10] A. W. M. Dress and W. Wenzel. Valuated matroids: A new look at the greedy algorithm. Applied Mathematics Letters, 3(2):33-35, 1990.

[11] A. W. M. Dress and W. Wenzel. Valuated matroids. Advances in Mathematics, 93:214-250, 1992. 
[12] J. Edmonds. Submodular functions, matroids, and certain polyhedra. In Gordon and Breach, editors, Combinatorial Structures and Their Applications, pages 69-87, New York, 1970.

[13] H. Hirai. A geometric study of the split decomposition. Discrete and Computational Geometry, 36:331-361, 2006.

[14] H. Hirai. Discrete convexity and polynomial solvability in minimum 0-extension problems. Mathematical Programming, Series A, 155:1-55, 2016.

[15] H. Hirai. L-convexity on graph structures. Journal of the Operations Research Society of Japan, 61:71-109, 2018.

[16] H. Hirai and Y. Iwamasa. Reconstructing phylogenetic tree from multipartite quartet system. In Proceedings of the 29th International Symposium on Algorithms and Computation (ISAAC'18), 2018.

[17] H. Hirai, Y. Iwamasa, K. Murota, and S. Živný. Beyond JWP: A tractable class of binary VCSPs via M-convex intersection. In Proceedings of the 35th International Symposium on Theoretical Aspects of Computer Science (STACS'18), volume 96 of Leibniz International Proceedings in Informatics, pages 39:1-39:14, 2018.

[18] H. Hirai and K. Murota. M-convex functions and tree metrics. Fapan fournal of Industrial and Applied Mathematics, 21:391-403, 2004.

[19] Y. Iwamasa. The quadratic M-convexity testing problem. Discrete Applied Mathematics, 238:106-114, 2018.

[20] Y. Iwamasa, K. Murota, and S. Živný. Discrete convexity in joint winner property. Discrete Optimization, 28:78-88, 2018.

[21] V. Kolmogorov, A. Krokhin, and M. Rolínek. The complexity of general-valued CSPs. SIAM fournal on Computing, 46(3):1087-1110, 2017.

[22] V. Kolmogorov, J. Thapper, and S. Živný. The power of linear programming for general-valued CSPs. SIAM fournal on Computing, 44(1):1-36, 2015.

[23] B. Korte and J. Vygen. Combinatorial Optimization: Theory and Algorithms. Springer, Heidelberg, 5th edition, 2010.

[24] A. Krokhin and S. Živný, editors. The Constraint Satisfaction Problem: Complexity and Approximability, volume 7 of Schloss Dagstuhl - Leibniz-Zentrum fuer Informatik. Dagstuhl Follow-Ups Series, 2017.

[25] K. Murota. Convexity and Steinitz's exchange property. Advances in Mathematics, 124:272-311, 1996.

[26] K. Murota. Valuated matroid intersection, I: optimality criteria. SIAM fournal on Discrete Mathematics, 9:545-561, 1996.

[27] K. Murota. Valuated matroid intersection, II: algorithms. SIAM fournal on Discrete Mathematics, 9:562-576, 1996.

[28] K. Murota. Discrete convex analysis. Mathematical Programming, 83:313-371, 1998.

[29] K. Murota. Matrices and Matroids for Systems Analysis. Springer, Heidelberg, 2000.

[30] K. Murota. Discrete Convex Analysis. SIAM, Philadelphia, 2003.

[31] K. Murota. Recent developments in discrete convex analysis. In W. Cook, L. Lovász, and J. Vygen, editors, Research Trends in Combinatorial Optimization, chapter 11, pages 219-260. Springer, Heidelberg, 2009.

[32] K. Murota. Discrete convex analysis: A tool for economics and game theory. fournal of Mechanism and Institution Design, 1(1):151-273, 2016.

[33] K. Murota and A. Shioura. Quadratic M-convex and L-convex functions. Advances in Applied Mathematics, 33:318-341, 2004.

[34] A. Schrijver. Combinatorial Optimization: Polyhedra and Efficiency. Springer, Heidelberg, 2003.

[35] C. Semple and M. Steel. Phylogenetics. Oxford University Press, Oxford, 2003.

[36] M. Steel. The complexity of reconstructing trees from qualitative characters and subtrees. Fournal of Classification, 9:91-116, 1992

[37] M. S. Waterman, T. F. Smith, M. Singh, and W. A. Beyer. Additive evolutionary trees. fournal of Theoretical Biology, 64:199-213, 1977.

[38] D. Zhuk. A proof of CSP dichotomy conjecture. In Proceedings of the 58th Annual IEEE Symposium on Foundations of Computer Science (FOCS'17), volume 331-342, 2017.

[39] S. Živný. The Complexity of Valued Constraint Satisfaction Problems. Springer, Heidelberg, 2012.

Received January 2018 